\title{
THE DEFOCUSING ENERGY-SUPERCRITICAL CUBIC NONLINEAR WAVE EQUATION IN DIMENSION FIVE
}

\author{
AYNUR BULUT
}

ABSTRACT. We consider the energy-supercritical nonlinear wave equation $u_{t t}$ $\Delta u+|u|^{2} u=0$ with defocusing cubic nonlinearity in dimension $d=5$ with no radial assumption on the initial data. We prove that a uniform-in-time a priori bound on the critical norm implies that solutions exist globally in time and scatter at infinity in both time directions. Together with our earlier works in dimensions $d \geq 6$ with general data and dimension $d=5$ with radial data, the present work completes the study of global well-posedness and scattering in the energy-supercritical regime for the cubic nonlinearity under the assumption of uniform-in-time control over the critical norm.

\section{Contents}

1. Introduction

2. Preliminaries

3. Improved integrability properties

4. Bounds of energy-flux type and subluminality

5. Improved decay properties

6. Proof of Theorem 1.1

\section{INTRODUCTION}

We consider the initial value problem for the nonlinear wave equation with defocusing cubic nonlinearity in the energy supercritical regime, that is, dimensions $d \geq 5$,

$$
\left\{\begin{aligned}
\partial_{t t} u-\Delta u+|u|^{2} u & =0, \\
\left.\left(u, \partial_{t} u\right)\right|_{t=0} & =\left(u_{0}, u_{1}\right) \in \dot{H}_{x}^{s_{c}} \times \dot{H}_{x}^{s_{c}-1},
\end{aligned}\right.
$$

where $u: I \times \mathbb{R}^{d} \rightarrow \mathbb{R}$ with $0 \in I \subset \mathbb{R}$ a time interval.

The scaling symmetry

$$
u(t, x) \mapsto u_{\lambda}(t, x):=\lambda u(\lambda t, \lambda x)
$$

maps the set of solutions of (IVP) to itself and, moreover, the $\dot{H}_{x}^{s_{c}} \times \dot{H}_{x}^{s_{c}-1}$ norm is preserved by the scaling, where we identify the critical regularity as $s_{c}=\frac{d-2}{2}$.

Received by the editors October 14, 2012 and, in revised form, December 23, 2012.

2010 Mathematics Subject Classification. Primary 35L71, 35B44, 35P25. 
We recall that solutions to (IVP) conserve the energy

$$
E\left(u(t), u_{t}(t)\right)=\int_{\mathbb{R}^{d}} \frac{1}{2}\left|u_{t}(t, x)\right|^{2}+\frac{1}{2}|\nabla u(t, x)|^{2}+\frac{1}{4}|u(t, x)|^{4} d x=E(u(0)),
$$

which is both finite and left invariant by the scaling when $s_{c}=1$. In this case, we refer to (IVP) as the energy-critical nonlinear wave equation. The range $s_{c}>1$, that is, $d \geq 5$, is therefore known as the energy-supercritical regime for NLW.

In a recent series of works [3, 4] treating dimensions $d \geq 6$ with general (possibly nonradial) initial data, and dimension $d=5$ with radial initial data, we proved that any solution to (IVP) which satisfies an a priori uniform-in-time bound over the critical norm must exist globally in time and scatter; see also [5] and [6]. The goal of the current paper is to treat the remaining case of dimension $d=5$ with non-radial initial data, completing the analysis of global well-posedness and scattering under the assumed a priori bound for (IVP) in the energy-supercritical regime.

More precisely, we consider strong solutions to

$$
\left\{\begin{aligned}
\partial_{t t} u-\Delta u+|u|^{2} u & =0, \\
\left.\left(u, \partial_{t} u\right)\right|_{t=0} & =\left(u_{0}, u_{1}\right) \in \dot{H}_{x}^{3 / 2} \times \dot{H}_{x}^{1 / 2},
\end{aligned}\right.
$$

that is, functions $u: I \times \mathbb{R}^{5} \rightarrow \mathbb{R}$ such that for every $K \subset I$ compact, $\left(u, u_{t}\right) \in$ $C_{t}\left(K ; \dot{H}_{x}^{3 / 2} \times \dot{H}_{x}^{1 / 2}\right)$ and $u \in L_{t, x}^{6}\left(K \times \mathbb{R}^{5}\right)$ which satisfy the Duhamel formula,

$$
u(t)=S(t)\left(u_{0}, u_{1}\right)+\int_{0}^{t} \frac{\sin \left(\left(t-t^{\prime}\right)|\nabla|\right)}{|\nabla|}\left|u\left(t^{\prime}\right)\right|^{2} u\left(t^{\prime}\right) d t^{\prime},
$$

where $S(t)\left(u_{0}, u_{1}\right)=\cos (t|\nabla|) u_{0}+\frac{\sin (t|\nabla|)}{|\nabla|} u_{1}$ is the solution to the linear wave equation with initial data $\left(u_{0}, u_{1}\right)$.

We refer to $I$ as the interval of existence of $u$, and we say that $I$ is the maximal interval of existence if $u$ cannot be extended to any larger time interval. We say that $u$ is a global solution if $I=\mathbb{R}$, and that $u$ is a blow-up solution if $\|u\|_{L_{t, x}^{6}\left(I \times \mathbb{R}^{d}\right)}=\infty$.

Our main result in this paper is the following theorem:

Theorem 1.1. Suppose that $u: I \times \mathbb{R}^{5} \rightarrow \mathbb{R}$ is a solution to (NLW) with maximal interval of existence $I \subset \mathbb{R}$ satisfying the a priori bound

$$
\left(u, u_{t}\right) \in L_{t}^{\infty}\left(I ; \dot{H}_{x}^{3 / 2} \times \dot{H}_{x}^{1 / 2}\right) .
$$

Then $I=\mathbb{R}$ and

$$
\|u\|_{L_{t, x}^{6}\left(\mathbb{R} \times \mathbb{R}^{5}\right)} \leq C
$$

for some constant $C=C\left(\left\|\left(u, u_{t}\right)\right\|_{L_{t}^{\infty}\left(\dot{H}_{x}^{3 / 2} \times \dot{H}_{x}^{1 / 2}\right)}\right)$. Furthermore, u scatters both forward and backward in time, i.e. there exist $\left(u_{0}^{ \pm}, u_{1}^{ \pm}\right) \in \dot{H}_{x}^{s_{c}} \times \dot{H}_{x}^{s_{c}-1}$ such that

$$
\left\|\left(u(t)-S(t)\left(u_{0}^{ \pm}, u_{1}^{ \pm}\right), u_{t}(t)-\partial_{t} S(t)\left(u_{0}^{ \pm}, u_{1}^{ \pm}\right)\right)\right\|_{\dot{H}_{x}^{s_{c}} \times \dot{H}_{x}^{s_{c}-1}} \rightarrow 0
$$

as $t \rightarrow \pm \infty$.

In the energy-critical case $s_{c}=1$ with the defocusing nonlinearity $|u|^{4 /(d-2)} u$, global well-posedness and scattering for the nonlinear wave equation was established in a number of works; see, for instance, [1, 10, 12,23, 26, 28, 30]. In particular, Struwe [29] established global well-posedness for radial inital data in dimension $d=3$, and Grillakis 10 removed this radial assumption. Grillakis then established global well-posedness and persistence of regularity in dimensions $3 \leq d \leq 5$ [11, while 
this result was obtained for all dimensions $d \geq 3$ by Shatah and Struwe [26-28] and Kapitanskii [12].

In all of these works in the energy-critical case, the key properties used are an immediate uniform-in-time control of the critical norm $\dot{H}_{x}^{1} \times L_{x}^{2}$ by virtue of the conservation of energy, along with the uniform space-time control given by the Morawetz estimate,

$$
\int_{I} \int_{\mathbb{R}^{d}} \frac{|u(t, x)|^{4}}{|x|} d x d t \lesssim E(u(0)) .
$$

Turning to the energy-supercritical regime $s_{c}>1$, the global well-posedness and scattering of solutions for large initial data remains an important open problem in this setting. In particular, the lack of any known conserved quantity at the critical regularity renders the problem significantly more difficult than the energy-critical case. Nevertheless, a number of recent works have treated the energy-supercritical nonlinear Schrödinger and nonlinear wave equations under an assumption of a uniform-in-time control of the critical norm, that is,

$$
\left(u, u_{t}\right) \in L_{t}^{\infty}\left(I ; \dot{H}_{x}^{s_{c}} \times \dot{H}_{x}^{s_{c}-1}\right),
$$

where $I \subset \mathbb{R}$ is the maximal interval of existence, in the spirit of the recent work of Escauriaza, Seregin and Sverak for Navier-Stokes [8].

The first such result was obtained by Kenig and Merle in [16] using the concentration compactness approach introduced in their study of the focusing energy-critical NLS and NLW [14,15]. In [16, global well-posedness and scattering was obtained for solutions satisfying (1.3) with radial initial data in dimension $d=3$ with the nonlinearity $|u|^{p} u$ with $p>4$, where the critical regularity becomes $s_{c}=\frac{d}{2}-\frac{2}{p}$; see also 17. Killip and Visan removed the radial assumption in dimension $d=321$. and established the result for radial initial data in dimensions $d \geq 3$ for a particular range of $p\left[22\right.$, which in dimension $d=5$ becomes $\frac{4}{3}<p<2$, disjoint from the cubic case treated in [4].

In 3 , we initiated the study of global well-posedness and scattering under the assumed a priori bound in the case of the cubic nonlinearity in dimensions $d \geq 6$ with no radial assumption on the initial data, and in 4 we extended this result to cover dimension $d=5$ with radial initial data. The role of the present work is therefore to complete the study of the defocusing cubic energy-supercritical NLW under the assumed uniform a priori control of the critical norm (1.3).

1.1. Outline of the proof of Theorem 1.1. Our proof of Theorem 1.1 makes use of the concentration-compactness approach of Kenig and Merle, and proceeds as follows:

Assuming that the theorem fails, the first step in the argument is to extract a minimal counterexample to the failure of global well-posedness and scattering; this counterexample will be referred to as a minimal blow-up solution. More precisely, we recall the following result:

Theorem 1.2 (Construction of a minimal blow-up solution, [15, 16]). Suppose that Theorem 1.1 failed. Then there exists a solution $u: I \times \mathbb{R}^{5} \rightarrow \mathbb{R}$ to (NLW) with maximal interval of existence $I$,

$$
\left(u, u_{t}\right) \in L_{t}^{\infty}\left(I ; \dot{H}_{x}^{3 / 2} \times \dot{H}_{x}^{1 / 2}\right), \quad \text { and } \quad\|u\|_{L_{t, x}^{6}\left(I \times \mathbb{R}^{5}\right)}=\infty,
$$


such that $u$ is a minimal blow-up solution in the following sense: for any solution $v$ with maximal interval of existence $J$ such that $\|v\|_{L_{t, x}^{6}\left(J \times \mathbb{R}^{5}\right)}=\infty$, we have

$$
\sup _{t \in I}\left\|\left(u(t), u_{t}(t)\right)\right\|_{\dot{H}_{x}^{3 / 2} \times \dot{H}_{x}^{1 / 2}} \leq \sup _{t \in J}\left\|\left(v(t), v_{t}(t)\right)\right\|_{\dot{H}_{x}^{3 / 2} \times \dot{H}_{x}^{1 / 2}} .
$$

Moreover, there exist $N: I \rightarrow \mathbb{R}^{+}$and $x: I \rightarrow \mathbb{R}^{5}$ such that the set

$$
K=\left\{\left(\frac{1}{N(t)} u\left(t, x(t)+\frac{x}{N(t)}\right), \frac{1}{N(t)^{2}} u_{t}\left(t, x(t)+\frac{x}{N(t)}\right)\right): t \in I\right\},
$$

has compact closure in $\dot{H}^{s_{c}} \times \dot{H}^{s_{c}-1}\left(\mathbb{R}^{5}\right)$.

The proof of Theorem 1.2 is based on concentration compactness ideas in the form of a profile decomposition result for the linear wave equation, established in dimension $d=3$ by Bahouri and Gerard in [1] and treated in dimensions $d \geq 3$ by the author in [2]. This profile decomposition states that, up to subsequences and the symmetries of the equation, any bounded sequence of initial data in the critical space $\dot{H}_{x}^{3 / 2} \times \dot{H}_{x}^{1 / 2}$ can be decomposed as a linear combination of pairwise orthogonal profiles and an error term which is small in a Strichartz norm. More precisely, we have

Proposition 1.1 (Profile decomposition [1,2]). Let $\left(u_{0, n}, u_{1, n}\right)_{n \in \mathbb{N}}$ be a bounded sequence in $\dot{H}_{x}^{3 / 2}\left(\mathbb{R}^{5}\right) \times \dot{H}_{x}^{1 / 2}\left(\mathbb{R}^{5}\right)$.

Then there exists a subsequence of $\left(u_{0, n}, u_{1, n}\right)$ (still denoted $\left(u_{0, n}, u_{1, n}\right)$ ), a sequence of profiles $\left(V_{0}^{j}, V_{1}^{j}\right)_{j \in \mathbb{N}} \subset \dot{H}_{x}^{3 / 2} \times \dot{H}_{x}^{1 / 2}$, and a sequence of triples $\left(\epsilon_{n}^{j}, x_{n}^{j}, t_{n}^{j}\right) \in$ $\mathbb{R}^{+} \times \mathbb{R}^{5} \times \mathbb{R}$, which are orthogonal in the sense that for every $j \neq j^{\prime}$,

$$
\frac{\epsilon_{n}^{j}}{\epsilon_{n}^{j^{\prime}}}+\frac{\epsilon_{n}^{j^{\prime}}}{\epsilon_{n}^{j}}+\frac{\left|t_{n}^{j}-t_{n}^{j^{\prime}}\right|}{\epsilon_{n}^{j}}+\frac{\left|x_{n}^{j}-x_{n}^{j^{\prime}}\right|}{\epsilon_{n}^{j}} \underset{n \rightarrow \infty}{\longrightarrow} \infty
$$

and for every $\ell \geq 1$, if

$$
V^{j}=S(t)\left(V_{0}^{j}, V_{1}^{j}\right) \quad \text { and } \quad V_{n}^{j}(t, x)=\frac{1}{\left(\epsilon_{n}^{j}\right)} V^{j}\left(\frac{t-t_{n}^{j}}{\epsilon_{n}^{j}}, \frac{x-x_{n}^{j}}{\epsilon_{n}^{j}}\right),
$$

then

$$
\left(u_{0, n}(x), u_{1, n}(x)\right)=\sum_{j=1}^{\ell}\left(V_{n}^{j}(0, x), \partial_{t} V_{n}^{j}(0, x)\right)+\left(w_{0, n}^{\ell}(x), w_{1, n}^{\ell}(x)\right)
$$

with

$$
\limsup _{n \rightarrow \infty}\left\|S(t)\left(w_{0, n}^{\ell}, w_{1, n}^{\ell}\right)\right\|_{L_{t}^{q} L_{x}^{r}} \underset{\ell \rightarrow \infty}{\longrightarrow} 0
$$

for every $(q, r)$ an $\dot{H}_{x}^{3 / 2}$-wave admissible pair with $q, r \in(2, \infty)$. For all $\ell \geq 1$, we also have,

$$
\begin{aligned}
& \left\|u_{0, n}\right\|_{\dot{H}_{x}^{3 / 2}}^{2}+\left\|u_{1, n}\right\|_{\dot{H}_{x}^{1 / 2}}^{2} \\
& \quad=\sum_{j=1}^{\ell}\left[\left\|V_{0}^{j}\right\|_{\dot{H}_{x}^{3 / 2}}^{2}+\left\|V_{1}^{j}\right\|_{\dot{H}_{x}^{1 / 2}}^{2}\right]+\left\|w_{0, n}^{\ell}\right\|_{\dot{H}_{x}^{3 / 2}}^{2}+\left\|w_{1, n}^{\ell}\right\|_{\dot{H}_{x}^{1 / 2}}^{2}+o(1), \quad n \rightarrow \infty .
\end{aligned}
$$

The next step in the argument consists of showing that such a minimal counterexample cannot occur. As we will see below, this failure of existence arises as a consequence of the compactness property (1.4). Before proceeding further, we now 
recall an equivalent formulation of (1.4) from [21,22] which will be an important tool in our analysis of blow-up solutions.

Definition 1.1 (Almost periodic modulo symmetries). A solution $u$ to (NLW) with time interval $I$ is said to be almost periodic modulo symmetries if $\left(u, u_{t}\right) \in$ $L_{t}^{\infty}\left(I ; \dot{H}_{x}^{s_{c}} \times \dot{H}_{x}^{s_{c}-1}\right)$ and there exist functions $N: I \rightarrow \mathbb{R}^{+}, x: I \rightarrow \mathbb{R}^{5}$ and $C: \mathbb{R}^{+} \rightarrow \mathbb{R}^{+}$such that for all $t \in I$ and $\eta>0$,

$$
\left.\left.\int_{|x-x(t)| \geq C(\eta) / N(t)}|| \nabla\right|^{s_{c}} u(t, x)\right|^{2}+\left.\left.|| \nabla\right|^{s_{c}-1} u_{t}(t, x)\right|^{2} d x \leq \eta
$$

and

$$
\left.\left.\int_{|\xi| \geq C(\eta) N(t)}|| \xi\right|^{3 / 2} \hat{u}(t, \xi)\right|^{2}+\left.\left.|| \xi\right|^{1 / 2} \hat{u}_{t}(t, \xi)\right|^{2} d \xi \leq \eta .
$$

Almost periodic solutions satisfy a reduced form of the Duhamel formula which states that the linear components of (1.1) vanish as the time $T$ approaches the endpoints of the maximal interval of existence $I$.

Proposition 1.2 (Duhamel formula for almost periodic solutions, 21, 32]). Let $u: I \times \mathbb{R}^{5} \rightarrow \mathbb{R}$ be a solution to (NLW) with maximal interval of existence $I$ which is almost periodic modulo symmetries. Then for all $t \in I$,

$$
\begin{gathered}
\left(\int_{t}^{T} \frac{\sin \left(\left(t-t^{\prime}\right)|\nabla|\right)}{|\nabla|} F\left(u\left(t^{\prime}\right)\right) d t^{\prime}, \int_{t}^{T} \cos \left(\left(t-t^{\prime}\right)|\nabla|\right) F\left(u\left(t^{\prime}\right)\right) d t^{\prime}\right) \\
\underset{T \rightarrow \sup I}{\rightarrow}\left(u(t), u_{t}(t)\right),
\end{gathered}
$$

and

$$
\begin{gathered}
\left(-\int_{T}^{t} \frac{\sin \left(\left(t-t^{\prime}\right)|\nabla|\right)}{|\nabla|} F\left(u\left(t^{\prime}\right)\right) d t^{\prime},-\int_{T}^{t} \cos \left(\left(t-t^{\prime}\right)|\nabla|\right) F\left(u\left(t^{\prime}\right)\right) d t^{\prime}\right) \\
\underset{T \rightarrow \inf I}{\longrightarrow}\left(u(t), u_{t}(t)\right),
\end{gathered}
$$

weakly in $\dot{H}_{x}^{3 / 2} \times \dot{H}_{x}^{1 / 2}$.

We now recall a refinement of Theorem 1.2, which states that any failure of Theorem 1.1 not only implies the existence of a minimal blow-up solution, but also implies that there exists an almost periodic solution which belongs to one of three particular blow-up scenarios, for which the function $N(t)$ is specified further.

Theorem 1.3 (Identification of blow-up scenarios, 21]). Suppose that Theorem 1.1 fails. Then there exists a solution $u: I \times \mathbb{R}^{5} \rightarrow \mathbb{R}$ to (NLW) with maximal interval of existence $I$ such that $u$ is almost periodic modulo symmetries,

$$
\left(u, u_{t}\right) \in L_{t}^{\infty}\left(\dot{H}_{x}^{3 / 2} \times \dot{H}_{x}^{1 / 2}\right), \quad \text { and } \quad\|u\|_{L_{t, x}^{6}\left(I \times \mathbb{R}^{5}\right)}=\infty,
$$

and $u$ satisfies one of the following:

- (finite time blow-up solution) either $\sup I<\infty$ or $\inf I>-\infty$.

- (soliton-like solution) $I=\mathbb{R}$ and $N(t)=1$ for all $t \in \mathbb{R}$.

- (low-to-high frequency cascade solution) $I=\mathbb{R}$ and

$$
\inf _{t \in \mathbb{R}} N(t) \geq 1, \quad \text { and } \quad \limsup _{t \rightarrow \infty} N(t)=\infty .
$$


To complete the proof of Theorem 1.1 it suffices to show that each of the scenarios identified in Theorem 1.3 cannot occur.

We begin with the finite time blow-up solution, which is ruled out in Proposition 6.1. The argument is based on the use of the finite speed of propagation and almost periodicity to show that up to a translation in space, the solution at a given time $t$ has compact support which shrinks to zero as $t$ approaches the blow-up time. The conservation of energy is then used to contradict the property that the solution is a blow-up solution. This argument is essentially the same as in higher dimensions (see [3] and the references cited therein); for completeness, we include a brief account of the proof in Section 6 .

We now turn to the remaining two scenarios, the soliton-like and low-to-high frequency cascade solutions. As in higher dimensions, the main tool that we use to rule out these scenarios is to show that due to their particular properties, the minimal blow-up solutions in these classes have finite energy. We then exploit the control given by the conseration of energy and the Morawetz estimate (1.2) to show that these solutions cannot exist.

Before proceeding with the current case of dimension five, let us first recall the main steps used to obtain the finiteness of energy for these two scenarios in dimensions six and higher:

- We first refine the bound $u \in L_{t}^{\infty} L_{x}^{d}$ (which is immediate from the Sobolev embedding and the a priori bound $\left.u \in L_{t}^{\infty} \dot{H}_{x}^{s_{c}}\right)$ to $L_{t}^{\infty} L_{x}^{p}$ for some $p<d$. In particular, we use a bootstrap argument to bound the low frequencies of $u$ via almost periodicity and the analogue of Proposition 1.2, while the high frequencies are bounded by the a priori bound.

- We next use this $L_{t}^{\infty} L_{x}^{p}$ bound to establish an implication of the form

$$
\left(u, u_{t}\right) \in L_{t}^{\infty}\left(\dot{H}_{x}^{s} \times \dot{H}_{x}^{s-1}\right) \quad \rightsquigarrow \quad\left(u, u_{t}\right) \in L_{t}^{\infty}\left(\dot{H}_{x}^{s-s_{0}} \times \dot{H}_{x}^{s-1-s_{0}}\right)
$$

for some $s_{0}>0$. This is accomplished by using the double Duhamel technique introduced in [7] and used in the context of the concentration compactness method in [19,20]. More precisely, we consider the homogeneous Sobolev norm as an inner product and use the Duhamel formula provided by Proposition 1.2 forward and backward in time on each side of the inner product, respectively. We remark that the resulting integrals are convergent in dimensions $d \geq 6$; this is the source of the dimensional restriction in our work 3 .

- Once we obtain the second step above, we iterate this argument starting with the a priori bound $\left(u, u_{t}\right) \in L_{t}^{\infty}\left(\dot{H}_{x}^{s_{c}} \times \dot{H}_{x}^{s_{c}-1}\right)$ to obtain the desired decay $L_{t}^{\infty}\left(\dot{H}_{x}^{1-\epsilon} \times \dot{H}_{x}^{-\epsilon}\right)$ for some $\epsilon>0$. In particular, we obtain that the energy is finite.

In the present work, in dimension $d=5$, we overcome the restriction in higher dimensions by using a covering argument to introduce a spatial localization into the Duhamel integrals. This is inspired by a similar approach used in 21] to study the energy-supercritical NLW in dimension $d=3$. However, substantial differences between the linear propagator, Strichartz and dispersive estimates in dimensions $d=3$ and $d=5$ require us to argue differently than in 21 .

We now outline the main tools involved in the present work as follows:

- A refined version of the improved $L_{t}^{\infty} L_{x}^{p}$ integrability obtained in dimensions $d \geq 6$. In particular, we establish the bound $u \in L_{t}^{\infty} L_{x}^{p}$ for some range 
of $p<5$; this shows that the a priori bound $\left(u, u_{t}\right) \in L_{t}^{\infty}\left(\dot{H}_{x}^{3 / 2} \times \dot{H}_{x}^{1 / 2}\right)$ implies that the soliton-like and low-to-high frequency cascade solutions belong to $L_{t}^{\infty} L_{x}^{3}$, and also enables us to exploit additional bounds of the form $\left(u, u_{t}\right) \in L_{t}^{\infty}\left(\dot{H}_{x}^{s} \times \dot{H}_{x}^{s-1}\right)$ to conclude the integrability $u \in L_{t}^{\infty} L_{x}^{p}$ for an expanded range of $p$ (see Proposition 3.1).

- A quantitative estimate on the decay of the $L_{t}^{\infty} L_{x}^{5}$ norm away from the centering function $x(t)$ (see Proposition 3.2). Such a result was first observed in 21] with the corresponding norm $L_{t}^{\infty} L_{x}^{3 p / 2}$ in dimension $d=3$. We remark that in our setting, Proposition 3.2 takes advantage of the improvement in $L_{t}^{\infty} L_{x}^{p}$ integrability given by Proposition 3.1 to give better decay when additional $L_{t}^{\infty}\left(\dot{H}_{x}^{s} \times \dot{H}_{x}^{s-1}\right)$ bounds are present. This improvement in decay is an essential aspect of our argument.

- A subluminality property which expresses that for a suitable class of solutions, the centering function $x(t)$ travels strictly slower than the propagation speed for (NLW). An analogous result was first obtained in [21] in the dimension $d=3$ setting. We note that the main estimate used to obtain the subluminality in [21] is the energy-flux inequality, while this estimate no longer provides the decay required in the current $d=5$ setting. To overcome this difficulty, we establish a frequency localized form of the energy-flux inequality which is accompanied by a frequency localized form of the concentration of potential energy (see Lemma 4.2 and Lemma 4.3).

With these tools in hand, we establish an iterative improvement in decay properties for the solution $u$. In particular, to make the Duhamel integrals convergent, we use a covering argument as in 21] to localize the resulting integrals in space, combined with a variant of a weak diffraction lemma from [21 adapted to the $d=5$ setting.

It is important to note that the applications of Proposition 3.1 and Proposition 3.2 in the iterative improvement of the decay in analogy to (1.5) use only the a priori bound $\left(u, u_{t}\right) \in L_{t}^{\infty}\left(\dot{H}_{x}^{3 / 2} \times \dot{H}_{x}^{1 / 2}\right)$ to obtain the decay $\left(u, u_{t}\right) \in L_{t}^{\infty}\left(\dot{H}_{x}^{s} \times \dot{H}_{x}^{s-1}\right)$ for $s>\frac{5}{4}$, which is still above the energy level. We then use this improved decay to obtain the integrability $L_{t}^{\infty} L_{x}^{p}$ for a larger range of $p$ than the one that is obtained via the a priori bound alone. This extra integrability in turn allows us to continue the iteration argument, and we conclude that the energy is finite.

We therefore again have access to the Morawetz estimate (1.2) and the conservation of energy. We then rule out the soliton-like and low-to-high frequency cascade scenarios, making use of almost periodicity, the finite speed of propagation, the Morawetz estimate (1.2) and the conservation of energy. This is accomplished in Proposition 6.2 and Proposition 6.3. respectively.

Outline of the paper. In Section 2, we establish our notation and recall some essential preliminaries concerning the wave equation, as well as some analytical tools which we will use in our arguments. In Section 3, we establish improved integrability properties of the soliton-like and low-to-high frequency cascade solutions. Then, in Section 4 we establish a frequency localized bound of energy-flux type and a frequency localized form of the concentration of potential energy, which are then used to prove the subluminality result. Finally, in Section 5 we prove that the global scenarios have finite energy, and in Section 6 we preclude each of the scenarios. 


\section{Preliminaries}

2.1. Notation. We will write $L_{t}^{q} L_{x}^{r}\left(I \times \mathbb{R}^{5}\right)$ to indicate the Banach space of functions $u: I \times \mathbb{R}^{5} \rightarrow \mathbb{R}$ with the space-time norm

$$
\|u\|_{L_{t}^{q} L_{x}^{r}}:=\left(\int_{I}\|u(t)\|_{L_{x}^{r}\left(\mathbb{R}^{5}\right)}^{q} d t\right)^{1 / q}<\infty
$$

with the standard convention when $q$ or $r$ is equal to infinity. If $q=r$, we shorten the notation and write $L_{t, x}^{q}$ in place of $L_{t}^{q} L_{x}^{q}$.

We write $X \lesssim Y$ to mean that there exists a constant $C>0$ such that $X \leq C Y$, while $X \lesssim u Y$ indicates that the constant $C=C_{u}$ may depend on $u$. We use the symbol $\nabla$ for the derivative operator in only the space variables.

Throughout the exposition, we define the Fourier transform on $\mathbb{R}^{5}$ by

$$
\widehat{f}(\xi)=(2 \pi)^{-5 / 2} \int_{\mathbb{R}^{5}} e^{-i x \cdot \xi} f(x) d x .
$$

We also denote the homogeneous Sobolev spaces by $\dot{H}_{x}^{s}\left(\mathbb{R}^{5}\right), s \in \mathbb{R}$, equipped with the norm

$$
\|f\|_{\dot{H}_{x}^{s}}=\left\||\nabla|^{s} f\right\|_{L_{x}^{2}},
$$

where the fractional differentiation operator is given by

$$
\widehat{|\nabla|^{s} f}(\xi)=|\xi|^{s} \widehat{f}(\xi) .
$$

We now recall the explicit formulation of the free propagator for the wave equation as

$$
S(t)(f, g)=\cos (t|\nabla|) f+\frac{\sin (t|\nabla|)}{|\nabla|} g,
$$

which is given in Fourier space by

$$
\widehat{S(t)}(f, g)=\cos (t|\xi|) \hat{f}(\xi)+\frac{\sin (t|\xi|)}{|\xi|} \hat{g}(\xi) .
$$

In dimension five, we have the additional representations

$$
\cos (t|\nabla|) f=\frac{3}{\left|S^{4}\right|} \partial_{t}\left[t^{-1} \partial_{t}\left(t^{-1} \int_{\partial B(x, t)} f(y) d S(y)\right)\right]
$$

and

$$
\frac{\sin (t|\nabla|)}{|\nabla|} g=\frac{3}{t\left|S^{4}\right|} \partial_{t}\left[\frac{1}{t} \int_{|y-x|=|t|} g(y) d S(y)\right],
$$

where $B(x, r)=\left\{x \in \mathbb{R}^{5}:|x|<r\right\}$ and $\left|S^{4}\right|$ denotes the surface area of the unit sphere $\partial B(0,1) \subset \mathbb{R}^{5}$.

We also recall the following expression of finite speed of propagation for almost periodic solutions, which we will frequently use in our arguments:

Lemma 2.1 (Speed of propagation for $x(t)$, 3, 21]). Suppose that $u: \mathbb{R} \times \mathbb{R}^{5} \rightarrow \mathbb{R}$ is an almost periodic solution to (NLW) with $N(t) \geq 1$ for all $t \in \mathbb{R}$. Then there exists $C>0$ such that for all $t, \tau \in \mathbb{R}$,

$$
|x(t)-x(\tau)| \leq|t-\tau|+C\left(N(t)^{-1}+N(\tau)^{-1}\right) .
$$


For a proof of this lemma, we refer the reader to (4.2) in [21] and, for a related argument in the case of the soliton-like solution, to [3. Lemma 8.2]. Although the result in [21] is stated for dimension $d=3$, the proof applies equally to the current setting.

2.2. Strichartz estimates for NLW. For $s \geq 0$, we say that a pair of exponents $(q, r)$ is $\dot{H}_{x}^{s}$-wave admissible if $q, r \geq 2, r<\infty$ and it satisfies

$$
\frac{1}{q}+\frac{2}{r} \leq 1, \quad \frac{1}{q}+\frac{5}{r}=\frac{5}{2}-s
$$

We also define the following Strichartz norms. For each $I \subset \mathbb{R}$ and $s \geq 0$, we set

$$
\begin{aligned}
\|u\|_{S_{s}(I)} & =\sup _{(q, r) \dot{H}_{x}^{s}-\text { wave admissible }}\|u\|_{L_{t}^{q} L_{x}^{r}\left(I \times \mathbb{R}^{5}\right)}, \\
\|u\|_{N_{s}(I)} & =\inf _{(q, r) \dot{H}_{x}^{s}-\text { wave admissible }}\|u\|_{L_{t}^{q^{\prime}} L_{x}^{r^{\prime}}\left(I \times \mathbb{R}^{5}\right)} .
\end{aligned}
$$

Suppose $u: I \times \mathbb{R}^{5} \rightarrow \mathbb{R}$ with time interval $0 \in I \subset \mathbb{R}$ is a solution to the nonlinear wave equation

$$
\left\{\begin{aligned}
u_{t t}-\Delta u+F & =0, \\
\left.\left(u, u_{t}\right)\right|_{t=0} & =\left(u_{0}, u_{1}\right) \in \dot{H}_{x}^{\mu} \times \dot{H}_{x}^{\mu-1}\left(\mathbb{R}^{5}\right), \quad \mu \in \mathbb{R} .
\end{aligned}\right.
$$

Then for all $s, \tilde{s} \in \mathbb{R}$ we have the inhomogeneous Strichartz estimates [9, 13,

$$
\begin{aligned}
\left\||\nabla|^{s} u\right\|_{S_{\mu-s}(I)}+\||\nabla|^{s-1} & u_{t} \|_{S_{\mu-s}(I)} \\
& \lesssim\left\|\left(u_{0}, u_{1}\right)\right\|_{\dot{H}_{x}^{\mu} \times \dot{H}_{x}^{\mu-1}}+\left\||\nabla|^{\tilde{s}} F\right\|_{N_{1+\tilde{s}-\mu}(I)} .
\end{aligned}
$$

We also recall the standard dispersive estimate for the linear propagator (see, for instance, [28]).

Lemma 2.2 (Dispersive estimate). For any $2 \leq p<\infty$ and $t \neq 0$, we have

$$
\left\|\frac{e^{i t|\nabla|}}{|\nabla|} f\right\|_{L_{x}^{p}} \lesssim|t|^{-2\left(1-\frac{2}{p}\right)}\left\||\nabla|^{2-\frac{6}{p}} f\right\|_{L_{x}^{p^{\prime}}}
$$

for all $f \in \mathcal{S}\left(\mathbb{R}^{5}\right)$, where $\frac{1}{p}+\frac{1}{p^{\prime}}=1$.

In particular,

$$
\left\|\frac{\sin (t|\nabla|)}{|\nabla|} f\right\|_{L_{x}^{p}} \lesssim|t|^{-2\left(1-\frac{2}{p}\right)}\left\||\nabla|^{2-\frac{6}{p}} f\right\|_{L_{x}^{p^{\prime}}}
$$

for all $f \in \mathcal{S}\left(\mathbb{R}^{5}\right)$.

2.3. Basic Littlewood-Paley theory. Let $\phi(\xi)$ be a real valued radially symmetric bump function supported in the ball $\left\{\xi \in \mathbb{R}^{5}:|\xi| \leq \frac{11}{10}\right\}$ which equals 1 on the ball $\left\{\xi \in \mathbb{R}^{5}:|\xi| \leq 1\right\}$. For any dyadic number $N=2^{k}, k \in \mathbb{Z}$, we define the following Littlewood-Paley operators:

$$
\begin{aligned}
\widehat{P_{\leq N} f}(\xi) & =\phi(\xi / N) \hat{f}(\xi), \\
\widehat{P_{>N} f}(\xi) & =(1-\phi(\xi / N) \hat{f}(\xi), \\
\widehat{P_{N} f}(\xi) & =(\phi(\xi / N)-\phi(2 \xi / N)) \hat{f}(\xi) .
\end{aligned}
$$

Similarly, we define $P_{<N}$ and $P_{\geq N}$ with

$$
P_{<N}=P_{\leq N}-P_{N}, \quad P_{\geq N}=P_{>N}+P_{N},
$$


and also

$$
P_{M<\cdot \leq N}:=P_{\leq N}-P_{\leq M}=\sum_{M<N_{1} \leq N} P_{N_{1}}
$$

whenever $M<N$.

These operators commute with one another and with derivative operators. Moreover, they are bounded on $L_{x}^{p}$ for $1 \leq p \leq \infty$ and obey the following Bernstein inequalities:

$$
\begin{aligned}
\left\||\nabla|^{s} P_{\leq N} f\right\|_{L_{x}^{p}} & \lesssim N^{s}\left\|P_{\leq N} f\right\|_{L_{x}^{p}}, \\
\left\|P_{>N} f\right\|_{L_{x}^{p}} & \lesssim N^{-s}\left\|P_{>N}|\nabla|^{s} f\right\|_{L_{x}^{p}}, \\
\left\||\nabla|^{ \pm s} P_{N} f\right\|_{L_{x}^{p}} & \sim N^{ \pm s}\left\|P_{N} f\right\|_{L_{x}^{p}}
\end{aligned}
$$

with $s \geq 0$ and $1 \leq p \leq \infty$.

In addition to the above Littlewood-Paley multiplier operators, we will also need the function $\theta: \mathbb{R}^{5} \rightarrow[0, \infty)$ defined by

$$
\theta(x)=\prod_{j=1}^{5}\left(\frac{\sin \left(x_{i}\right)}{x_{i}}\right)^{\alpha}, \quad \alpha>1,
$$

as well as the corresponding Fourier multiplier operator, given by

$$
\widehat{\theta(\nabla) f}=\theta(\xi) \hat{f}(\xi) .
$$

We observe that for all $x \in \mathbb{R}^{5}, 0 \leq \theta(x) \leq 1$, and that $\theta \in L^{1}$.

As mentioned in the introduction, the improvement of decay properties for $u$ in Section $[5$ is based on a localization procedure. Due to the nonlocal nature of the Littlewood-Paley and fractional derivative operators, as in 21 we will make use of the following lemma, which allows us to estimate the contributions far from the support of the localization.

Lemma 2.3 (Mismatch estimates, [21]). Let $\phi_{1}$ and $\phi_{2}$ be smooth functions on $\mathbb{R}^{5}$ such that $\max \left\{\left|\phi_{1}(x)\right|,\left|\phi_{2}(x)\right|\right\} \leq 1$ for all $x \in \mathbb{R}^{5}$ and such that there exists $A \geq 1$ with

$$
\operatorname{dist}\left(\operatorname{supp} \phi_{1}, \operatorname{supp} \phi_{2}\right) \geq A \text {. }
$$

Then for all $\sigma>0$ and $1 \leq p \leq q \leq \infty$ we have

$$
\left\|\phi_{1}|\nabla|^{\sigma} P_{\leq 1}\left(\phi_{2} f\right)\right\|_{L_{x}^{q}\left(\mathbb{R}^{5}\right)} \lesssim A^{-\sigma-\frac{5}{p}+\frac{5}{q}}\left\|\phi_{2} f\right\|_{L_{x}^{p}\left(\mathbb{R}^{5}\right)} .
$$

\section{IMPROVED INTEGRABILITY PROPERTIES}

In this section, we establish improved $L_{t}^{\infty} L_{x}^{p}$ integrability properties for a suitable class of almost periodic solutions to (NLW), which is the first step towards proving that the global solutions in the soliton-like and low-to-high frequency cascade scenarios have finite energy.

In particular, in Proposition 3.1 we prove that these solutions belong to the space $L_{t}^{\infty} L_{x}^{p}$ for some range of $p<5$, having recalled that they belong to $L_{t}^{\infty} L_{x}^{5}$ due to the a priori bound $\left(u, u_{t}\right) \in L_{t}^{\infty}\left(\dot{H}_{x}^{3 / 2} \times \dot{H}_{x}^{1 / 2}\right)$ combined with the Sobolev embedding. On the other hand, in Proposition 3.2 we establish an improved integrability property in a different direction, by obtaining a decay estimate on the $L_{t}^{\infty} L_{x}^{5}$ norm. 
3.1. Improved $L_{t}^{\infty} L_{x}^{p}$ integrability. We begin with Proposition 3.1, which provides $u \in L_{t}^{\infty} L_{x}^{p}$ integrability for some range of $p<5$. As mentioned in the introduction, an important aspect of our statement is the refinement of this estimate to provide improved $L_{t}^{\infty} L_{x}^{p}$ decay when additional knowledge of the decay properties of $u$ in the form $\left(u, u_{t}\right) \in L_{t}^{\infty}\left(\dot{H}_{x}^{s} \times \dot{H}_{x}^{s-1}\right), 1<s<\frac{3}{2}$, is present.

Proposition 3.1. Suppose that $u: \mathbb{R} \times \mathbb{R}^{5} \rightarrow \mathbb{R}$ is an almost periodic solution to (NLW) satisfying

$$
\left(u, u_{t}\right) \in L_{t}^{\infty}\left(\dot{H}_{x}^{s} \times \dot{H}_{x}^{s-1}\right) \cap L_{t}^{\infty}\left(\dot{H}_{x}^{3 / 2} \times \dot{H}_{x}^{1 / 2}\right)
$$

for some $s \in\left(1, \frac{3}{2}\right]$. Assume also that $\inf _{t \in \mathbb{R}} N(t) \geq 1$.

Then $u \in L_{t}^{\infty} L_{x}^{p}$ for every $p \in\left(\frac{10(3+2 s)}{15+4 s}, 5\right]$.

Before proceeding with the proof, we note that Proposition 3.1 in particular implies that for any global almost periodic solution $u$ to (NLW) satisfying the a priori bound

$$
\left(u, u_{t}\right) \in L_{t}^{\infty}\left(\mathbb{R} ; \dot{H}_{x}^{3 / 2} \times \dot{H}_{x}^{1 / 2}\right) \quad \text { with } \quad \inf _{t \in \mathbb{R}} N(t) \geq 1
$$

we have

$$
u \in L_{t}^{\infty} L_{x}^{3}
$$

Proof of Proposition 3.1. Our argument follows the broad outline established in the proof of Proposition 5.1 in [21] see also Lemma 7.2 in 3 . Fix $\eta>0$ to be determined later in the argument, and let $u$ be a solution to (NLW) as stated in Proposition 3.1. Since $u$ is an almost periodic solution with $N(t) \geq 1$ for all $t \in \mathbb{R}$, we may find a dyadic number $N_{0} \in(0,1)$ such that

$$
\left\||\nabla|^{3 / 2} u_{\leq N_{0}}\right\|_{L_{t}^{\infty} L_{x}^{2}}<\eta .
$$

As in 21, our proof of Proposition 3.1 will be based on a recurrence argument followed by an application of a discrete Gronwall inequality. Towards this end, we fix $r>5$ and define the quantity

$$
\mathcal{S}(N)=N^{(5-r) / r}\left\|u_{N}\right\|_{L_{t}^{\infty} L_{x}^{r}}
$$

We begin by noting that by Bernstein's inequalities followed by Sobolev's embedding and the a priori bound $u \in L_{t}^{\infty} \dot{H}_{x}^{3 / 2}$, we have

$$
\mathcal{S}(N) \lesssim N^{\frac{3}{2}}\left\|u_{N}\right\|_{L_{t}^{\infty} L_{x}^{2}} \lesssim\left\||\nabla|^{3 / 2} u_{N}\right\|_{L_{t}^{\infty} L_{x}^{2}}<\infty
$$

for $N>0$.

The following lemma then gives the necessary recurrence formula for the quantity $\mathcal{S}(N)$.

Lemma 3.1. For all dyadic numbers $N \leq 8 N_{0}$, we have

$$
\begin{gathered}
\mathcal{S}(N) \lesssim u\left(\frac{N}{N_{0}}\right)^{\frac{3}{2}}+\eta^{2} \sum_{\frac{2 N}{8} \leq N_{1} \leq N_{0}}\left(\frac{N}{N_{1}}\right)^{2} \mathcal{S}\left(N_{1}\right) \\
+\eta^{2} \sum_{N_{1} \leq \frac{N}{8}}\left(\frac{N_{1}}{N}\right)^{1 / 2} \mathcal{S}\left(N_{1}\right) .
\end{gathered}
$$


In particular, for every $\epsilon>0$ we have

$$
\mathcal{S}(N) \lesssim u, \epsilon N^{\frac{3}{2}-\epsilon}
$$

for all $N \leq 8 N_{0}$.

Proof. Fix $N \leq 8 N_{0}$. We first note that by the time translation symmetry, it suffices to prove the claim when $t=0$. Using the reduced form of the Duhamel formula given in Proposition 1.2 combined with Minkowski's inequality, we obtain

$$
\begin{aligned}
N^{\frac{5}{r}-1}\left\|u_{N}(0)\right\|_{L_{x}^{r}} \leq & N^{\frac{5}{r}-1}\left\|\int_{0}^{N^{-1}} \frac{\sin \left(-t^{\prime}|\nabla|\right)}{|\nabla|} P_{N} F\left(u\left(t^{\prime}\right)\right) d t^{\prime}\right\|_{L_{x}^{r}} \\
& +N^{\frac{5}{r}-1}\left\|\int_{N^{-1}}^{\infty} \frac{\sin \left(-t^{\prime}|\nabla|\right)}{|\nabla|} P_{N} F\left(u\left(t^{\prime}\right)\right) d t^{\prime}\right\|_{L_{x}^{r}} \\
= & :(I)+(I I) .
\end{aligned}
$$

To estimate $(I)$, we use the Bernstein inequality combined with the dispersive estimate (2.5) to get

$$
\begin{aligned}
(I) & \lesssim \int_{0}^{N^{-1}} N^{3 / 2}\left\|\frac{\sin \left(-t^{\prime}|\nabla|\right)}{|\nabla|} P_{N} F\left(u\left(t^{\prime}\right)\right)\right\|_{L_{x}^{2}} d t^{\prime} \\
& \lesssim \int_{0}^{N^{-1}} N^{3 / 2}\left\||\nabla|^{-1} P_{N} F\left(u\left(t^{\prime}\right)\right)\right\|_{L_{x}^{2}} d t^{\prime} \\
& \lesssim N\left\|P_{N} F(u(t))\right\|_{L_{t}^{\infty} L_{x}^{5 / 4}} .
\end{aligned}
$$

Similarly,

$$
\begin{aligned}
(I I) & \lesssim \int_{N^{-1}}^{\infty}\left\|\frac{\sin \left(-t^{\prime}|\nabla|\right)}{|\nabla|} P_{N} F\left(u\left(t^{\prime}\right)\right)\right\|_{L_{x}^{5}} d t^{\prime} \\
& \lesssim \int_{N^{-1}}^{\infty}|t|^{-6 / 5}\left\||\nabla|^{4 / 5} P_{N} F(u(t))\right\|_{L_{t}^{\infty} L_{x}^{5 / 4}} \\
& \lesssim N\left\|P_{N} F(u(t))\right\|_{L_{t}^{\infty} L_{x}^{5 / 4}}
\end{aligned}
$$

Collecting (3.5) and (3.6), we have

$$
N^{\frac{5}{r}-1}\left\|u_{N}(0)\right\|_{L_{x}^{r}} \lesssim u\left\|P_{N} F(u(t))\right\|_{L_{t}^{\infty} L_{x}^{5 / 4}} .
$$

We next estimate the right hand side of this inequality. Decomposing $u$ as

$$
u=u_{\leq N / 8}+u_{N / 8<\cdot \leq N_{0}}+u_{>N_{0}}=: u_{1}+u_{2}+u_{3},
$$

we write

$$
\begin{aligned}
\left\|P_{N} F(u)\right\|_{L_{t}^{\infty} L_{x}^{5 / 4}} \lesssim & \left\|P_{N}\left(u_{1}^{3}\right)\right\|_{L_{t}^{\infty} L_{x}^{5 / 4}}+\left\|P_{N}\left(u_{2}^{3}\right)\right\|_{L_{t}^{\infty} L_{x}^{5 / 4}} \\
& +\sum_{i, j=1}^{3}\left\|P_{N}\left(u_{3} u_{i} u_{j}\right)\right\|_{L_{t}^{\infty} L_{x}^{5 / 4}}+\sum_{i=1}^{2}\left\|P_{N}\left(u_{2} u_{1} u_{i}\right)\right\|_{L_{t}^{\infty} L_{x}^{5 / 4}} \\
= & :(I)+(I I)+\sum_{i, j=1}^{3}(I I I)_{i, j}+\sum_{i=1}^{2}(I V)_{i} .
\end{aligned}
$$

To establish (3.2), it now remains to estimate each of the terms $(I),(I I),(I I I)_{i, j}$ and $(I V)_{i}$ individually. 
We first remark that $(I)=0$, since the support of the Fourier transform of $u_{\leq N / 8}(t)^{3}$ gives that $P_{N}\left[u_{\leq N / 8}(t)^{3}\right] \equiv 0$.

To estimate the term $(\bar{I} I)$, we use Hölder's inequality along with the Sobolev embedding and Bernstein's inequality to obtain

$$
\begin{aligned}
(I I) & \lesssim N \sum_{2 N / 8 \leq N_{1} \leq N_{2} \leq N_{3} \leq N_{0}}\left\|u_{N_{1}} u_{N_{2}} u_{N_{3}}\right\|_{L_{t}^{\infty} L_{x}^{1}} \\
& \leq N \sum_{2 N / 8 \leq N_{1} \leq N_{2} \leq N_{3} \leq N_{0}}\left\|u_{N_{1}}\right\|_{L_{t}^{\infty} L_{x}^{r}}\left\|u_{N_{2}}\right\|_{L_{t}^{\infty} \frac{2 r}{L_{x}^{r-1}}}\left\|u_{N_{3}}\right\|_{L_{t}^{\infty} L_{x}^{\frac{2 r}{r-1}}} N_{1}^{(r-5) / r} \mathcal{S}\left(N_{1}\right)\left(N_{2} N_{3}\right)^{\frac{5}{2 r}-\frac{3}{2}}\left\|u_{\leq N_{0}}\right\|_{L_{t}^{\infty} \dot{H}_{x}^{3 / 2}}^{2} \\
& \lesssim N \sum_{2 N / 8 \leq N_{1} \leq N_{2} \leq N_{3} \leq N_{0}} \mathcal{S}\left(N_{1}\right) N_{1}^{-2} \\
& \lesssim{ } \eta^{2} N \sum_{2 N / 8 \leq N_{1} \leq N_{0}} \sum_{{ }_{2 N} \eta^{2} N^{-1} \sum_{\sum_{2} \leq N_{1} \leq N_{0}}\left(\frac{N}{N_{1}}\right)^{2} \mathcal{S}\left(N_{1}\right) .}
\end{aligned}
$$

Turning to the terms $(I I I)_{i, j}, i, j=1,2,3$, we note that for each such $i$ and $j$, Hölder's inequality followed by the Bernstein and Sobolev inequalities gives

$$
\begin{aligned}
(I I I)_{i, j} & \lesssim N^{1 / 2}\left\|u_{>N_{0}} u_{i} u_{j}\right\|_{L_{t}^{\infty} L_{x}^{10 / 9}} \\
& \leq N^{1 / 2}\left\|u_{>N_{0}}\right\|_{L_{t}^{\infty} L_{x}^{2}}\left\|u_{i}\right\|_{L_{t}^{\infty} L_{x}^{5}}\left\|u_{j}\right\|_{L_{t}^{\infty} L_{x}^{5}} \\
& \lesssim u N^{1 / 2} N_{0}^{-3 / 2}\|u\|_{L_{t}^{\infty} \dot{H}_{x}^{3 / 2}}^{3} \\
& \lesssim u N^{1 / 2} N_{0}^{-3 / 2} .
\end{aligned}
$$

We now estimate the terms $(I V)_{i}, i=1,2$, for which we examine each case separately. For the term $(I V)_{1}$, we write

$$
\begin{aligned}
(I V)_{1} & \lesssim\left\|u_{\frac{N}{8}<\cdot \leq N_{0}} u_{\leq \frac{N}{8}}^{2}\right\|_{L_{t}^{\infty} L_{x}^{5 / 4}}\left\|\sum_{N_{1} \leq N_{2} \leq \frac{N}{8}}\right\| u_{N_{1}}\left\|_{L_{t, x}^{\infty}}\right\| u_{N_{2}}\left\|_{L_{t}^{\infty} L_{x}^{10 / 3}}\right\|_{L_{t}^{\infty} L_{x}^{2}} N_{1}^{\frac{5}{r}}\left\|u_{N_{1}}\right\|_{L_{t}^{\infty} L_{x}^{r}}\left\|\nabla u_{N_{2}}\right\|_{L_{t}^{\infty} L_{x}^{2}} \\
& \lesssim{ }_{u} N^{-3 / 2} \eta \sum_{N_{1} \leq N_{2} \leq \frac{N}{8}} N_{1} \mathcal{S}\left(N_{1}\right) N_{2}^{-1 / 2} \\
& \lesssim{ }_{u} \eta^{2} N^{-3 / 2} \sum_{N_{1} \leq N_{2} \leq \frac{N}{8}} N_{N_{1} \leq \frac{N}{8}}\left(\frac{N_{1}}{N}\right)^{1 / 2} \mathcal{S}\left(N_{1}\right) .
\end{aligned}
$$


On the other hand, to estimate $(I V)_{2}$, we write

$$
\begin{aligned}
(I V)_{2} & \lesssim\left\|u_{\frac{N}{8}<\cdot \leq N_{0}}^{2} u_{\leq \frac{N}{8}}\right\|_{L_{t}^{\infty} L_{x}^{5 / 4}} \\
& \leq\left\|u_{\frac{N}{8}<\leq \leq N_{0}}\right\|_{L_{t}^{\infty} L_{x}^{2}}\left\|u_{\frac{N}{8}<\leq N_{0}}\right\|_{L_{t}^{\infty} L_{x}^{10 / 3}} \sum_{N_{1} \leq \frac{N}{8}}\left\|u_{N_{1}}\right\|_{L_{t, x}^{\infty}} \\
& \lesssim{ }_{u} \eta N^{-3 / 2}\left\|\nabla u_{\frac{N}{8}<\cdot \leq N_{0}}\right\|_{L_{t}^{\infty} L_{x}^{2}} \sum_{N_{1} \leq \frac{N}{8}} N_{1}^{\frac{5}{r}}\left\|u_{N_{1}}\right\|_{L_{t}^{\infty} L_{x}^{r}} \\
& \lesssim u \eta^{2} N^{-1} \sum_{N_{1} \leq \frac{N}{8}}\left(\frac{N_{1}}{N}\right) \mathcal{S}\left(N_{1}\right) .
\end{aligned}
$$

Putting the estimates (3.7)-(3.10) together, and observing that $N_{0} \leq 1$, we obtain (3.2) as desired.

To obtain (3.3), we first recall the following discrete version of the Gronwall inequality from [21]:

Lemma 3.2. Let $\gamma, \gamma^{\prime}, C, \eta>0$ and $\rho \in(0, \gamma)$ be given such that

$$
\eta \leq \frac{1}{4} \min \left\{1-2^{-\gamma}, 1-2^{-\gamma^{\prime}}, 1-2^{\rho-\gamma}\right\}
$$

Then for every bounded sequence $\left\{x_{k}\right\} \subset \mathbb{R}^{+}$satisfying

$$
x_{k} \leq C 2^{-\gamma k}+\eta \sum_{l=0}^{k-1} 2^{-\gamma(k-l)} x_{l}+\eta \sum_{l=k}^{\infty} 2^{-\gamma^{\prime}|k-l|} x_{l},
$$

we have $x_{k} \leq\left(4 C+\|x\|_{\ell^{\infty}}\right) 2^{-\rho k}$.

Fixing $0<\epsilon<\frac{3}{2}$ and using this lemma with $\gamma=\frac{3}{2}, \gamma^{\prime}=\frac{1}{2}, \rho<\gamma$ and $\eta>0$ sufficiently small, we obtain

$$
\mathcal{S}(N) \lesssim u, \epsilon N^{\frac{3}{2}-\epsilon}
$$

as desired (for a detailed description of a similar type of estimate see [21] and Appendix A of [3]). This completes the proof of Lemma 3.1.

Having obtained the recurrence formula in Lemma 3.1 we are now ready to complete the proof of Proposition 3.1. We begin by recalling that the bound $u \in$ $L_{t}^{\infty} L_{x}^{5}$ follows from the a priori bound $\left(u, u_{t}\right) \in L_{t}^{\infty}\left(\dot{H}_{x}^{3 / 2} \times \dot{H}_{x}^{1 / 2}\right)$ by the Sobolev embedding. 
Let $s \in\left(1, \frac{3}{2}\right]$ and $p \in\left(\frac{10(3+2 s)}{15+4 s}, \frac{4 s+10}{5}\right)$ be given. Then, using the LittlewoodPaley decomposition, Bernstein inequalities, and interpolation, we obtain

$$
\begin{aligned}
& \|u\|_{L_{t}^{\infty} L_{x}^{p}} \lesssim \sum_{N<8 N_{0}}\left\|u_{N}\right\|_{L_{t}^{\infty} L_{x}^{p}}+\sum_{N \geq 8 N_{0}} N^{1-\frac{5}{p}}\left\||\nabla|^{3 / 2} u_{N}\right\|_{L_{t}^{\infty} L_{x}^{2}} \\
& \lesssim u \sum_{N<8 N_{0}}\left\|u_{N}\right\|_{L_{t}^{\infty} L_{x}^{2}}^{\frac{2(r-p)}{p(r-2)}}\left\|u_{N}\right\|_{L_{t}^{\infty} L_{x}^{r}}^{\frac{r(p-2)}{p(r-2)}}+\sum_{N \geq 8 N_{0}} N^{1-\frac{5}{p}} \\
& \lesssim u \sum_{N<8 N_{0}}\left(N^{-s}\left\||\nabla|^{s} u_{N}\right\|_{L_{t}^{\infty} L_{x}^{2}}\right)^{\frac{2(r-p)}{p(r-2)}}\left\|u_{N}\right\|_{L_{t}^{\infty} L_{x}^{r}}^{\frac{r(p-2)}{p(r-2)}}+\sum_{N \geq 8 N_{0}} N^{1-\frac{5}{p}} \\
& \lesssim u 1+\sum_{N<8 N_{0}} N^{-\frac{2 s(r-p)}{p(r-2)}}\left(N^{1-\frac{5}{r}} \mathcal{S}(N)\right)^{\frac{r(p-2)}{p(r-2)}} \\
& \lesssim_{u} 1+\sum_{N<8 N_{0}} N^{-\frac{2 s(r-p)}{p(r-2)}}\left(N^{1-\frac{5}{r}+\left(\frac{3}{2}-\epsilon\right)}\right)^{\frac{r(p-2)}{p(r-2)}}
\end{aligned}
$$

for $r>5$ and $\epsilon>0$. Choosing $r=\frac{20 s+4 p s-35 p+70}{2(10+4 s-5 p)}, \epsilon=\frac{(10+4 s-5 p)(4 s p+15 p-30-20 s)}{4(p-2)(20 s+4 p s-35 p+70)}$, we obtain

$$
\|u\|_{L_{t}^{\infty} L_{x}^{p}} \lesssim u 1+\sum_{N<8 N_{0}} N^{\frac{(10+4 s-5 p)(4 s p+15 p-30-20 s)}{4 p(4 s-15 p+30+4 s p)}}<\infty
$$

where we observed that $p \in\left(\frac{10(3+2 s)}{15+4 s}, \frac{4 s+10}{5}\right)$ gives $\frac{(10+4 s-5 p)(4 s p+15 p-30-20 s)}{4 p(4 s-15 p+30+4 s p)}>0$. For the remaining values of $p$, the claim now follows by interpolating this bound with the bound $u \in L_{t}^{\infty} L_{x}^{5}$, which completes the proof of Proposition 3.1.

3.2. Quantitative $L_{t}^{\infty} L_{x}^{5}$ decay. We now establish a decay estimate for the $L_{t}^{\infty} L_{x}^{5}$ norm of global almost periodic solutions $u$ to (NLW) with inf $N(t) \geq 1$.

More precisely, we obtain the following proposition:

Proposition 3.2. Suppose $u: \mathbb{R} \times \mathbb{R}^{5} \rightarrow \mathbb{R}$ is an almost periodic solution to (NLW) with $\inf _{t \in \mathbb{R}} N(t) \geq 1$,

$$
\left(u, u_{t}\right) \in L_{t}^{\infty}\left(\dot{H}_{x}^{3 / 2} \times \dot{H}_{x}^{1 / 2}\right) \quad \text { and } \quad u \in L_{t}^{\infty} L_{x}^{w}
$$

for some $w \in(2,3]$. Then for every $\epsilon>0$ there exists $C_{u}>0$ such that for every $R \geq 1$,

$$
\sup _{t \in \mathbb{R}} \int_{|x-x(t)| \geq R}|u(t, x)|^{5} d x \leq C_{u} R^{-\frac{8(5-w)}{12-w}} .
$$

Our proof of Proposition 3.2 is based on rewriting the solution $u(t, x)$ using the Duhamel formula given by Proposition 1.2. Accordingly, we will need the following estimate for the long-time portion of the Duhamel formula.

Lemma 3.3. Suppose that $u: \mathbb{R} \times \mathbb{R}^{5} \rightarrow \mathbb{R}$ is an almost periodic solution to (NLW) with $\left(u, u_{t}\right) \in L_{t}^{\infty}\left(\dot{H}_{x}^{3 / 2} \times \dot{H}_{x}^{1 / 2}\right)$ and $\inf _{t \in \mathbb{R}} N(t) \geq 1$. Fix $4<p \leq 12$. Then there exists $C_{u}>0$ such that for every $0<S<T$ we have

$$
\left\|\int_{S}^{T} \frac{\sin (-t|\nabla|)}{|\nabla|} F(u(t)) d t\right\|_{L_{x}^{p}} \leq C_{u} S^{\frac{4}{p}-1} .
$$


Proof. Let $0<S<T$ be given. By an application of Minkowski's inequality followed by the dispersive estimate (2.5), we write

$$
\begin{aligned}
\left\|\int_{S}^{T} \frac{\sin (-t|\nabla|)}{|\nabla|} F(u(t)) d t\right\|_{L_{x}^{p}} & \lesssim \int_{S}^{T}\left\|\frac{\sin (-t|\nabla|)}{\nabla \mid} F(u(t))\right\|_{L_{x}^{p}} d t \\
& \lesssim \int_{S}^{T} t^{-2\left(1-\frac{2}{p}\right)}\left\||\nabla|^{2-\frac{6}{p}} F(u)\right\|_{L_{t}^{\infty} L_{x}^{p /(p-1)}} d t \\
& \lesssim \int_{S}^{T}(t)^{\frac{4}{p}-2} d t \\
& \lesssim S^{\frac{4}{p}-1}
\end{aligned}
$$

where we have used interpolation to obtain

$$
\begin{aligned}
\left\||\nabla|^{2-\frac{6}{p}} F(u)\right\|_{L_{t}^{\infty} L_{x}^{p /(p-1)}} & \lesssim\|u\|_{L_{t}^{\infty} L_{x}^{\frac{10 p}{2 p+1}}}^{2}\left\||\nabla|^{2-\frac{6}{p}} u\right\|_{L_{t}^{\infty} L_{x}^{\frac{5 p}{3(p-2)}}} \\
& \lesssim\|u\|_{L_{t}^{\infty} L_{x}^{3}}^{\frac{3}{2 p}}\|u\|_{L_{t}^{\infty} L_{x}^{5}}^{\frac{4 p-3}{2 p}}\|u\|_{L_{t}^{\infty} \dot{H}_{x}^{3 / 2}} \\
& \lesssim u 1,
\end{aligned}
$$

where we have noted that $u \in L_{t}^{\infty} L_{x}^{3}$ as a consequence of Proposition 3.1

With this lemma in hand, we are now ready to prove the quantitative decay result on the $L_{t}^{\infty} L_{x}^{5}$ norm, Proposition 3.2 .

Proof of Proposition 3.2. We argue as in the proof of Proposition 6.1 in [21], and begin by noting that by the space and time translation symmetries, we may assume without loss of generality that $t=0$ and $x(0)=0$. Let $\epsilon>0$ be given, fix $\delta>0$ to be determined, and define

$$
f(x):=\int_{\delta R}^{\infty} \frac{\sin \left(-t^{\prime}|\nabla|\right)}{|\nabla|} F\left(u\left(t^{\prime}\right)\right) d t^{\prime}, \quad g(x):=\int_{0}^{\delta R} \frac{\sin \left(-t^{\prime}|\nabla|\right)}{|\nabla|} F\left(u\left(t^{\prime}, x\right)\right) d t^{\prime} .
$$

Note that by using the no-waste Duhamel formula, followed by Hölder's inequality, Young's inequality and Lemma 3.3 with $p=12$, we obtain the bound

$$
\begin{aligned}
\int_{|x| \geq R}|u(0, x)|^{5} d x & \\
= & \int_{|x| \geq R}|f(x)+g(x)|^{\frac{12(5-w)}{12-w}}|u(0, x)|^{\frac{7 w}{12-w}} d x \\
\lesssim & \int_{|x| \geq R}|f(x)|^{\frac{12(5-w)}{12-w}}|u(0, x)|^{\frac{7 w}{12-w}}+|g(x)|^{\frac{12(5-w)}{12-w}}|u(0, x)|^{\frac{7 w}{12-w}} d x \\
\lesssim & \|f\|_{L_{x}^{12}}^{\frac{12(5-w)}{12-w}}\|u(0)\|_{L_{x}^{\frac{7 w}{12} w}}^{\frac{7 w}{12}} \\
& +\epsilon^{-\frac{5(12-w)}{12(5-w)}} \int_{|x| \geq R}|g(x)|^{5} d x+\epsilon^{\frac{5(12-w)}{7 w}} \int_{|x| \geq R}|u(0, x)|^{5} d x \\
\lesssim & (\delta R)^{-\frac{8(5-w)}{12-w}} \\
& +\epsilon^{-\frac{5(12-w)}{12(5-w)}} \int_{|x| \geq R}|g(x)|^{5} d x+\epsilon^{\frac{5(12-w)}{7 w}} \int_{|x| \geq R}|u(0, x)|^{5} d x
\end{aligned}
$$


for $\epsilon>0$. Choosing $\epsilon>0$ sufficiently small (depending on the implicit constant), this yields

$$
\int_{|x| \geq R}|u(0, x)|^{5} d x \lesssim u(\delta R)^{-\frac{8(5-w)}{12-w}}+\|g\|_{L_{x}^{5}(|x| \geq R)}^{5} .
$$

On the other hand, from the finite speed of propagation for the linear wave equation followed by the Sobolev embedding, we obtain

$$
\begin{aligned}
\|g\|_{L_{x}^{5}(|x| \geq R)} & \lesssim\left\|\int_{0}^{\delta R} \frac{\sin \left(-t^{\prime}|\nabla|\right)}{|\nabla|} F\left(u\left(t^{\prime}\right)\right) d t^{\prime}\right\|_{L_{x}^{5}(|x| \geq R)} \\
& \lesssim\left\|\int_{0}^{\delta R} \frac{\sin \left(t^{\prime}|\nabla|\right)}{|\nabla|}\left[F\left(u\left(t^{\prime}\right) \chi_{\Omega}(x)\right)\right] d t^{\prime}\right\|_{L_{x}^{5}} \\
& \lesssim\left\|\int_{0}^{\delta R} \frac{\sin \left(t^{\prime}|\nabla|\right)}{|\nabla|}\left[F\left(u\left(t^{\prime}\right) \chi_{\Omega}(x)\right)\right] d t^{\prime}\right\|_{\dot{H}_{x}^{3 / 2}}
\end{aligned}
$$

for any $\Omega \subset \mathbb{R}^{5}$ with $\{x:|x| \geq R-\delta R\} \subset \Omega$, where $\chi_{\Omega}$ is a smooth approximation to the characteristic function of $\Omega$.

We now recall that since $x(0)=0$, the bound (2.3) gives the existence of $C>0$ such that

$$
|x(t)| \leq|t|+C \leq \delta R+C
$$

for $t \in[0, \delta R]$. We may therefore take $\Omega=\{x:|x-x(t)| \geq R-2 \delta R-C\}$.

Substituting (3.14) into (3.13) and applying the inhomogeneous Strichartz estimate (backwards in time, applied to the solution of (NLW) with Cauchy data $(0,0)$ at $\left.t_{0}=\delta R\right)$, we obtain

$$
\begin{aligned}
(3.13) & \lesssim\left\||\nabla|^{5 / 4} F\left(u(t) \chi_{\Omega}(x)\right)\right\|_{L_{t}^{2}\left([0, \delta R] ; L_{x}^{4 / 3}\right)} \\
& \lesssim\left\|u(t) \chi_{\Omega}(x)\right\|_{L_{t}^{\infty}\left([0, \delta R] ; L_{x}^{5}\right)}\left\|u(t) \chi_{\Omega}(x)\right\|_{L_{t}^{2} L_{x}^{10}}\left\||\nabla|^{5 / 4} u(t) \chi_{\Omega}(x)\right\|_{L_{t}^{\infty} L_{x}^{20 / 9}} \\
& \lesssim B(R / 2)^{1 / 5}\left\|u(t) \chi_{\Omega}(x)\right\|_{L_{t}^{2} L_{x}^{10}}\left\|u(t) \chi_{\Omega}(x)\right\|_{L_{t}^{\infty} \dot{H}_{x}^{3 / 2}},
\end{aligned}
$$

provided that $R$ is sufficiently large and $\delta$ sufficiently small, where

$$
B(R):=\sup _{t \in \mathbb{R}} \int_{|x-x(t)| \geq R}|u(t, x)|^{5} d x .
$$

Fix $\eta>0$ to be determined. Using the finite speed of propagation, we may now choose $R_{0}=R_{0}(\eta, u)>0$ such that if $\Omega \subset\left\{x:|x| \geq R_{0}\right\}$, then

$$
\left\|u(t) \chi_{\Omega}\right\|_{L_{t}^{2} L_{x}^{10}}+\left\|u(t) \chi_{\Omega}\right\|_{L_{t}^{\infty} \dot{H}_{x}^{3 / 2}}<\eta
$$

which in conjunction with (3.15) gives

$$
\|g\|_{L_{x}^{5}(|x| \geq R)} \lesssim \eta^{2} B(R / 2)^{1 / 5}
$$

for $R \geq R_{0}$.

Combining (3.12) with (3.16), we obtain

$$
\int_{|x| \geq R}|u(0, x)|^{5} d x \lesssim u(\delta R)^{-\frac{8(5-w)}{12-w}}+\eta^{10} B(R / 2) .
$$


Applying the space and time translation symmetries,

$$
B(R) \leq C_{u}^{\prime}(\delta R)^{-\frac{8(5-w)}{12-w}}+\eta^{10} C_{u}^{\prime} B(R / 2)
$$

for each $\eta>0$ and every $R \geq R_{0}(\eta, u)$.

On the other hand, by the a priori bound $\left(u, u_{t}\right) \in L_{t}^{\infty}\left(\dot{H}_{x}^{3 / 2} \times \dot{H}_{x}^{1 / 2}\right)$ we have $B(R) \lesssim 1$ for all $R<R_{0}(\eta, u)$. Invoking an induction argument and choosing $\eta>0$ sufficiently small (to prevent the constants from blowing up in the induction), we obtain

$$
B(R) \leq C_{u} R^{-\frac{8(5-w)}{12-w}}
$$

for all $R$ as desired.

\section{Bounds OF ENERGY-FLUX TYPE AND SUBLUMINALITY}

In this section, our main goal is to establish an improvement upon the classical finite speed of propagation for (NLW) known as subluminality, which states that for the soliton-like and low-to-high frequency cascade solutions, the centering function $x(t)$ in the definition of almost periodicity travels strictly slower than the propagation speed of the equation; this is the content of Theorem 4.1. The main tools we will use to establish this property are a frequency localized form of the energy-flux bound, which will be established in Lemma 4.2, and a frequency localized version of the concentration of potential energy, Lemma 4.3 .

We begin by recalling a form of the standard energy flux bound in the $\mathbb{R}^{5}$ setting (see, for instance [21, 31]).

Lemma 4.1 (Energy-flux inequality). For every solution $u$ to $(\mathrm{NLW})$ with $\left(u, u_{t}\right) \in$ $L_{t}^{\infty}\left(I ; \dot{H}_{x}^{3 / 2} \times \dot{H}_{x}^{1 / 2}\right)$, there exists $C_{u}>0$ such that we have

$$
\int_{I_{0}} \int_{|x-y|=|t|}|u(t, x)|^{4} d S(x) d t \leq C_{u} \sup _{t \in I_{0}}|t|
$$

for every $I_{0} \subset I$ and $y \in \mathbb{R}^{5}$.

The subluminality property obtained in 21] makes essential use of the fact that the right hand side of the energy-flux inequality grows sublinearly in $|t|$ in dimension $d=3$, which is no longer the case in our setting. To overcome this, we localize the estimate in frequency.

In the frequency localized setting, the analogue of the energy flux bound must take into account the fact that $u_{\geq N}$ satisfies

$$
\partial_{t t} u_{\geq N}-\Delta u_{\geq N}+P_{\geq N}\left[u^{3}\right]=0,
$$

in place of (NLW). In view of this, our frequency localized form of the energy-flux bound is the following:

Lemma 4.2 (Frequency localized energy-flux bound). Suppose that $u: \mathbb{R} \times \mathbb{R}^{5} \rightarrow \mathbb{R}$ is an almost periodic solution to (NLW) with $\left(u, u_{t}\right) \in L_{t}^{\infty}\left(\mathbb{R} ; \dot{H}_{x}^{3 / 2} \times \dot{H}_{x}^{1 / 2}\right)$ and $\inf N(t) \geq 1$. Then there exists $C_{u}>0$ such that for every $\eta \in(0,1)$ there exists $N_{0}=N_{0}(\eta)$ such that for every dyadic $N<N_{0}, x \in \mathbb{R}^{5}$ and compact $I_{0} \subset \mathbb{R}$, we have

$$
\int_{I_{0}} \int_{|x-y|=|t|}\left|u_{\geq N}(t, y)\right|^{4} d S(y) d t \leq C_{u}\left(N^{-1}+\eta\left|I_{0}\right|\right) .
$$


Proof. In the following argument, we assume that $u$ is smooth with compact support (so that integration by parts is justified); we note that this assumption can be removed by standard approximation arguments. We work on the interval $I_{0}^{+}=$ $I_{0} \cap[0, \infty)$ and note that the proof for $I_{0} \cap(-\infty, 0]$ is similar. Fix $N>0$ and define

$$
\mathcal{E}(t)=\int_{|x-y| \leq t} \frac{1}{2}\left|\partial_{t} u_{\geq N}(t, x)\right|^{2}+\frac{1}{2}\left|\nabla u_{\geq N}(t, x)\right|^{2}+\frac{1}{4}\left|u_{\geq N}(t, x)\right|^{4} d x
$$

for $t \in I_{0}^{+}$. Differentiation in time followed by integration by parts gives

$$
\begin{aligned}
& \frac{d \mathcal{E}}{d t}(t)=\int_{|x-y|=t}\left[\frac{1}{2}\left|\partial_{t} u_{\geq N}(t, x)\right|^{2}+\frac{1}{2}\left|\nabla u_{\geq N}(t, x)\right|^{2}+\frac{1}{4}\left|u_{\geq N}(t, x)\right|^{4}\right. \\
& \left.\quad+\nabla u_{\geq N}(t, x) \partial_{t} u_{\geq N}(t, x) \cdot \frac{x-y}{|x-y|}\right] d S(x) \\
& +\int_{|x-y| \leq t}\left[\partial_{t} u_{\geq N}(t, x) \partial_{t t} u_{\geq N}(t, x)+\left[-\Delta u_{\geq N}(t, x)\right] \partial_{t} u_{\geq N}(t, x)\right. \\
& \left.+u_{\geq N}(t, x)^{3} \partial_{t} u_{\geq N}(t, x)\right] d x .
\end{aligned}
$$

Using (4.2) we get

$$
\begin{aligned}
\frac{d \mathcal{E}}{d t}(t)= & \int_{|x-y|=t}\left[\frac{1}{2}\left|\partial_{t} u_{\geq N}(t, x)\right|^{2}+\frac{1}{2}\left|\nabla u_{\geq N}(t, x)\right|^{2}+\frac{1}{4}\left|u_{\geq N}(t, x)\right|^{4}\right. \\
& \left.+\nabla u_{\geq N}(t, x) \partial_{t} u_{\geq N}(t, x) \cdot \frac{x-y}{|x-y|}\right] d S(x) \\
& +\int_{|x-y| \leq t}\left[\left(u_{\geq N}(t, x)^{3}-P_{\geq N}\left[u(t, x)^{3}\right]\right) \partial_{t} u_{\geq N}(t, x)\right] d x \\
\geq \frac{1}{4} \int_{|x-y|=t}\left|u_{\geq N}(t, x)\right|^{4} d x & \quad-\left|\int_{|x-y| \leq t}\left[\left(u_{\geq N}(t, x)^{3}-P_{\geq N}\left[u(t, x)^{3}\right]\right) \partial_{t} u_{\geq N}(t, x)\right] d x\right| .
\end{aligned}
$$

Our next goal is to establish the bound

$$
\left\|\left(u_{\geq N}(t, x)^{3}-P_{\geq N}\left[u(t, x)^{3}\right]\right) \partial_{t} u_{\geq N}(t, x)\right\|_{L_{t}^{\infty} L_{x}^{1}} \lesssim u \eta
$$

on the second term in (4.3), for $N$ sufficiently small.

To obtain (4.4), note that using Hölder's inequality and the Sobolev embedding gives

$$
\begin{aligned}
& \left\|\left(u_{\geq N}(t, x)^{3}-P_{\geq N}\left[u(t, x)^{3}\right]\right) \partial_{t} u_{\geq N}(t, x)\right\|_{L_{t}^{\infty} L_{x}^{1}} \\
& \lesssim\left\|u_{\geq N}(t, x)^{3}-P_{\geq N}\left[u(t, x)^{3}\right]\right\|_{L_{t}^{\infty} L_{x}^{5 / 3}}\left\|\partial_{t} u_{\geq N}(t, x)\right\|_{L_{t}^{\infty} \dot{H}_{x}^{1 / 2}} \\
& \lesssim u\left\|u_{\geq N}(t, x)^{3}-P_{\geq N}\left[u_{\geq N}^{3}\right]\right\|_{L_{t}^{\infty} L_{x}^{5 / 3}}+\left\|u_{<N}\right\|_{L_{x}^{5}}\|u\|_{L_{t}^{\infty} L_{x}^{5}}^{2},
\end{aligned}
$$

where we have used the decomposition $u=u_{<N}+u_{\geq N}$.

Now, using the almost periodicity and $\inf N(t) \geq 1$, we may choose $N$ and $N_{1}$ small enough so that

$$
N<\eta^{3 / 2} N_{1} \quad \text { and } \quad\left\|\left(u_{\leq N_{1}}, \partial_{t} u_{\leq N_{1}}\right)\right\|_{L_{t}^{\infty}\left(\dot{H}_{x}^{3 / 2} \times \dot{H}_{x}^{1 / 2}\right)}<\eta
$$


are satisfied. Then, writing $u_{\geq N}^{3}-P_{\geq N}\left[u_{\geq N}^{3}\right]$ as $P_{<N}\left[u_{\geq N}^{3}\right]$, we use the Bernstein and Hölder inequalities and the decomposition $u_{\geq N}=u_{N \leq \cdot<N_{1}}+u_{\geq N_{1}}$, followed by another instance of Bernstein's inequality and the Sobolev embedding to obtain

$$
\begin{aligned}
\text { (4.5) } & \lesssim_{u}\left\|P_{<N}\left[u_{\geq N}^{3}\right]\right\|_{L_{t}^{\infty} L_{x}^{5 / 3}}+\eta \\
& \lesssim_{u} N^{2 / 3}\left\|u_{\geq N}^{3}\right\|_{L_{t}^{\infty} L_{x}^{15 / 11}}+\eta \\
& \lesssim_{u} N^{2 / 3}\left\|u_{\geq N}\right\|_{L_{t}^{\infty} L_{x}^{5}}^{2}\left\|u_{\geq N}\right\|_{L_{t}^{\infty} L_{x}^{3}}+\eta \\
& \lesssim_{u} N^{2 / 3}\left(\left\|u_{N \leq \cdot<N_{1}}\right\|_{L_{t}^{\infty} L_{x}^{3}}+\left\|u_{\geq N_{1}}\right\|_{L_{t}^{\infty} L_{x}^{3}}\right)+\eta \\
& \lesssim_{u} N^{2 / 3}\left(N^{-2 / 3}\left\||\nabla|^{2 / 3} u_{N \leq \cdot<N_{1}}\right\|_{L_{t}^{\infty} L_{x}^{3}}+N_{1}^{-2 / 3}\left\||\nabla|^{2 / 3} u_{\geq N_{1}}\right\|_{L_{t}^{\infty} L_{x}^{3}}\right)+\eta \\
& \lesssim_{u}\left\|u_{N \leq \cdot<N_{1}}\right\|_{L_{t}^{\infty} \dot{H}_{x}^{3 / 2}}+\left(\frac{N}{N_{1}}\right)^{2 / 3}\left\|u_{\geq N_{1}}\right\|_{L_{t}^{\infty} \dot{H}_{x}^{3 / 2}}+\eta \\
& \lesssim_{u} \eta .
\end{aligned}
$$

This completes the proof of the inequality (4.4).

Combining (4.3) with (4.4), integrating over $t \in I_{0}$, and invoking the Fundamental Theorem of Calculus, we obtain

$$
\begin{aligned}
& \int_{I_{0}^{+}} \int_{|x-y|=t}\left|u_{\geq N}(t, x)\right|^{4} d x d t \\
& \quad \lesssim \sup _{t \in I_{0}}|\mathcal{E}(t)|+\int_{I_{0}^{+}} \int_{|x-y| \leq|t|}\left|\left(u_{\geq N}(t, x)^{3}-P_{\geq N}\left[u(t, x)^{3}\right]\right) \partial_{t} u_{\geq N}(t, x)\right| d x d t \\
& \quad \lesssim \sup _{t \in I_{0}}|\mathcal{E}(t)|+\eta\left|I_{0}^{+}\right|,
\end{aligned}
$$

provided that $N$ is sufficiently small. To bound the right hand side, we use the Bernstein inequalities, followed by Hölder's inequality and the Sobolev embedding to obtain

$$
\begin{aligned}
\mathcal{E}(t) & \lesssim N^{-1}\left\|\left(u, \partial_{t} u\right)\right\|_{L_{t}^{\infty}\left(\dot{H}_{x}^{3 / 2} \times \dot{H}_{x}^{1 / 2}\right)}^{2}+N^{-1}\left\|P_{\geq N}\left[u(t, x)^{3}\right]\right\|_{L_{x}^{5 / 3}}\left\|\nabla u_{\geq N}\right\|_{L_{x}^{5 / 2}} \\
& \lesssim u N^{-1}
\end{aligned}
$$

for every $t \in I_{0}$, which gives the desired estimate, completing the proof of Lemma 4.2 .

In order to invoke the frequency localized energy flux bound, Lemma 4.2, in the argument for subluminality, we must adapt the bound on concentration of potential energy to account for the frequency localization.

Towards this end, in the remainder of this section, we let $u: \mathbb{R} \times \mathbb{R}^{5} \rightarrow \mathbb{R}$ be an almost periodic solution to (NLW) satisfying $\left(u, u_{t}\right) \in L_{t}^{\infty}\left(\mathbb{R} ; \dot{H}_{x}^{3 / 2} \times \dot{H}_{x}^{1 / 2}\right)$ such that $\inf N(t) \geq 1$.

Recall that (see, e.g. [21, 3]) there exists $C_{u}>0$ such that for every $k \geq 1$,

$$
\int_{I_{0}} \int_{|x-x(t)| \leq C / N(t)}|u(t, x)|^{4} d x d t \geq C_{u} \int_{I_{0}} N(t)^{-1} d t .
$$

As we noted earlier, we will need a frequency localized form of this bound. More precisely,

Lemma 4.3 (Frequency localized potential energy concentration). Let $u: \mathbb{R} \times \mathbb{R}^{5} \rightarrow$ $\mathbb{R}$ be an almost periodic solution to (NLW) satisfying $\left(u, u_{t}\right) \in L_{t}^{\infty}\left(\mathbb{R} ; \dot{H}_{x}^{3 / 2} \times \dot{H}_{x}^{1 / 2}\right)$ 
such that $\inf N(t) \geq 1$. Then there exists $N_{0}>0$ and $C_{u}>0$ such that for all $N \leq N_{0}$ and $k \geq 1$,

$$
\int_{I_{0}} \int_{|x-x(t)| \leq C / N(t)}\left|u_{\geq 8 N}(t, x)\right|^{4} d x d t \geq C_{u} \int_{I_{0}} N(t)^{-1} d t .
$$

Before proceeding with the proof, we recall a consequence of almost periodicity which will aid in estimating the error terms resulting from the frequency localization of the potential energy.

More precisely, let $u$ be a solution given as in Lemma 4.3. Then, by the definition of almost periodicity along with the property $\inf _{t \in[0, \infty)} N(t) \geq 1$, we have

$$
\lim _{N \rightarrow 0}\left[\left\|u_{\leq N}\right\|_{L_{t}^{\infty} \dot{H}_{x}^{3 / 2}}+\left\|P_{\leq N} u_{t}\right\|_{L_{t}^{\infty} \dot{H}_{x}^{1 / 2}}\right]=0 .
$$

Proof of Lemma 4.3. Fix $N>0$. Applying (4.6) to $u_{\geq 8 N}$, we obtain

$$
\begin{aligned}
& {\left[\int_{I_{0}} \int_{|x-x(t)| \leq C / N(t)}\left|u_{\geq 8 N}(t, x)\right|^{4} d x d t\right]^{1 / 4}} \\
& \geq\left[\int_{I_{0}} \int_{|x-x(t)| \leq C / N(t)}|u(t, x)|^{4} d x d t\right]^{1 / 4} \\
& \quad-\left[\int_{I_{0}} \int_{|x-x(t)| \leq C / N(t)}\left|u_{<8 N}(t, x)\right|^{4} d x d t\right]^{1 / 4} \\
& \gtrsim_{u} C_{u} \int_{I_{0}} N(t)^{-1} d t-\left[\int_{I_{0}} \int_{|x-x(t)| \leq C / N(t)}\left|u_{<8 N}(t, x)\right|^{4} d x d t\right]^{1 / 4} .
\end{aligned}
$$

It therefore suffices to estimate the space-time norm appearing in (4.9). For this, we fix $\eta \in(0,1)$ to be determined later in the argument, and use Hölder's inequality followed by the decomposition $u=u_{<8 N}+u_{\geq 8 N}$ to obtain

$$
\begin{aligned}
\int_{I_{0}} \int_{|x-x(t)| \leq C / N(t)}\left|u_{<8 N}(t, x)\right|^{4} d x d t & \leq \int_{I_{0}} \frac{C}{N(t)}\left\|u_{<8 N}(t, x)\right\|_{L_{x}^{5}}^{4} d t \\
& \lesssim u \eta \int_{I_{0}} N(t)^{-1} d t,
\end{aligned}
$$

where we have used (4.8) to obtain the second inequality.

Substituting (4.10) into (4.9), we then have

$$
\int_{I_{0}} \int_{|x-x(t)| \leq C / N(t)}\left|u_{\geq 8 N}(t, x)\right|^{4} d x d t \geq\left(C_{u}-\eta C_{u}^{\prime}\right) \int_{I_{0}} N(t)^{-1} d t .
$$

Choosing $\eta$ small enough so that $\eta C_{u}^{\prime}<C_{u} / 2$ yields the bound (4.7).

Having obtained the frequency localized energy flux bound and potential energy concentration we now turn to the proof of the subluminality property. For this purpose, we recall the following lemma from [21, which states that the function $x(t)$ can be chosen to have a certain centering property and establishes a first relationship between the speed of $x(t)$ and the frequency scale function $N(t)$.

Lemma 4.4 ([21]). Suppose that $u: \mathbb{R} \times \mathbb{R}^{5} \rightarrow \mathbb{R}$ is an almost periodic solution to $(\mathrm{NLW})$ satisfying $\left(u, u_{t}\right) \in L_{t}^{\infty}\left(\mathbb{R} ; \dot{H}_{x}^{3 / 2} \times \dot{H}_{x}^{1 / 2}\right)$ such that $\inf N(t) \geq 1$. 
Then there exists a constant $C_{u}>0$ such that $x(t)$ can be chosen to satisfy

$$
\left.\left.\inf _{\omega \in S^{4}} \int_{\omega \cdot(x-x(t))>0}|| \nabla\right|^{3 / 2} u(t, x)\right|^{2}+\left.\left.|| \nabla\right|^{1 / 2} u_{t}(t, x)\right|^{2} d x \geq \frac{1}{C_{u}} .
$$

Moreover, there exists $c=c_{u} \in(0,1)$ such that for all $\tau_{1}, \tau_{2} \in \mathbb{R}$ satisfying $N\left(\tau_{1}\right) \leq N\left(\tau_{2}\right)$, we have

$$
\left|x\left(\tau_{1}\right)-x\left(\tau_{2}\right)\right| \geq\left|\tau_{1}-\tau_{2}\right|-c N\left(\tau_{1}\right)^{-1} \quad \Rightarrow \quad N\left(\tau_{1}\right) \geq c^{2} N\left(\tau_{2}\right) .
$$

The arguments used to obtain the claims in Lemma 4.4 follow from the small data theory and the finite speed of propagation; in particular, the discussion of Proposition 4.1 and Lemma 4.4 in [21] applies equally well to the current setting.

We are now ready to prove the main result of this section,

Theorem 4.1 (Subluminality). Suppose that $u: \mathbb{R} \times \mathbb{R}^{5} \rightarrow \mathbb{R}$ is an almost periodic solution to (NLW) satisfying $\left(u, u_{t}\right) \in L_{t}^{\infty}\left(\dot{H}_{x}^{3 / 2} \times \dot{H}_{x}^{1 / 2}\right)$ and such that $\inf _{t \in \mathbb{R}} N(t) \geq$ 1. Then there exists $\delta>0$ such that for every $t, \tau \in \mathbb{R}$ with $|t-\tau| \geq \delta^{-1}$, we have

$$
|x(t)-x(\tau)| \leq(1-\delta)|t-\tau| .
$$

Proof. As in 21, we note that it suffices to show the following claim: there exists $A>1$ such that for every $t_{0} \in \mathbb{R}$ there exists $t \in\left[t_{0}, t_{0}+A N\left(t_{0}\right)^{-1}\right]$ such that

$$
\left|x(t)-x\left(t_{0}\right)\right| \leq\left|t-t_{0}\right|-A^{-1} N\left(t_{0}\right)^{-1} .
$$

Suppose to the contrary that (4.11) failed. Then for every $A>0$ there exists $t_{0}=t_{0}(A) \in \mathbb{R}$ such that

$$
\left|x(t)-x\left(t_{0}\right)\right|>\left|t-t_{0}\right|-A^{-1} N\left(t_{0}\right)^{-1}
$$

on $\left[t_{0}, t_{0}+A N\left(t_{0}\right)^{-1}\right]$.

Let $c$ be as in Lemma 4.4, fix $A>c^{-1}$, and choose $t_{0}$ as in (4.12). As a first step, we show

$$
c^{2} N\left(t_{0}\right) \leq N(t) \leq c^{-2} N\left(t_{0}\right) \quad \text { for } \quad t \in\left[t_{0}, t_{0}+A N\left(t_{0}\right)^{-1}\right] .
$$

To obtain (4.13), suppose first that $t \in\left[t_{0}, t_{0}+A N\left(t_{0}\right)^{-1}\right]$ satisfies $N(t) \leq N\left(t_{0}\right)$. By (4.12), we then have the inequality $\left|x(t)-x\left(t_{0}\right)\right|>\left|t-t_{0}\right|-c N\left(t_{0}\right)^{-1} \geq$ $\left|t-t_{0}\right|-c N(t)^{-1}$, so that Lemma 4.4 implies $c^{2} N\left(t_{0}\right) \leq N(t)$. Moreover, the bound $N(t) \leq c^{-2} N\left(t_{0}\right)$ follows trivially from $c<1$, verifying (4.13) in this case. On the other hand, when $N(t)>N\left(t_{0}\right)$, the bound $c^{2} N\left(t_{0}\right) \leq N(t)$ is immediate, and observing that (4.12) implies $\left|x(t)-x\left(t_{0}\right)\right|>\left|t-t_{0}\right|-c N\left(t_{0}\right)^{-1}$, we invoke Lemma 4.4 again to obtain $N(t) \leq c^{-2} N\left(t_{0}\right)$ as desired. Thus, (4.13) holds.

Having obtained (4.13), we now continue towards obtaining the desired contradiction to prove the theorem. Note that by space and time translation symmetries it suffices to assume $t_{0}=0$ and $x\left(t_{0}\right)=0$. We will obtain the desired contradiction by obtaining incompatible lower and upper bounds on

$$
\int_{\beta N(0)^{-1}}^{A N(0)^{-1}} \int_{|| x|-t| \leq \beta N(0)^{-1}}\left|u_{\geq 8 N}(t, x)\right|^{4} d x d t
$$

for a suitable value of $\beta>0$ to be chosen later in the argument.

We first obtain the lower bound on (4.14). Toward this end, we first obtain the set inclusion

$$
\{x:|x-x(t)| \leq C / N(t)\} \subset\left\{x:|| x|-t| \leq \beta N(0)^{-1}\right\} .
$$


To show (4.15), we note that for $t_{0}=0$ and $x\left(t_{0}\right)=0$, (4.12) becomes

$$
|x(t)|>|t|-A^{-1} N(0)^{-1}>|t|-c N(0)^{-1} \text { for } \quad 0 \leq t \leq A N(0)^{-1} .
$$

On the other hand, (2.3) and (4.13) imply

$$
|x(t)| \leq|t|+C\left(N(t)^{-1}+N(0)^{-1}\right) \leq|t|+C N(0)^{-1},
$$

and we therefore conclude that for all $t \in\left[0, A N(0)^{-1}\right]$,

$$
|| x(t)|-t| \lesssim_{u} N(0)^{-1} \text {. }
$$

We now use (4.16) to observe that the inequality $|x-x(t)| \leq C / N(t)$ gives

$$
\begin{aligned}
|| x|-t| & =|| x|-| x(t)||+|| x(t)|-t| \\
& \leq|x-x(t)|+|| x(t)|-t| \\
& \lesssim{ }_{u} N(t)^{-1}+N(0)^{-1} \\
& \lesssim{ }_{u}\left(c^{-2}+1\right) N(0)^{-1},
\end{aligned}
$$

where the implied constant is independent of $A$. We may therefore conclude (4.15) when $\beta$ is chosen sufficiently large (independent of $A$ ). Then, invoking Lemma 4.3 , we obtain

$$
\begin{aligned}
\text { (4.14) } & \geq \int_{\beta N(0)^{-1}}^{A N(0)^{-1}} \int_{|x-x(t)| \leq C / N(t)}\left|u_{\geq 8 N}(t, x)\right|^{4} d x d t \\
& { }_{u} \int_{\beta N(0)^{-1}}^{A N(0)^{-1}} N(t)^{-1} d t \\
& { }_{u} c^{2}(A-\beta) N(0)^{-2},
\end{aligned}
$$

which establishes the desired lower bound on (4.14),

We now establish the upper bound on (4.14). Using Lemma 4.2 followed by the change of variables $z=x-y$ in the $x$ variable, Fubini's theorem, and the change of variables $y^{\prime}=z+y$ in the $y$ variable, we obtain

$$
\begin{aligned}
& {\left[2 \beta N(0)^{-1}\right]^{5}\left(N^{-1}+\eta(A-\beta) N(0)^{-1}\right)} \\
& \quad{ }_{u} \int_{|y| \leq 2 \beta N(0)^{-1}} \int_{\beta N(0)^{-1}}^{A N(0)^{-1}} \int_{|x-y|=t}\left|u_{\geq N}(t, x)\right|^{4} d S(x) d t d y \\
& \quad{ }_{u} \int_{\beta N(0)^{-1}}^{A N(0)^{-1}} \int_{|z|=t} \int_{\left|y^{\prime}-z\right| \leq 2 \beta N(0)^{-1}}\left|u_{\geq N}(t, x)\right|^{4} d y^{\prime} d S(z) d t
\end{aligned}
$$

for $N$ sufficiently small. Observing the inequality

$$
\int_{|z|=t} \chi_{\left\{z:\left|z-y^{\prime}\right| \leq 2 \beta N(0)^{-1}\right\}}(z) d S(z) \gtrsim\left[\beta N(0)^{-1}\right]^{4}
$$

for $t \geq \beta N(0)^{-1}$ and $y^{\prime} \in\left\{|| y^{\prime}|-t| \leq \beta N(0)^{-1}\right\}$, we therefore get

$$
\text { (4.14) } \lesssim{ }_{u} 2^{5} \beta N(0)^{-1} N^{-1}+\eta 2^{5} \beta(A-\beta) N(0)^{-2} \text {. }
$$

Combining the upper and lower bounds (4.18) and (4.17) for (4.14) and choosing $\eta$ sufficiently small (depending on the implicit constant and $\beta$ ), we then have

$$
(A-\beta) N(0)^{-2} \lesssim_{u} 2^{5} \beta N(0)^{-1} N^{-1} .
$$


Note that the implicit constant in (4.19) depends only on the a priori bound $\left\|\left(u, u_{t}\right)\right\|_{L_{t}^{\infty}\left(\dot{H}_{x}^{3 / 2} \times \dot{H}_{x}^{1 / 2}\right)}$ and the compactness modulus function, which are invariant under scaling. In particular, using the scaling of the equation we may assume that $N(0)=1$. Letting $A \rightarrow \infty$ then gives the desired contradiction.

\section{IMPROVED DECAY PROPERTIES}

The main goal of this section is to complete the proof of the following theorem, which states that the soliton-like and low-to-high frequency cascade solutions have finite energy.

Theorem 5.1. Assume that $u: \mathbb{R} \times \mathbb{R}^{5} \rightarrow \mathbb{R}$ is an almost periodic solution to (NLW) with $\left(u, u_{t}\right) \in L_{t}^{\infty}\left(\mathbb{R} ; \dot{H}_{x}^{3 / 2} \times \dot{H}_{x}^{1 / 2}\right)$ and

$$
\inf _{t \in I} N(t) \geq 1 \text {. }
$$

Then $\left(u, u_{t}\right) \in L_{t}^{\infty}\left(\mathbb{R} ; \dot{H}_{x}^{1} \times L_{x}^{2}\right)$ and, moreover, there exist $\beta>0$ and $N_{1}>0$ such that

$$
\left\|\langle x-x(t)\rangle^{\beta} P_{\leq N_{1}} \nabla u\right\|_{L_{t}^{\infty} L_{x}^{2}}+\left\|\langle x-x(t)\rangle^{\beta} P_{\leq N_{1}} u_{t}\right\|_{L_{t}^{\infty} L_{x}^{2}}<\infty .
$$

Theorem 5.1 will be proved by establishing a slight improvement of the decay properties of $u$, in which bounds of the form $\left(u, u_{t}\right) \in L_{t}^{\infty}\left(\dot{H}_{x}^{s} \times \dot{H}_{x}^{s-1}\right)$ are shown to imply $\left(u, u_{t}\right) \in L_{t}^{\infty}\left(\dot{H}_{x}^{s-\epsilon} \times \dot{H}_{x}^{s-1-\epsilon}\right)$ for $\epsilon>0$ sufficiently small (see Lemma 5.1 below). Theorem 5.1 is then proven by an iterative application of Lemma 5.1. starting with the a priori bound $\left(u, u_{t}\right) \in L_{t}^{\infty}\left(\dot{H}_{x}^{s_{c}} \times \dot{H}_{x}^{s_{c}-1}\right)$.

More precisely, we obtain

Lemma 5.1. Assume that $u: \mathbb{R} \times \mathbb{R}^{5} \rightarrow \mathbb{R}$ is a global almost periodic solution to (NLW) with $\left(u, u_{t}\right) \in L_{t}^{\infty}\left(\mathbb{R} ; \dot{H}_{x}^{3 / 2} \times \dot{H}_{x}^{1 / 2}\right)$ and

$$
\inf _{t \in I} N(t) \geq 1 \text {. }
$$

Then there exist constants $\epsilon_{0}, \beta>0$ and a dyadic number $N_{1}>0$ such that for any $s \in\left(1, \frac{3}{2}\right]$, the condition

$$
\left(u, u_{t}\right) \in L_{t}^{\infty}\left(\mathbb{R} ; \dot{H}_{x}^{s} \times \dot{H}_{x}^{s-1}\right)
$$

implies

$$
\left\|\langle x-x(t)\rangle^{\beta} P_{\leq N_{1}}|\nabla|^{s-\epsilon} u\right\|_{L_{t}^{\infty} L_{x}^{2}}+\left\|\langle x-x(t)\rangle^{\beta} P_{\leq N_{1}}|\nabla|^{s-1-\epsilon} u_{t}\right\|_{L_{t}^{\infty} L_{x}^{2}}<\infty
$$

for every $0<\epsilon<\epsilon_{0}$ satisfying $s-\epsilon \geq 1$.

In particular, this allows us to conclude

$$
\left(u, u_{t}\right) \in L_{t}^{\infty}\left(\mathbb{R} ; \dot{H}_{x}^{s-\epsilon} \times \dot{H}_{x}^{s-1-\epsilon}\right)
$$

for any such $\epsilon$.

Proof. As in [21, we begin by observing that to prove the claim it suffices to obtain an estimate of the form

$$
\left\|P_{\leq N_{1}}|\nabla|^{s-\epsilon} u(0)\right\|_{L_{x}^{2}\left(B_{R}\right)}+\left\|P_{\leq N_{1}}|\nabla|^{s-1-\epsilon} u_{t}(0)\right\|_{L_{x}^{2}\left(B_{R}\right)} \lesssim u R^{-\beta}
$$

for some $\beta>0$ and all balls $B_{R}=\left\{x:\left|x-x_{0}\right|<R\right\}$ with $x_{0} \in \mathbb{R}^{5}$ and $\left|x_{0}\right|=3 R$.

Indeed, using the time and space translation symmetries to reduce the desired estimate (5.1) to the case $t=0, x(0)=0$, a covering argument by Whitney balls (that is, balls of the form $B_{R}$ ) shows that (5.1) follows from (5.3). 
To see that (5.2) follows from (5.1), fix $\epsilon \in(0, s-1]$. Decomposing the solution into low and high frequencies, and using Bernstein's inequality, we obtain

$$
\begin{aligned}
& \left\||\nabla|^{s-\epsilon} u\right\|_{L_{t}^{\infty} L_{x}^{2}}+\left\||\nabla|^{s-1-\epsilon} u_{t}\right\|_{L_{t}^{\infty} L_{x}^{2}} \\
& \leq\left\||\nabla|^{s-\epsilon} P_{\leq N_{1}} u\right\|_{L_{t}^{\infty} L_{x}^{2}}+\left\||\nabla|^{s-1-\epsilon} \partial_{t} P_{\leq N_{1}} u\right\|_{L_{t}^{\infty} L_{x}^{2}} \\
& \quad+\sum_{N>N_{1}}\left\||\nabla|^{s-\epsilon} u_{N}\right\|_{L_{t}^{\infty} L_{x}^{2}}+\left\||\nabla|^{s-1-\epsilon} \partial_{t} u_{N}\right\|_{L_{t}^{\infty} L_{x}^{2}} \\
& \quad \lesssim\left\||\nabla|^{s-\epsilon} P_{\leq N_{1}} u\right\|_{L_{t}^{\infty} L_{x}^{2}}+\left\||\nabla|^{s-1-\epsilon} \partial_{t} P_{\leq N_{1}} u\right\|_{L_{t}^{\infty} L_{x}^{2}}+\sum_{N>N_{1}} N^{s-\epsilon-\frac{3}{2}} \\
& \quad \lesssim\left\||\nabla|^{s-\epsilon} P_{\leq N_{1}} u\right\|_{L_{t}^{\infty} L_{x}^{2}}+\left\||\nabla|^{s-1-\epsilon} \partial_{t} P_{\leq N_{1}} u\right\|_{L_{t}^{\infty} L_{x}^{2}}+1,
\end{aligned}
$$

where to obtain the second inequality we have used the a priori bound $\left(u, u_{t}\right) \in$ $L_{t}^{\infty}\left(\dot{H}_{x}^{3 / 2} \times \dot{H}_{x}^{1 / 2}\right)$.

Thus, in order to obtain (5.1) and (5.2), it suffices to show (5.3).

Recalling the definition of $\theta$ in (2.6), we now choose $0<\eta<2$ such that $\theta(\xi) \geq \frac{1}{2}$ for all $|\xi|<\eta$, and a dyadic number $N_{1}$ such that $N_{1} \leq \frac{\eta}{2}$. Let $\phi_{R}$ be given by

$$
\phi_{R}(x)=\phi\left(\frac{x-x_{0}}{R}\right),
$$

and note that $N_{1} \leq \frac{\eta}{2}$ gives supp $\widehat{P_{\leq N_{1}} f} \subset\{\xi:|\xi| \leq \eta\}$ for $f \in L^{2}$, and thus

$$
\left\|P_{\leq N_{1}} f\right\|_{L_{x}^{2}} \lesssim\left\|\theta(i \nabla) P_{\leq N_{1}} f\right\|_{L_{x}^{2}} .
$$

Using the Duhamel formula of Proposition 1.2 forward and backward in time, we obtain

$$
\begin{aligned}
& \left\|P_{\leq N_{1}}|\nabla|^{s-\epsilon} u(0)\right\|_{L_{x}^{2}\left(B_{R}\right)}+\left\|P_{\leq N_{1}}|\nabla|^{s-1-\epsilon} u_{t}(0)\right\|_{L_{x}^{2}\left(B_{R}\right)} \\
& \lesssim\left\|\theta(i \nabla) P_{\leq N_{1}}|\nabla|^{s-\epsilon} u(0)\right\|_{L_{x}^{2}\left(B_{R}\right)}^{2}+\left\|\theta(i \nabla) P_{\leq N_{1}}|\nabla|^{s-1-\epsilon} \partial_{t} u(0)\right\|_{L_{x}^{2}\left(B_{R}\right)}^{2} \\
& \lesssim-\left\langle\int_{0}^{\infty} \nabla \frac{\sin (-t|\nabla|)}{|\nabla|} \theta(i \nabla)|\nabla|^{s-1-\epsilon} P_{\leq N_{1}} F(u(t)) d t,\right. \\
& \left.\phi_{R} \int_{-\infty}^{0} \nabla \frac{\sin (-\tau|\nabla|)}{|\nabla|} \theta(i \nabla)|\nabla|^{s-1-\epsilon} P_{\leq N_{1}} F(u(\tau)) d \tau\right\rangle \\
& -\left\langle\int_{0}^{\infty} \cos (-t|\nabla|) \theta(i \nabla)|\nabla|^{s-1-\epsilon} P_{\leq N_{1}} F(u(t)) d t,\right. \\
& \left.\phi_{R} \int_{-\infty}^{0} \cos (-\tau|\nabla|) \theta(i \nabla)|\nabla|^{s-1-\epsilon} P_{\leq N_{1}} F(u(\tau)) d \tau\right\rangle .
\end{aligned}
$$

In order to obtain bounds for the space-time integrals in (5.5), following [21], we now define the cutoff functions $\rho_{R}, \sigma_{R}, \tilde{\rho_{R}}$ and $\tilde{\sigma_{R}}$.

Lemma 5.2 (Specification of the cutoffs). There exists $R_{0}>1$ such that for every $R>R_{0}$ there exist cutoff functions $\rho_{R}, \sigma_{R}$ and $\tilde{\rho_{R}}, \tilde{\sigma_{R}} \in C^{\infty}\left(\mathbb{R} \times \mathbb{R}^{5} ;[0,1]\right)$ such that

(i) for all $(t, x) \in \mathbb{R} \times \operatorname{supp} \phi_{R}$,

$$
\begin{aligned}
\frac{\sin (-t|\nabla|)}{|\nabla|} & \theta(i \nabla)|\nabla|^{s-1-\epsilon} P_{\leq N_{1}} F(u(t)) \\
= & \frac{\sin (-t|\nabla|)}{|\nabla|} \theta(i \nabla) \rho_{R}(t, x)|\nabla|^{s-1-\epsilon} P_{\leq N_{1}} F(u(t))
\end{aligned}
$$


and

$$
\begin{aligned}
& \cos (-t|\nabla|) \theta(i \nabla)|\nabla|^{s-1-\epsilon} P_{\leq N_{1}} F(u(t)) \\
& \quad=\cos (-t|\nabla|) \theta(i \nabla) \rho_{R}(t, x)|\nabla|^{s-1-\epsilon} P_{\leq N_{1}} F(u(t)),
\end{aligned}
$$

(ii) for all $(t, x) \in\left\{(t, x): t<\frac{R}{2}\right.$ or $\left.t>\frac{10}{\delta} R\right\}$,

$$
\sigma_{R}(t, x)=1 \text {, }
$$

(iii) there exists $C_{1}>0$ such that for all $t \in \mathbb{R}$ and $\delta$ as in Theorem 4.1,

$$
\operatorname{dist}\left(\operatorname{supp} \rho_{R} \sigma_{R}(t), \operatorname{supp}\left(1-\tilde{\rho_{R}} \tilde{\sigma_{R}}(t)\right)\right) \geq C_{1}(|t|+R)
$$

and

$$
\operatorname{dist}\left(x(t), \operatorname{supp}\left(\tilde{\sigma_{R}} \tilde{\rho_{R}}(t)\right)\right) \geq C_{1}(|t|+R),
$$

(iv) there exists $C_{2}>0$ such that

$$
\left\|\nabla \sqrt{\rho_{R} \sigma_{R}}\right\|_{L_{t}^{\infty} L_{x}^{5}} \leq C_{2}, \quad \text { and } \quad\left\|\nabla\left[\rho_{R}\left(1-\sigma_{R}\right)\right]\right\|_{L_{t}^{\infty} L_{x}^{5}} \leq C_{2},
$$

(v) there exists $C_{3}>0$ such that for all $\tau<0<t$ and $x, y \in \mathbb{R}^{5}$ with $(t, x),(\tau, y) \in \operatorname{supp}\left(1-\sigma_{R}\right)$,

$$
|t|+|\tau|+|x|+|y| \leq C_{3} R \quad \text { and } \quad|t-\tau|-|x-y| \geq C_{3} R .
$$

Proof. Fix $R_{0} \geq 1$ to be determined later in the argument, and let $R>R_{0}$ be given. Recall that $x_{0} \in \mathbb{R}^{5}$ is an arbitrary point satisfying $\left|x_{0}\right|=3 R$.

We begin by choosing $\rho_{R}$ such that $\rho_{R}(t, x)=1$ on $\left\{(t, x) \in \mathbb{R} \times \mathbb{R}^{5}:|| x-x_{0} \mid-\right.$ $\left.|t| \mid \leq \frac{6}{5} R\right\}$ and

$$
\operatorname{supp} \rho_{R} \subset\left\{(t, x):\left(1-\frac{\delta}{10^{6}}\right)|t|-\frac{6}{5} R \leq\left|x-x_{0}\right| \leq\left(1+\frac{\delta}{10^{6}}\right)|t|+\frac{6}{5} R\right\},
$$

along with the condition that for each multi-index $\alpha=\left(\alpha_{1}, \cdots, \alpha_{5}\right)$, there exists $C_{\alpha}>0$ such that

$$
\left|\partial_{x}^{\alpha} \rho_{R}\right| \leq C_{\alpha}(|t|+R)^{-|\alpha|} .
$$

Similarly, we choose $\sigma_{R}$ such that $\sigma_{R}=1$ on $\left\{(t, x): t \in\left(-\infty, \frac{R}{2}\right) \cup\left(\frac{10}{\delta} R, \infty\right)\right.$ or $\left.|x-x(t)|>\frac{\delta}{5}(|t|+R)\right\}$ and

$$
\operatorname{supp} \sigma_{R} \subset\left\{(t, x): t \in\left(-\infty, \frac{R}{2}\right) \cup\left(\frac{10}{\delta} R, \infty\right) \text { or }|x-x(t)|>\frac{\delta}{10}(|t|+R)\right\}
$$

along with the derivative bound

$$
\left|\partial_{x}^{\alpha} \sigma_{R}\right|+\left|\partial_{x}^{\alpha}\left(1-\sigma_{R}\right)\right| \leq C_{\alpha}(|t|+R)^{-|\alpha|}
$$

for each multi-index $\alpha$.

Next, we choose $\tilde{\rho}_{R}$ such that $\tilde{\rho}_{R}=1$ on $\left\{(t, x): \operatorname{dist}\left(x,\left\{x: x \in \operatorname{supp}_{x} \rho_{R}(t, x)\right\}\right)\right.$ $\left.\leq \frac{1}{10} R+\frac{\delta}{10^{6}}|t|\right\}$, where $\operatorname{supp}_{x}$ denotes the support in $x$, and

$$
\operatorname{supp} \tilde{\rho}_{R} \subset\left\{(t, x):\left(1-\frac{3 \delta}{10^{6}}\right)|t|-\frac{8}{5} R \leq\left|x-x_{0}\right| \leq\left(1+\frac{3 \delta}{10^{6}}\right)|t|+\frac{8}{5} R\right\}
$$

along with the derivative bound

$$
\left|\partial_{x}^{\alpha} \tilde{\rho}_{R}\right| \leq C_{\alpha}(|t|+R)^{-|\alpha|}
$$

for each multi-index $\alpha$. 
To finish the construction, we now specify $\tilde{\sigma}_{R}$. In particular, we choose $\tilde{\sigma}_{R}$ such that $\tilde{\sigma}_{R}=1$ on $\left\{(t, x): \operatorname{dist}\left(x, \operatorname{supp}_{x} \sigma_{R}(t)\right) \leq \frac{\delta}{40}(|t|+R)\right\}$ and

$$
\operatorname{supp}_{x} \tilde{\sigma}_{R}(t) \subset\left\{x:|x-x(t)| \geq \frac{\delta}{20}(|t|+R)\right\}
$$

for each $t \in\left[\frac{R}{2}, \frac{10}{\delta} R\right]$, as well as the derivative bounds

$$
\left|\partial_{x}^{\alpha} \tilde{\sigma}_{R}\right| \leq C_{\alpha}(|t|+R)^{-|\alpha|}
$$

for each multi-index $\alpha$.

The properties (i)-(v) are now easily verified: noting that supp $\widehat{\theta} \subset\left\{x:\left|x_{i}\right| \leq\right.$ $4, i=1, \cdots, 5\}$, (i) follows from the representations (2.1) and (2.2) for the linear propagator (in particular, this is a formulation of Huygens' principle). Properties (ii) and (iii) then follow directly from the construction, while (iv) follows from the derivative bounds given in the construction of each function, after a suitable change of variables.

To conclude (v), we note $\operatorname{supp}\left(1-\sigma_{R}\right) \subset\left\{(t, x):|t| \in\left[\frac{R}{2}, \frac{10}{\delta} R\right]\right\}$, and thus there exists $C>0$ such that $|t|+|\tau| \leq C R$ for $(t, x),(\tau, y) \in \operatorname{supp}\left(1-\sigma_{R}\right)$. The finite speed of propagation in the form of Lemma 2.1 then implies

$$
|x(t)| \leq|x(t)-x(0)|+|x(0)| \leq|t|+|x(0)|+C
$$

for all $t \in \mathbb{R}$, and thus for all $(t, x) \in \operatorname{supp}\left(1-\sigma_{R}\right)$ we have

$$
|x| \leq|x-x(t)|+|x(t)| \leq \frac{\delta}{5}(|t|+R)+|t|+C \leq C R
$$

for some $C>0$, where we have used the facts $R \geq 1$ and $\sigma_{R}=1$ on $\{(t, x)$ : $\left.|x-x(t)|>\frac{\delta}{5}(|t|+R)\right\}$. We therefore conclude that there exists $C>0$ such that for all $(t, x),(\tau, y) \in \operatorname{supp}\left(1-\sigma_{R}\right)$,

$$
|t|+|\tau|+|x|+|y| \leq C R
$$

which is the first component of (v).

To see that the second component holds, we fix $R_{0}>\max \left\{1, \delta^{-1}\right\}$ and apply Theorem 4.1 to obtain

$$
\begin{aligned}
|t-\tau|-|x-y| & \geq \delta|t-\tau|-|x-x(t)|-|x(\tau)-y| \\
& \geq \frac{2 \delta}{5} R,
\end{aligned}
$$

where we observe that $(t, x),(\tau, y) \in \operatorname{supp}\left(1-\sigma_{R}\right)$ with $\tau<0<t$ gives the bounds $|t-\tau| \geq R,|x-x(t)| \leq \frac{\delta}{5}(t+R)$ and $|y-x(\tau)| \leq \frac{\delta}{5}(-\tau+R)$.

This completes the proof of Lemma 5.2 . 
Having specified the cutoffs, we now use these functions to decompose the spatial integration given by the inner product in (5.5), obtaining

$$
\begin{gathered}
(15.5) \lesssim-\left\langle\int_{0}^{\infty} \nabla \frac{\sin (-t|\nabla|)}{|\nabla|} \theta(i \nabla) \rho_{R}(t, x)|\nabla|^{s-1-\epsilon} P_{\leq N_{1}} F(u(t)),\right. \\
\left.\phi_{R} \int_{-\infty}^{0} \nabla \frac{\sin (-\tau|\nabla|)}{|\nabla|} \theta(i \nabla) \rho_{R}(t, x)|\nabla|^{s-1-\epsilon} P_{\leq N_{1}} F(u(\tau))\right\rangle \\
-\left\langle\int_{0}^{\infty} \cos (-t|\nabla|) \theta(i \nabla) \rho_{R}(t, x)|\nabla|^{s-1-\epsilon} P_{\leq N_{1}} F(u(t)),\right. \\
\left.\phi_{R} \int_{-\infty}^{0} \cos (-\tau|\nabla|) \theta(i \nabla) \rho_{R}(t, x)|\nabla|^{s-1-\epsilon} P_{\leq N_{1}} F(u(\tau))\right\rangle \\
=-\left\langle A_{1}^{+}, \phi_{R} A_{1}^{-}\right\rangle-\left\langle A_{1}^{+}, \phi_{R} A_{2}^{-}\right\rangle-\left\langle A_{2}^{+}, \phi_{R} A_{1}^{-}\right\rangle-\left\langle A_{2}^{+}, \phi_{R} A_{2}^{-}\right\rangle \\
-\left\langle B_{1}^{+}, \phi_{R} B_{1}^{-}\right\rangle-\left\langle B_{1}^{+}, \phi_{R} B_{2}^{-}\right\rangle-\left\langle B_{2}^{+}, \phi_{R} B_{1}^{-}\right\rangle-\left\langle B_{2}^{+}, \phi_{R} B_{2}^{-}\right\rangle \\
\leq\left\|A_{1}^{+}\right\|_{L_{x}^{2}}\left\|A_{1}^{-}\right\|_{L_{x}^{2}}+\left\|A_{1}^{+}\right\|_{L_{x}^{2}}\left\|A_{2}^{-}\right\|_{L_{x}^{2}}+\left\|A_{2}^{+}\right\|_{L_{x}^{2}}\left\|A_{1}^{-}\right\|_{L_{x}^{2}} \\
+\left\|B_{1}^{+}\right\|_{L_{x}^{2}}\left\|B_{1}^{-}\right\|_{L_{x}^{2}}+\left\|B_{1}^{+}\right\|_{L_{x}^{2}}\left\|B_{2}^{-}\right\|_{L_{x}^{2}}+\left\|B_{2}^{+}\right\|_{L_{x}^{2}}\left\|B_{1}^{-}\right\|_{L_{x}^{2}} \\
+\left|\left\langle A_{2}^{+}, \phi_{R} A_{2}^{-}\right\rangle\right|+\left|\left\langle B_{2}^{+}, \phi_{R} B_{2}^{-}\right\rangle\right|,
\end{gathered}
$$

where we have set

$$
\begin{aligned}
& A_{1}^{+}(x):=\int_{0}^{\infty} \nabla \frac{\sin (-t|\nabla|)}{|\nabla|} \theta(i \nabla) \rho_{R}(t, x) \sigma_{R}(t, x)|\nabla|^{s-1-\epsilon} P_{\leq N_{1}} F(u(t)) d t, \\
& A_{1}^{-}(x):=\int_{-\infty}^{0} \nabla \frac{\sin (-\tau|\nabla|)}{|\nabla|} \theta(i \nabla) \rho_{R}(\tau, x) \sigma_{R}(\tau, x)|\nabla|^{s-1-\epsilon} P_{\leq N_{1}} F(u(\tau)) d \tau, \\
& A_{2}^{+}(x):=\int_{0}^{\infty} \nabla \frac{\sin (-t|\nabla|)}{|\nabla|} \theta(i \nabla) \rho_{R}(t, x)\left(1-\sigma_{R}(t, x)\right)|\nabla|^{s-1-\epsilon} P_{\leq N_{1}} F(u(t)) d t, \\
& A_{2}^{-}(x):=\int_{-\infty}^{0} \nabla \frac{\sin (-\tau|\nabla|)}{|\nabla|} \theta(i \nabla) \rho_{R}(\tau, x)\left(1-\sigma_{R}(\tau, x)\right)|\nabla|^{s-1-\epsilon} P_{\leq N_{1}} F(u(\tau)) d \tau,
\end{aligned}
$$

as well as

$$
\begin{aligned}
& B_{1}^{+}(x):=\int_{0}^{\infty} \cos (-t|\nabla|) \theta(i \nabla) \rho_{R}(t, x) \sigma_{R}(t, x)|\nabla|^{s-1-\epsilon} P_{\leq N_{1}} F(u(t)) d t, \\
& B_{1}^{-}(x):=\int_{-\infty}^{0} \cos (-\tau|\nabla|) \theta(i \nabla) \rho_{R}(\tau, x) \sigma_{R}(\tau, x)|\nabla|^{s-1-\epsilon} P_{\leq N_{1}} F(u(\tau)) d \tau, \\
& B_{2}^{+}(x):=\int_{0}^{\infty} \cos (-t|\nabla|) \theta(i \nabla) \rho_{R}(t, x)\left(1-\sigma_{R}(t, x)\right)|\nabla|^{s-1-\epsilon} P_{\leq N_{1}} F(u(t)) d t, \\
& B_{2}^{-}(x):=\int_{-\infty}^{0} \cos (-\tau|\nabla|) \theta(i \nabla) \rho_{R}(\tau, x)\left(1-\sigma_{R}(\tau, x)\right)|\nabla|^{s-1-\epsilon} P_{\leq N_{1}} F(u(\tau)) d \tau .
\end{aligned}
$$

The remainder of the proof of Lemma 5.1 now consists of estimating the norms and inner products appearing in (5.8). We accomplish this in the following three propositions.

Proposition 5.1. We have

$$
\max \left\{\left\|A_{1}^{+}\right\|_{L_{x}^{2}},\left\|A_{1}^{-}\right\|_{L_{x}^{2}},\left\|B_{1}^{+}\right\|_{L_{x}^{2}},\left\|B_{1}^{-}\right\|_{L_{x}^{2}}\right\} \lesssim R^{-1 / 2-\beta}
$$

for every $\beta<\frac{1}{1000}$, provided that $\epsilon>0$ is sufficiently small. 
Proof. We argue as in [21]. We show the estimate for $\left\|A_{1}^{+}\right\|_{L_{x}^{2}}$ and remark that the other estimates are similar. Using the inhomogeneous Strichartz inequality and the fractional product rule followed by Hölder's inequality, we obtain

$\left\|A_{1}^{+}\right\|_{L_{x}^{2}}$

$$
\begin{aligned}
& \leq \lim _{T \rightarrow \infty}\left\|\int_{0}^{T}|\nabla|^{5 / 4} \frac{\sin (-t|\nabla|)}{|\nabla|} \theta(i \nabla) \rho_{R}(t, x) \sigma_{R}(t, x)|\nabla|^{s-\frac{5}{4}-\epsilon} P_{\leq N_{1}} F(u(t)) d t\right\|_{L_{x}^{2}} \\
& \lesssim\left\|\nabla\left[\rho_{R}(t, x) \sigma_{R}(t, x)|\nabla|^{s-\frac{5}{4}-\epsilon} P_{\leq N_{1}} F(u(t))\right]\right\|_{L_{t}^{2} L_{x}^{4 / 3}\left(\mathbb{R} \times \mathbb{R}^{5}\right)} \\
& \lesssim\left\|\left[\nabla\left[\rho_{R} \sigma_{R}\right]\right]|\nabla|^{s-\frac{5}{4}-\epsilon} P_{\leq N_{1}} F(u(t))\right\|_{L_{t}^{2} L_{x}^{4 / 3}} \\
& \quad+\left\|\rho_{R} \sigma_{R} \nabla|\nabla|^{s-\frac{5}{4}-\epsilon} P_{\leq N_{1}} F(u(t))\right\|_{L_{t}^{2} L_{x}^{4 / 3}} \\
& \lesssim\left\|\nabla\left[\rho_{R}^{1 / 2} \sigma_{R}^{1 / 2}\right]\right\|_{L_{t}^{\infty} L_{x}^{5}}\left\|\rho_{R}^{1 / 2} \sigma_{R}^{1 / 2}|\nabla|^{s-\frac{5}{4}-\epsilon} P_{\leq N_{1}} F(u(t))\right\|_{L_{t}^{2} L_{x}^{20 / 11}}
\end{aligned}
$$

$$
+\left\|\rho_{R}^{1 / 2} \sigma_{R}^{1 / 2}|\nabla|^{s-\frac{1}{4}-\epsilon} P_{\leq N_{1}} F(u(t))\right\|_{L_{t}^{2} L_{x}^{4 / 3}} .
$$

Observing the identity $F(x)=F\left(\tilde{\rho_{R}} \tilde{\sigma_{R}} x\right)+\left(1-{\tilde{\rho_{R}}}^{3}{\tilde{\sigma_{R}}}^{3}\right) F(x)$, we obtain

$$
\begin{aligned}
\left\|A_{1}^{+}\right\|_{L_{x}^{2}} \lesssim & \left\|\rho_{R}^{1 / 2} \sigma_{R}^{1 / 2}|\nabla|^{s-\frac{5}{4}-\epsilon} P_{\leq N_{1}} F\left(\tilde{\rho_{R}} \tilde{\sigma_{R}} u(t)\right)\right\|_{L_{t}^{2} L_{x}^{20 / 11}} \\
& +\left\|\rho_{R}^{1 / 2} \sigma_{R}^{1 / 2}|\nabla|^{s-\frac{5}{4}-\epsilon} P_{\leq N_{1}}\left(1-\tilde{\rho_{R}}{ }^{3}{\tilde{\sigma_{R}}}^{3}\right) F(u(t))\right\|_{L_{t}^{2} L_{x}^{20 / 11}} \\
& +\left\|\rho_{R}^{1 / 2} \sigma_{R}^{1 / 2} \nabla|\nabla|^{s-\frac{5}{4}-\epsilon} P_{\leq N_{1}} F\left(\tilde{\rho_{R}} \tilde{\sigma_{R}} u(t)\right)\right\|_{L_{t}^{2} L_{x}^{4 / 3}} \\
& +\left\|\rho_{R}^{1 / 2} \sigma_{R}^{1 / 2} \nabla|\nabla|^{s-\frac{5}{4}-\epsilon} P_{\leq N_{1}}\left(1-\tilde{\rho_{R}} \tilde{\sigma}_{R}^{3}\right) F(u(t))\right\|_{L_{t}^{2} L_{x}^{4 / 3}} .
\end{aligned}
$$

The rest of the proof of Proposition 5.1 is devoted to estimating the terms (5.10)(5.13). To estimate (5.10) and (5.12), we perform a dyadic decomposition in time. The advantage of this decomposition arises out of the following lemma, which allows us to obtain uniform bounds on a localized Strichartz norm of the solution $u$.

Lemma 5.3. There exists $R_{0}>0$ and $C>0$ such that

$$
\left\|\nabla\left(\tilde{\rho_{R}} \tilde{\sigma_{R}} u\right)\right\|_{L_{t}^{3}\left(I ; L_{x}^{3}\right)} \leq C
$$

for every $R>R_{0}$ and $I \subset \mathbb{R}$ of the form $I=\left[-\frac{10}{\delta} R, \frac{10}{\delta} R\right]$ or $I=[T, 2 T]$ with $T \geq \frac{10}{\delta} R$.

We remark that Lemma 5.3 is obtained as in Lemma 7.2 of 21 with an additional derivative which is accounted for by choosing the space-time norm in accordance with the Strichartz inequality. The proof is based on the small data global theory, finite speed of propagation, and the subluminality result, Theorem 4.1, after observing that $\nabla$ is a local operator and therefore behaves well with respect to the finite speed of propagation.

With Lemma 5.3 in hand, we return to the task of estimating (5.10) and (5.12). We set $T_{0}=0, T_{j}=\frac{10}{\delta} R 2^{j-1}, j \geq 1$, and use Lemma $5.2(\mathrm{v})$ along with the Sobolev inequality and the decomposition

$$
\tilde{\rho_{R}} \tilde{\sigma_{R}} u(t)=P_{<8 N_{1}}\left(\tilde{\rho_{R}} \tilde{\sigma_{R}} u(t)\right)+P_{\geq 8 N_{1}}\left(\tilde{\rho_{R}} \tilde{\sigma_{R}} u(t)\right)
$$


to obtain

$$
\begin{aligned}
& \text { (5.10) }+5.12 \\
& \lesssim \sum_{j=0}^{\infty}\left\|P_{<N_{1}}|\nabla|^{s-\epsilon-\frac{1}{4}} F\left(\tilde{\rho_{R}} \tilde{\sigma_{R}} u(t)\right)\right\|_{L_{t}^{2}\left(I_{j} ; L_{x}^{4 / 3}\right)} \\
& \lesssim \sum_{j=0}^{\infty}\left[\left\||\nabla|^{s-\epsilon-\frac{1}{4}}\left[P_{<8 N_{1}}\left(\tilde{\rho_{R}} \tilde{\sigma_{R}} u(t)\right)\right]^{3}\right\|_{L_{t}^{2}\left(I_{j} ; L_{x}^{4 / 3}\right)}\right. \\
& +\left\||\nabla|^{s-\epsilon-\frac{1}{4}}\left[P_{<8 N_{1}}\left(\tilde{\rho_{R}} \tilde{\sigma_{R}} u(t)\right)\right]\left[P_{\geq 8 N_{1}}\left(\tilde{\rho_{R}} \tilde{\sigma_{R}} u(t)\right)\right] \tilde{\rho_{R}} \tilde{\sigma_{R}} u(t)\right\|_{L_{t}^{2}\left(I_{j} ; L_{x}^{4 / 3}\right)} \\
& \left.+\left\|P_{<N_{1}}|\nabla|^{s-\epsilon-\frac{1}{4}}\left[P_{\geq 8 N_{1}}\left(\tilde{\rho_{R}} \tilde{\sigma_{R}} u(t)\right)\right]^{3}\right\|_{L_{t}^{2}\left(I_{j} ; L_{x}^{4 / 3}\right)}\right]
\end{aligned}
$$

$$
=: \sum_{j=0}^{\infty}(I)_{j}+(I I)_{j}+(I I I)_{j}
$$

with $I_{j}=\left[T_{j}, T_{j+1}\right]$. In the interest of simplifying notation for the rest of the proof, all $L_{t}^{q}$ norms in the subsequent argument will be on the interval $I_{j}$.

To bound $(I)_{j}$, we consider two cases: $s>\frac{5}{4}$ and $s \leq \frac{5}{4}$. If $s>\frac{5}{4}$ we use the Hölder inequality in time followed by the fractional product rule, interpolation, (3.1), Proposition 3.1, Proposition 3.2, and the Bernstein inequalities (provided $\left.\epsilon<\frac{7}{150}\right)$ to obtain

$$
\begin{aligned}
&(I)_{j} \lesssim\left(2^{j} R\right)^{\frac{3}{14}}\left\||\nabla|^{s-\epsilon-\frac{1}{4}}\left[P_{\leq 8 N_{1}}\left(\tilde{\rho_{R}} \tilde{\sigma_{R}} u(t)\right)\right]^{3}\right\|_{L_{t}^{7 / 2} L_{x}^{4 / 3}} \\
& \lesssim\left(2^{j} R\right)^{\frac{3}{14}}\left\|P_{\leq 8 N_{1}}\left(\tilde{\rho_{R}} \tilde{\sigma_{R}} u(t)\right)\right\|_{L_{t}^{\infty} L_{x}^{5}}^{199 / 100}\left\|P_{\leq 8 N_{1}}\left(\tilde{\rho_{R}} \tilde{\sigma_{R}} u(t)\right)\right\|_{L_{t}^{\infty} L_{x}^{3}}^{1 / 100} \\
& \cdot\left\||\nabla|^{s-\epsilon-\frac{1}{4}} P_{\leq 8 N_{1}}\left(\tilde{\rho_{R}} \tilde{\sigma_{R}} u(t)\right)\right\|_{L_{t, x}^{3}}^{6 / 7}\left\||\nabla|^{s-\epsilon-\frac{1}{4}} P_{\leq 8 N_{1}}\left(\tilde{\rho_{R}} \tilde{\sigma_{R}} u(t)\right)\right\|_{L_{t}^{\infty} L_{x}^{1 / 7}}^{1500 / 661} \\
& \lesssim {\left[R\left(1+2^{j}\right)\right]^{\frac{3}{14}-\frac{199}{100}\left(\frac{3}{8}-\frac{1}{100}\right)}\left(8 N_{1}\right)^{s-\epsilon-\frac{5}{4}}\left(8 N_{1}\right)^{\frac{1}{150}-\frac{\epsilon}{7}} } \\
& \cdot\left\|\nabla\left(\tilde{\rho_{R}} \tilde{\sigma_{R}} u(t)\right)\right\|_{L_{t, x}^{3}}^{6 / 7}\left\||\nabla|^{s-\epsilon-\frac{1}{4}}\left(\tilde{\rho_{R}} \tilde{\sigma_{R}} u(t)\right)\right\|_{L_{t}^{1 / 7}}^{L_{x}^{\frac{20}{9-4 \epsilon}}} \\
& \lesssim u, N_{1} {\left[R\left(1+2^{j}\right)\right]^{-\frac{51}{100}}\left\||\nabla|^{s}\left(\tilde{\rho_{R}} \tilde{\sigma_{R}} u(t)\right)\right\|_{L_{t}^{\infty} L_{x}^{2}}^{1 / 7} } \\
& \lesssim_{u, N_{1}}\left[R\left(1+2^{j}\right)\right]^{-\frac{51}{100}}\left\||\nabla|^{s} u(t)\right\|_{L_{t}^{\infty} L_{x}^{2}}^{1 / 7}
\end{aligned}
$$

$$
\lesssim_{u, N_{1}}\left[R\left(1+2^{j}\right)\right]^{-\frac{51}{100}}
$$

where to obtain the fourth inequality we use $R \geq 1$, Lemma 5.3 and the Sobolev embedding. Moreover, in obtaining the second-to-last inequality, we invoke the fractional product rule, Sobolev embedding and the bounds (5.6) and (5.7) to obtain the estimate

$$
\begin{aligned}
\left\||\nabla|^{s}\left(\tilde{\rho_{R}} \tilde{\sigma_{R}} u(t)\right)\right\|_{L_{t}^{\infty} L_{x}^{2}} \lesssim & \left\||\nabla|^{s}\left(\tilde{\rho_{R}} \tilde{\sigma_{R}}\right)\right\|_{L_{t}^{\infty} L_{x}^{\frac{5}{s}}}\|u(t)\|_{L_{t}^{\infty} \frac{10}{L_{x}^{5-2 s}}} \\
& +\left\|\left(\tilde{\rho_{R}} \tilde{\sigma_{R}}\right)\right\|_{L_{t, x}^{\infty}}\left\||\nabla|^{s} u(t)\right\|_{L_{t}^{\infty} L_{x}^{2}} \\
& \lesssim\left\||\nabla|^{s} u(t)\right\|_{L_{t}^{\infty} L_{x}^{2}}
\end{aligned}
$$


It now remains to consider the case $s \leq \frac{5}{4}$. Towards this end, fixing $w \in$ $\left(\frac{10(3+2 s)}{15+4 s}, 5\right]$, we proceed in a similar manner as above: using Hölder's inequality in time, followed by the fractional product rule, interpolation, Proposition 3.1. Proposition 3.2. (3.1), and the Bernstein and Sobolev inequalities, we obtain

$$
\begin{aligned}
& (I)_{j} \lesssim\left(2^{j} R\right)^{\frac{3(3-2 s)}{2(11-6 s)}}\left\||\nabla|^{s-\epsilon-\frac{1}{4}}\left[P_{\leq 8 N_{1}}\left(\tilde{\rho_{R}} \tilde{\sigma_{R}} u(t)\right)\right]^{3}\right\|_{L_{t}^{11-6 s} L_{x}^{4 / 3}} \\
& \lesssim\left(2^{j} R\right)^{\frac{3(3-2 s)}{2(11-6 s)}}\left\|P_{\leq 8 N_{1}}\left(\tilde{\rho_{R}} \tilde{\sigma_{R}} u(t)\right)\right\|_{L_{t}^{\infty} L_{x}^{5}}^{199 / 100}\left\|P_{\leq 8 N_{1}}\left(\tilde{\rho_{R}} \tilde{\sigma_{R}} u(t)\right)\right\|_{L_{t}^{\infty} L_{x}^{3}}^{1 / 100} \\
& \cdot\left\||\nabla|^{s-\epsilon-\frac{1}{4}} P_{\leq 8 N_{1}}\left(\tilde{\rho_{R}} \tilde{\sigma_{R}} u(t)\right)\right\|_{L_{t}^{3} L_{x}^{\sigma s}}^{\frac{3}{11-6 s}}\left\||\nabla|^{s-\epsilon-\frac{1}{4}} P_{\leq 8 N_{1}}\left(\tilde{\rho_{R}} \tilde{\sigma_{R}} u(t)\right)\right\|_{L_{t}^{\infty} L_{x}^{\sigma s}}^{\frac{2(4-3 s)}{11-6 s}} \\
& \lesssim u\left(2^{j} R\right)^{\frac{3(3-2 s)}{2(11-6 s)}+\frac{199}{100}\left(-\frac{8(5-w)}{5(12-w)}\right)}\left(8 N_{1}\right)^{\frac{2(4-3 s)}{11-6 s}\left(\frac{9}{4}-\epsilon-\frac{5}{\sigma_{2}}\right)} \\
& \cdot\left\||\nabla|^{s-\epsilon-\frac{1}{4}} P_{\leq 8 N_{1}}\left(\tilde{\rho_{R}} \tilde{\sigma_{R}} u(t)\right)\right\|_{L_{t}^{3} L_{x}^{\sigma} I_{1}^{1}}^{\frac{3}{11-s}}\left\||\nabla|^{s-\epsilon-\frac{1}{4}} P_{\leq 8 N_{1}}\left(\tilde{\rho_{R}} \tilde{\sigma_{R}} u(t)\right)\right\|_{L_{t}^{\infty} L_{x}^{\frac{20}{9-4 \epsilon}}}^{\frac{2(4-3 s)}{11-6 s}} \\
& \lesssim_{u, N_{1}}\left(2^{j} R\right)^{\frac{3(3-2 s)}{2(11-6 s)}+\frac{199}{100}\left(-\frac{8(5-w)}{5(12-w)}\right)} \\
& \cdot\left\|\nabla\left(\tilde{\rho_{R}} \tilde{\sigma_{R}} u(t)\right)\right\|_{L_{t, x}^{3}}^{\frac{3}{11-6 s}}\left\||\nabla|^{s}\left(\tilde{\rho_{R}} \tilde{\sigma_{R}} u(t)\right)\right\|_{L_{t}^{\infty} L_{x}^{2}}^{\frac{2(4-3 s)}{11-6 s}} \\
& \lesssim u, N_{1}\left(2^{j} R\right)^{\frac{3(3-2 s)}{2(11-6 s)}+\frac{199}{100}\left(-\frac{8(5-w)}{5(12-w)}\right)}\left\||\nabla|^{s} u(t)\right\|_{L_{t}^{\infty} L_{x}^{2}}^{\frac{2(4-3 s)}{11-6 s}}
\end{aligned}
$$

$$
\lesssim_{u, N_{1}}\left[R\left(1+2^{j}\right)\right]^{-\frac{501}{1000}}
$$

for $\epsilon<\frac{1}{150}$, where we have set

$$
\sigma_{1}=\frac{60}{5+12 s-12 \epsilon} \quad \text { and } \quad \sigma_{2}=\frac{1500(4-3 s)}{2689-2019 s+450 \epsilon},
$$

chosen $w$ sufficiently close to $\frac{10(3+2 s)}{15+4 s}$, and recalled that we have $R \geq 1$. We remark that in order to apply Bernstein in the third inequality, we have observed $\sigma_{2}>\frac{20}{9-4 \epsilon}$ when $\epsilon<\frac{1}{150}$ and $s \leq \frac{5}{4}$. We also note that to obtain the fifth inequality, we used Lemma 5.3 and (5.16).

It now remains to estimate the terms $(I I)_{j}$ and $(I I I)_{j}$. For this, we use Hölder's inequality in time and space, followed by interpolation and the Bernstein inequalities to obtain

$$
\begin{aligned}
(I I)_{j}+ & (I I I)_{j} \\
\lesssim & N_{1}^{s-\epsilon-\frac{1}{4}}\left(2^{j} R\right)^{\frac{1}{5}}\left\|\tilde{\rho_{R}} \tilde{\sigma_{R}} u(t)\right\|_{L_{t}^{\infty} L_{x}^{5}}^{2}\left\|P_{\geq 8 N_{1}}\left(\tilde{\rho_{R}} \tilde{\sigma_{R}} u(t)\right)\right\|_{L_{t}^{10 / 3} L_{x}^{20 / 7}} \\
\lesssim & N_{1}^{s-\epsilon-\frac{1}{4}}\left(2^{j} R\right)^{\frac{1}{5}}\left\|\tilde{\rho_{R}} \tilde{\sigma_{R}} u(t)\right\|_{L_{t}^{\infty} L_{x}^{5}}^{2} \\
& \cdot\left\|P_{\geq 8 N_{1}}\left(\tilde{\rho_{R}} \tilde{\sigma_{R}} u(t)\right)\right\|_{L_{t, x}^{3}}^{9 / 10}\left\|P_{\geq 8 N_{1}}\left(\tilde{\rho_{R}} \tilde{\sigma_{R}} u(t)\right)\right\|_{L_{t}^{\infty} L_{x}^{2}}^{1 / 10} \\
\lesssim & N_{1}^{\frac{9 s}{10}-\frac{23}{20}-\epsilon}\left[R\left(1+2^{j}\right)\right]^{-\frac{23}{45}}\left\|\nabla\left(\tilde{\rho_{R}} \tilde{\sigma_{R}} u(t)\right)\right\|_{L_{t, x}^{3}}^{9 / 10}\left\||\nabla|^{s}\left(\tilde{\rho_{R}} \tilde{\sigma_{R}} u(t)\right)\right\|_{L_{t}^{\infty} L_{x}^{2}}^{1 / 10} \\
\lesssim & { }_{u, N_{1}}\left[R\left(1+2^{j}\right)\right]^{-\frac{23}{45}},
\end{aligned}
$$

where we have used (3.1) and Proposition 3.2 with $w=3$ to obtain the second-tolast inequality, and Lemma 5.3 and (5.16) to obtain the last inequality. 
Combining (5.15)-(5.17) and (5.18) with (5.14), we obtain

$$
(\underline{5.10})+(\underline{5.12}) \lesssim u, N_{1} R^{-\frac{501}{1000}}
$$

whenever $\epsilon$ is sufficiently small.

We now estimate (5.11) and (5.13). Note that by the Sobolev inequality, we have

$$
\begin{aligned}
\text { (5.11) }+(\underline{15.13)} & \lesssim\left\|\rho_{R}^{1 / 2} \sigma_{R}^{1 / 2}|\nabla|^{s-\epsilon-\frac{1}{4}} P_{\leq N_{1}}\left(1-\tilde{\rho}_{R}^{3} \tilde{\sigma}_{R}^{3}\right) F(u(t))\right\|_{L_{t}^{2} L_{x}^{4 / 3}} \\
& \lesssim\left\|(|t|+R)^{-s+\epsilon-1}\left(1-\tilde{\rho}_{R}^{3} \tilde{\sigma}_{R}^{3}\right) F(u(t))\right\|_{L_{t}^{2} L_{x}^{1}} \\
& \lesssim\left\|(|t|+R)^{-s+\epsilon-1}\right\|_{L_{t}^{2}}\|F(u(t))\|_{L_{t}^{\infty} L_{x}^{1}} \\
& \lesssim u R^{-\frac{1}{2}-(s-\epsilon)} \\
& \lesssim u R^{-\frac{1}{2}-\beta}
\end{aligned}
$$

whenever $\beta<s-\epsilon$, where we have invoked Lemma 2.3 with $\sigma=s-\epsilon-\frac{1}{4}$, $A=C_{1}(|t|+R), q=\frac{4}{3}$ and $p=1$. The claim follows by combining (5.19) and (5.22) and observing that for $\epsilon<\frac{1}{2}, \beta<\frac{1}{1000}$ implies $\beta<\frac{1}{2}<s-\epsilon$. This completes the proof of Proposition 5.1 .

Proposition 5.2. We have the bounds

$$
\max \left\{\left\|A_{2}^{+}\right\|_{L_{x}^{2}},\left\|A_{2}^{-}\right\|_{L_{x}^{2}},\left\|B_{2}^{+}\right\|_{L_{x}^{2}},\left\|B_{2}^{-}\right\|_{L_{x}^{2}}\right\} \lesssim R^{\frac{1}{2}}
$$

Proof. The proof proceeds as in the proof of Lemma 7.4 of [21]. We show the estimate for $\left\|A_{2}^{+}\right\|_{L_{x}^{2}}$ and remark that the other estimates are similar. Setting $I_{R}=$ $\left[\frac{R}{2}, \frac{10 R}{\delta}\right]$, we use the inhomogeneous Strichartz inequality followed by the fractional product rule, Hölder's inequality, and the Sobolev and Bernstein inequalities to obtain

$$
\begin{aligned}
\left\|A_{2}^{+}\right\|_{L_{x}^{2}} & \\
\leq & \lim _{T \rightarrow \infty}\left\|\int_{0}^{T} \nabla \frac{\sin (-t|\nabla|)}{|\nabla|} \rho_{R}(t, x)\left(1-\sigma_{R}(t, x)\right)|\nabla|^{s-1-\epsilon} P_{\leq N_{1}} F(u(t)) d t\right\|_{L_{x}^{2}} \\
& \lesssim\left\|\nabla\left[\rho_{R}(t, x)\left(1-\sigma_{R}(t, x)\right)|\nabla|^{s-1-\epsilon} P_{\leq N_{1}} F(u(t))\right]\right\|_{L_{t}^{2} L_{x}^{5 / 4}\left(I_{R} \times \mathbb{R}^{5}\right)} \\
& \lesssim\left\|\nabla\left[\rho_{R}(t, x)\left(1-\sigma_{R}(t, x)\right)\right]\right\|_{L_{t}^{\infty} L_{x}^{5}\left(I_{R} \times \mathbb{R}^{5}\right)}\left\||\nabla|^{s-1-\epsilon} P_{\leq N_{1}} F(u(t))\right\|_{L_{t}^{2} L_{x}^{5 / 3}\left(I_{R} \times \mathbb{R}^{5}\right)} \\
& \quad+\left\|\rho_{R}\left(1-\sigma_{R}\right)\right\|_{L_{t}^{\infty} L_{x}^{\infty}}\left\||\nabla|^{s-\epsilon} P_{\leq N_{1}} F(u(t))\right\|_{L_{t}^{2} L_{x}^{5 / 4}\left(I_{R} \times \mathbb{R}^{5}\right)} \\
& \lesssim\left\|\left.\nabla\right|^{s-\epsilon} P_{\leq N_{1}} F(u(t))\right\|_{L_{t}^{2} L_{x}^{5 / 4}\left(I_{R} \times \mathbb{R}^{5}\right)} \\
& \lesssim\|F(u(t))\|_{L_{t}^{\infty} L_{x}^{5 / 4}\left|I_{R}\right|^{\frac{1}{2}}} \\
& \lesssim\|u\|_{L_{t}^{\infty} L_{x}^{15 / 4}}^{3} R^{\frac{1}{2}} \\
& \lesssim R^{\frac{1}{2}}
\end{aligned}
$$

where we have used (3.1) interpolated with the a priori bound

$$
\left(u, u_{t}\right) \in L_{t}^{\infty}\left(\dot{H}_{x}^{3 / 2} \times \dot{H}_{x}^{1 / 2}\right)
$$

to obtain the last inequality. 
To estimate the last term in (5.8), we will make use of the following weak diffraction lemma, the proof of which we give in Appendix A. This result is the analogue of the weak diffraction property presented in [21], adapted to the dimension $d=5$ setting.

Lemma 5.4 (Weak diffraction). Fix $\phi \in C^{\infty}\left(\mathbb{R}^{5} ;[0,1]\right)$ such that $\phi(x)=1$ for $|x|<1$ and $\phi(x)=0$ for $|x|>2$. Then there exists $C>0$ such that if $F, G$ : $\mathbb{R} \times \mathbb{R}^{5} \rightarrow \mathbb{R}$ are given along with $R_{0}, C_{1}, C_{2}>0$ such that for every $R \geq R_{0}$ we have

$$
\begin{aligned}
\operatorname{supp} F & \times \operatorname{supp} G \\
& \subset\left\{((t, x),(\tau, y)):|t|+|\tau|+|x|+|y| \leq C_{1} R,|t-\tau|-|x-y| \geq C_{2} R\right\},
\end{aligned}
$$

then for every $R \geq R_{0}$ and $x_{0} \in \mathbb{R}^{5}$ we have

$$
|I(F, G, R)| \leq C R^{-1 / 26}\|F\|_{L_{t}^{\infty} L_{x}^{1}}\|G\|_{L_{t}^{\infty} L_{x}^{1}},
$$

where

$$
\begin{aligned}
I(F, G, R):= & \int_{\mathbb{R}} \int_{\mathbb{R}}\left\langle\nabla \frac{\sin (t|\nabla|)}{|\nabla|} \theta(i \nabla) F(t), \phi\left(\frac{\cdot-x_{0}}{R}\right) \nabla \frac{\sin (\tau|\nabla|)}{|\nabla|} \theta(i \nabla) G(\tau)\right\rangle d \tau d t \\
& +\int_{\mathbb{R}} \int_{\mathbb{R}}\left\langle\cos (t|\nabla|) \theta(i \nabla) F(t), \phi\left(\frac{\cdot-x_{0}}{R}\right) \cos (\tau|\nabla|) \theta(i \nabla) G(\tau)\right\rangle d \tau d t,
\end{aligned}
$$

where $\theta$ is defined in (2.6).

Proposition 5.3. We have

$$
\left|\left\langle A_{2}^{+}, \phi_{R} A_{2}^{-}\right\rangle+\left\langle B_{2}^{+}, \phi_{R} B_{2}^{-}\right\rangle\right| \lesssim R^{-\beta}
$$

for every $\beta<\frac{1}{26}$.

Proof. The proof proceeds as in the proof of Lemma 7.5 of [21]. We apply Lemma 5.4 with

$$
\begin{array}{r}
\tilde{F}(t)=\rho_{R}(t, x)\left(1-\sigma_{R}(t, x)\right)|\nabla|{ }^{s-1-\epsilon} P_{\leq N_{1}} F(u(t)), \\
G(\tau)=\rho_{R}(\tau, x)\left(1-\sigma_{R}(\tau, x)\right)|\nabla|^{s-1-\epsilon} P_{\leq N_{1}} F(u(\tau)) .
\end{array}
$$

Note that the hypotheses of the lemma imply that $F$ and $G$ have the required support. We therefore conclude

$$
\begin{aligned}
\left|\left\langle A_{2}^{+}, \phi_{R} A_{2}^{-}\right\rangle+\left\langle B_{2}^{+}, \phi_{R} B_{2}^{-}\right\rangle\right| & \lesssim R^{-1 / 26}\|\tilde{F}\|_{L_{t}^{\infty} L_{x}^{1}}\|G\|_{L_{t}^{\infty} L_{x}^{1}} \\
& \lesssim R^{-1 / 26}\|F(u)\|_{L_{t}^{\infty} L_{x}^{1}}^{2} \\
& \lesssim R^{-1 / 26}
\end{aligned}
$$

where we have used the Bernstein inequality along with the condition $s-1-\epsilon \geq 0$ to obtain the second inequality, and Proposition 3.1 to obtain the third inequality. Then $R \geq 1$ implies $R^{-1 / 26} \leq R^{-\beta}$ for every $\beta<\frac{1}{26}$, which gives the desired result, completing the proof of Proposition 5.3 . 
We are now ready to complete the proof of Lemma 5.1. In particular, collecting Proposition 5.1 Proposition 5.2 and Proposition 5.3, and invoking (5.8) we obtain

$$
\begin{aligned}
\left\|P_{\leq N_{1}}|\nabla|^{s-\epsilon} u(0)\right\|_{L_{x}^{2}\left(B_{R}\right)}+\left\|P_{\leq N_{1}}|\nabla|^{s-1-\epsilon} u_{t}(0)\right\|_{L_{x}^{2}\left(B_{R}\right)} \\
\quad \lesssim 2\left(R^{-2\left(\frac{1}{2}+\beta\right)}+2 R^{-\frac{1}{2}-\beta} R^{\frac{1}{2}}+R^{-\beta}\right) \\
\quad \lesssim R^{-\beta / 2}
\end{aligned}
$$

whenever $\epsilon$ and $\beta$ are sufficiently small, which concludes the proof of Lemma 5.1 .

Proof of Theorem 5.1. Iteratively apply Lemma 5.1, starting with $s=3 / 2$, to obtain $\left(u, u_{t}\right) \in L_{t}^{\infty}\left(\dot{H}_{x}^{s-\epsilon} \times \dot{H}_{x}^{s-\epsilon-1}\right)$, with $\epsilon$ sufficiently small to satisfy the hypotheses of Lemma 5.1, The claim then follows after finitely many iterations.

\section{Proof of Theorem 1.1}

In this section we conclude the proof of Theorem 1.1 by precluding each of the scenarios identified in Theorem 1.3. We begin with the finite time blow-up solution. As we mentioned in the introduction, the argument used to rule out this scenario in high dimensions $d \geq 6$ in 3 is also applicable to the present case. In particular, we outline the arguments used in the proof below.

Proposition 6.1 (Finite time blow-up solution). There is no solution $u: I \times \mathbb{R}^{5} \rightarrow$ $\mathbb{R}$ with maximal interval of existence $I$ satisfying the conditions of a finite time blow-up solution as in Theorem 1.3 .

Proof. We argue as in [3]; see also [4, 16, 21,22]. Without loss of generality (using the time translation and time reversal symmetries), suppose that $\sup I=1$. The first step is to show that there exists $x_{0} \in \mathbb{R}^{5}$ such that

$$
\operatorname{supp} u(t), \quad \operatorname{supp} u_{t}(t) \subset \overline{B\left(x_{0}, 1-t\right)}
$$

for all $t \in I$. This is accomplished by making use of the finite speed of propagation along with the definition of almost periodicity (for details, we refer the reader to the proof of Lemma 6.2 in 3 , and the references cited therein).

We now estimate the energy, using (6.1), Hölder's inequality and the Sobolev embedding to obtain

$$
\begin{aligned}
E\left(u(t), u_{t}(t)\right) & =\int_{\left|x-x_{0}\right| \leq 1-t} \frac{1}{2}|\nabla u(t)|^{2}+\frac{1}{2}\left|u_{t}(t)\right|^{2}+\frac{1}{4}|u(t)|^{4} d x \\
& \lesssim(1-t)\left[\|\nabla u\|_{L_{t}^{\infty} L_{x}^{5 / 2}}^{2}+\left\|u_{t}\right\|_{L_{t}^{\infty} L_{x}^{5 / 2}}^{2}+\|u\|_{L_{t}^{\infty} L_{x}^{5}}^{4}\right] \\
& \lesssim(1-t)\left\|\left(u, u_{t}\right)\right\|_{L_{t}^{\infty}\left(\dot{H}_{x}^{3 / 2} \times \dot{H}_{x}^{1 / 2}\right)}^{2} \\
& \lesssim(1-t),
\end{aligned}
$$

where we have used the a priori bound $\left(u, u_{t}\right) \in L_{t}^{\infty}\left(\dot{H}_{x}^{3 / 2} \times \dot{H}_{x}^{1 / 2}\right)$. Letting $t \rightarrow 1$ and using the conservation of energy, we obtain

$$
E\left(u(0), u_{t}(0)\right)=0,
$$

which gives $u \equiv 0$, contradicting $\|u\|_{L_{t, x}^{6}}=\infty$. 
We now turn to the two global scenarios identified in Theorem 1.3 the solitonlike solution and the low-to-high frequency cascade solution. We remark that the essential ingredient in precluding these scenarios is Theorem 5.1. We begin with the soliton-like solution.

Proposition 6.2 (Soliton-like solution). There is no solution $u: \mathbb{R} \times \mathbb{R}^{5} \rightarrow \mathbb{R}$ satisfying the conditions of a soliton-like solution as in Theorem 1.3 .

Proof. The proof proceeds as in 21; see also Section 8 of [3]. Suppose for contradiction that such a solution $u$ exists. Let $T>0$ be given. Invoking [22, Lemma 2.6] (see also Lemma 8.3 of [3]), we obtain

$$
\begin{aligned}
\int_{0}^{T} \int_{\mathbb{R}^{5}} \frac{|u(t, x)|^{4}}{|x|} d x d t & \geq \sum_{i=0}^{\lfloor T\rfloor-1} \int_{i}^{i+1} \int_{|x-x(t)| \leq R} \frac{|u(t, x)|^{4}}{|x|} d x d t \\
& \geq \sum_{i=0}^{\lfloor T\rfloor-1} \frac{1}{C^{\prime}+i} \int_{i}^{i+1} \int_{|x-x(t)| \leq R}|u(t, x)|^{4} d x d t \\
& \geq c \log \left(\frac{C^{\prime}+\lfloor T\rfloor}{C^{\prime}}\right) .
\end{aligned}
$$

On the other hand, the Morawetz estimate

$$
\int_{0}^{T} \int_{\mathbb{R}^{5}} \frac{|u(t, x)|^{4}}{|x|} d x d t \lesssim E\left(u_{0}, u_{1}\right)
$$

gives

$$
\begin{aligned}
& \log \left(\frac{C^{\prime}+\lfloor T\rfloor}{C^{\prime}}\right) \lesssim\left\|\left(u, u_{t}\right)\right\|_{L_{t}^{\infty}\left(\dot{H}_{x}^{1} \times L_{x}^{2}\right)}^{2}+\|u\|_{L_{t}^{\infty} L_{x}^{4}}^{4} \\
& \quad \lesssim\left\|\left(u, u_{t}\right)\right\|_{L_{t}^{\infty}\left(\dot{H}_{x}^{1} \times L_{x}^{2}\right)}^{2}+\|u\|_{L_{t}^{\infty} L_{x}^{5}}^{5 / 8}\|u\|_{L_{t}^{\infty} L_{x}^{3}}^{3 / 8} \\
& \quad \lesssim 1
\end{aligned}
$$

where we have used Theorem 5.1 and (3.1) to see the finiteness of the right hand side. Taking $T \rightarrow \infty$, we obtain a contradiction as desired.

To conclude, it therefore suffices to rule out the low-to-high frequency cascade solution of Theorem 1.3 For this, we first recall that for any almost periodic solution $u$ to (NLW), the compactness characterization (1.4) of almost periodicity implies that there exists $c(\eta)>0$ such that

$$
\left.\left.\int_{|\xi| \leq c(\eta) N(t)}|| \xi\right|^{3 / 2} \hat{u}(t, \xi)\right|^{2}+\left.\left.|| \xi\right|^{1 / 2} \hat{u}_{t}(t, \xi)\right|^{2} d \xi<\eta
$$

(see for instance, Remark 3.4 of [3] and (8.1) in [21]). We then have

Proposition 6.3 (Low-to-high frequency cascade solution). There is no solution $u: \mathbb{R} \times \mathbb{R}^{5} \rightarrow \mathbb{R}$ satisfying the conditions of a low-to-high frequency cascade solution as in Theorem 1.3 .

Proof. We proceed as in 21]; see also Section 9 of [3. Assuming for contradiction that such a $u$ existed, we choose a sequence $\left\{t_{n}\right\}$ such that $t_{n} \rightarrow \infty$ and $N\left(t_{n}\right) \rightarrow \infty$ as $n \rightarrow \infty$. Fix $\eta>0$, choose $c(\eta)$ as in (6.2), and fix a dyadic number $M \in\left(0, \frac{1}{2}\right)$. 
Suppose that $n$ is large enough to ensure $M<c(\eta) N\left(t_{n}\right)$. We may then write

$$
u_{\leq c(\eta) N\left(t_{n}\right)}\left(t_{n}\right)=u_{\leq M}\left(t_{n}\right)+u_{M<\cdot \leq c(\eta) N\left(t_{n}\right)}\left(t_{n}\right),
$$

so that by applying the Bernstein inequalities, Hölder's inequality, and (6.2), we obtain

$$
\begin{aligned}
\left\|\nabla u_{\leq c(\eta) N\left(t_{n}\right)}\left(t_{n}\right)\right\|_{L_{x}^{2}} \leq\left\|\nabla u_{\leq M}\left(t_{n}\right)\right\|_{L_{x}^{2}}+\left\|\nabla u_{M<\cdot \leq c(\eta) N\left(t_{n}\right)}\right\|_{L_{x}^{2}} \\
\quad \lesssim M^{\frac{5 \beta}{6}}\left\|\nabla u_{\leq M}\left(t_{n}\right)\right\|_{L_{x}^{3+\beta}}+M^{-1 / 2}\left\||\nabla|^{3 / 2} u_{\leq c(\eta) N\left(t_{n}\right)}\left(t_{n}\right)\right\|_{L_{x}^{2}} \\
\quad \lesssim M^{\frac{5 \beta}{6}}\left\|\langle x-x(t)\rangle^{\beta} \nabla u_{\leq 1}\right\|_{L_{t}^{\infty} L_{x}^{2}}\left\|\langle x-x(t)\rangle^{-\beta}\right\|_{L_{t}^{\infty} L_{x}^{6 / \beta}}+M^{-1 / 2} \eta \\
\quad \lesssim M^{\frac{5 \beta}{6}}+M^{-1 / 2} \eta,
\end{aligned}
$$

where we have invoked Theorem 5.1 and noted that

$$
\left\|\langle x-x(t)\rangle^{-\beta}\right\|_{L_{x}^{6 / \beta}}=\left(\int_{\mathbb{R}^{5}}\langle x-x(t)\rangle^{-6} d x\right)^{\beta / 6}<\infty
$$

for any $t \in \mathbb{R}$ to obtain the last inequality.

On the other hand, the Bernstein inequalities may also be applied to the high frequency portion of $u\left(t_{n}\right)$, giving

$$
\begin{aligned}
\left\|\nabla u_{>c(\eta) N\left(t_{n}\right)}\left(t_{n}\right)\right\|_{L_{x}^{2}} & \lesssim\left[c(\eta) N\left(t_{n}\right)\right]^{-1 / 2}\left\||\nabla|^{3 / 2} u_{>c(\eta) N\left(t_{n}\right)}\left(t_{n}\right)\right\|_{L_{x}^{2}} \\
& \lesssim\left[c(\eta) N\left(t_{n}\right)\right]^{-1 / 2}\left\||\nabla|^{3 / 2} u\right\|_{L_{t}^{\infty} L_{x}^{2}} \\
& \lesssim\left[c(\eta) N\left(t_{n}\right)\right]^{-1 / 2},
\end{aligned}
$$

where we have used the a priori bound $\left(u, u_{t}\right) \in L_{t}^{\infty}\left(\dot{H}_{x}^{3 / 2} \times \dot{H}_{x}^{1 / 2}\right)$. Combining (6.3) and (6.4) then gives the bound

$$
\left\|\nabla u\left(t_{n}\right)\right\|_{L_{x}^{2}} \leq M^{\frac{5 \beta}{6}}+M^{-1 / 2} \eta+\left[c(\eta) N\left(t_{n}\right)\right]^{-1 / 2} .
$$

Repeating these arguments for $u_{t}\left(t_{n}\right)$, we obtain

$$
\left\|P_{\leq c(\eta) N\left(t_{n}\right)} u_{t}\left(t_{n}\right)\right\|_{L_{x}^{2}} \lesssim M^{\frac{5 \beta}{6}}+M^{-1 / 2} \eta
$$

and

$$
\left\|P_{>c(\eta) N\left(t_{n}\right)} u_{t}\left(t_{n}\right)\right\|_{L_{x}^{2}} \lesssim\left[c(\eta) N\left(t_{n}\right)\right]^{-1 / 2},
$$

and we therefore obtain

$$
\left\|u_{t}\left(t_{n}\right)\right\|_{L_{x}^{2}} \leq M^{\frac{5 \beta}{6}}+M^{-1 / 2} \eta+\left[c(\eta) N\left(t_{n}\right)\right]^{-1 / 2}
$$

We now estimate the potential energy. For this, we note that the Sobolev embedding followed by interpolation gives

$$
\begin{aligned}
\left\|u\left(t_{n}\right)\right\|_{L_{x}^{4}} & \leq\left\||\nabla|^{5 / 4} u\left(t_{n}\right)\right\|_{L_{x}^{2}} \\
& \lesssim\left\|\nabla u\left(t_{n}\right)\right\|_{L_{x}^{2}}^{1 / 2}\left\||\nabla|^{3 / 2} u\left(t_{n}\right)\right\|_{L_{x}^{2}}^{1 / 2} \\
& \lesssim\left(M^{\frac{5 \beta}{6}}+M^{-1 / 2} \eta+\left[c(\eta) N\left(t_{n}\right)\right]^{-1 / 2}\right)^{1 / 2}
\end{aligned}
$$

where we have again used the a priori bound $\left(u, u_{t}\right) \in L_{t}^{\infty}\left(\dot{H}_{x}^{3 / 2} \times \dot{H}_{x}^{1 / 2}\right)$. 
Combining (6.5), 6.6) and (6.7), we obtain

$$
E\left(u_{0}, u_{1}\right)=E\left(u\left(t_{n}\right), u_{t}\left(t_{n}\right)\right) \lesssim \omega+\omega^{1 / 2},
$$

where

$$
\omega=M^{\frac{5 \beta}{6}}+M^{-1 / 2} \eta+\left[c(\eta) N\left(t_{n}\right)\right]^{-1 / 2} .
$$

Recall that (6.8) holds for all $n$ sufficiently large (depending on $M$ and $\eta$ ). Fixing $M$ and $\eta$, we now let $n \rightarrow \infty$ to obtain

$$
E\left(u_{0}, u_{1}\right) \lesssim M^{\frac{5 \beta}{6}}+M^{-1 / 2} \eta+\left(M^{\frac{5 \beta}{6}}+M^{-1 / 2} \eta\right)^{1 / 2}
$$

We now let $\eta \rightarrow 0$ followed by $M \rightarrow 0$ to conclude $E\left(u_{0}, u_{1}\right)=0$, and thus $u \equiv 0$, contradicting $\|u\|_{L_{t, x}^{6}}=\infty$.

\section{Appendix A. Proof of Lemma 5.4}

In this appendix, we prove the weak diffraction result, Lemma 5.4, which is used in the proof of Proposition 5.3

Proof. We argue as in Proposition 2.6 of 21 . To prove the lemma, we decompose the integral $I(F, G, R)$ into the sum of five terms, each of which will be estimated individually. Toward this end, we use the Plancherel theorem and the identity $\widehat{\phi\left(\frac{-x_{0}}{R}\right)}(\xi)=R^{5} e^{-i x_{0} \cdot \xi} \hat{\phi}(R \xi)$ to write

$$
\begin{aligned}
& \int\left\langle\nabla \frac{\sin (t|\nabla|)}{|\nabla|} \theta(i \nabla) F(t), \phi\left(\frac{\cdot-x_{0}}{R}\right) \nabla \frac{\sin (\tau|\nabla|)}{|\nabla|} \theta(i \nabla) G(\tau)\right\rangle d \tau d t
\end{aligned}
$$

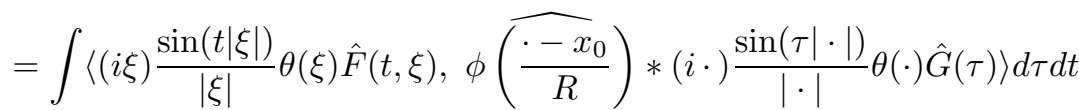

$$
\begin{aligned}
& =\int R^{5} \sin (t|\xi|) \sin (\tau|\eta|) \theta(\xi) \theta(\eta) \frac{\xi \cdot \eta}{|\xi||\eta|} \\
& \text {. } \overline{e^{-i x_{0} \cdot R(\xi-\eta)} \widehat{\phi}(R(\xi-\eta))} \hat{F}(t, \xi) \overline{\hat{G}(t, \eta)} d \eta d \xi d \tau d t .
\end{aligned}
$$

Summing this with a corresponding identity for the operator $\cos (t|\nabla|)$ and using the changes of variables $\xi \mapsto 2 \xi+\eta, \eta \mapsto \eta-\xi$, we find

$$
\begin{aligned}
& I(F, G, R)=\int R^{5}\left(\cos (t|\xi|-\tau|\eta|)-\sin (t|\xi|) \sin (\tau|\eta|)\left(1-\frac{\xi \cdot \eta}{|\xi||\eta|}\right)\right) \\
& \cdot \theta(\xi) \theta(\eta) \overline{e^{-i x_{0} \cdot R(\xi-\eta)} \widehat{\phi}(R(\xi-\eta))} \hat{F}(t, \xi) \overline{\hat{G}(\tau, \eta)} d \eta d \xi d \tau d t \\
& =\int \frac{R^{5}}{2}\left(\operatorname{Re}\left[e^{i(t|\xi|-\tau|\eta|)}\right]\left(1+\frac{\xi \cdot \eta}{|\xi||\eta|}\right)+\operatorname{Re}\left[e^{i(t|\xi|+\tau|\eta|)}\right]\left(1-\frac{\xi \cdot \eta}{|\xi||\eta|}\right)\right) \\
& \text {. } \theta(\xi) \theta(\eta) \overline{e^{-i x_{0} \cdot R(\xi-\eta)} \widehat{\phi}(R(\xi-\eta))} \hat{F}(t, \xi) \overline{\hat{G}(\tau, \eta)} d \eta d \xi d \tau d t \\
& =(I)+(I I)+(I I I)+(I V)+(V) \text {, }
\end{aligned}
$$


where we have set

$$
\begin{gathered}
(I)=\int 2^{4} R^{5} A(t, \tau, \mu, \nu) \phi\left(R^{11 / 25} \mu\right) \hat{F}(t, \mu+\nu) \overline{\hat{G}(\tau, \mu-\nu)} d \mu d \nu d \tau d t, \\
(I I)=\int 2^{4} R^{5} A_{1}(t, \tau, \mu, \nu)\left(1-\phi\left(R^{11 / 25} \mu\right)\right) \phi\left(R^{12 / 25} \nu\right) \\
\cdot \hat{F}(t, \mu+\nu) \overline{\hat{G}(\tau, \mu-\nu)} d \mu d \nu d \tau d t, \\
(I I I)=\int_{\left\{(t, \tau, \mu, \nu):|t+\tau| \leq R^{25 / 26}\right\}} 2^{4} R^{5} A_{2}(t, \tau, \mu, \nu)\left(1-\phi\left(R^{11 / 25} \mu\right)\right) \phi\left(R^{12 / 25} \nu\right) \\
\cdot \hat{F}(t, \mu+\nu) \overline{\hat{G}(\tau, \mu-\nu)} d \mu d \nu d \tau d t, \\
(I V)=\int_{\left\{(t, \tau, \mu, \nu):|t+\tau|>R^{25 / 26}\right\}} 2^{4} R^{5} A_{2}(t, \tau, \mu, \nu)\left(1-\phi\left(R^{11 / 25} \mu\right)\right) \phi\left(R^{12 / 25} \nu\right) \\
\cdot \hat{F}(t, \mu+\nu) \overline{\hat{G}(\tau, \mu-\nu)} d \mu d \nu d \tau d t, \\
(V)=\int_{2} 2^{4} R^{5} A(t, \tau, \mu, \nu)\left(1-\phi\left(R^{11 / 25} \mu\right)\right)\left(1-\phi\left(R^{12 / 25} \nu\right)\right) \\
\cdot \hat{F}(t, \mu+\nu) \overline{\hat{G}(\tau, \mu-\nu)} d \mu d \nu d \tau d t,
\end{gathered}
$$

and

$$
\begin{aligned}
& A(t, \tau, \mu, \nu):=A_{1}(t, \tau, \mu, \nu)+A_{2}(t, \tau, \mu, \nu), \\
& A_{1}(t, \tau, \mu, \nu):=\operatorname{Re}\left[e^{i(t|\mu+\nu|-\tau|\mu-\nu|)}\right]\left(1+\frac{(\mu+\nu) \cdot(\mu-\nu)}{|\mu+\nu||\mu-\nu|}\right) \\
& \cdot \theta(\mu+\nu) \theta(\mu-\nu) \overline{e^{-i x_{0} \cdot 2 R \nu} \widehat{\phi}(R(2 \nu))}, \\
& A_{2}(t, \tau, \mu, \nu):=\operatorname{Re}\left[e^{i(t|\mu+\nu|+\tau|\mu-\nu|)}\right]\left(1-\frac{(\mu+\nu) \cdot(\mu-\nu)}{|\mu+\nu||\mu-\nu|}\right) \\
& \cdot \theta(\mu+\nu) \theta(\mu-\nu) \overline{e^{-i x_{0} \cdot 2 R \nu} \widehat{\phi}(R(2 \nu))} .
\end{aligned}
$$

It now remains to estimate the terms $(I)-(V)$. For notational convenience, we set

$$
\begin{gathered}
X_{1}:=\left\{t: \operatorname{supp} F \cap\left(\{t\} \times \mathbb{R}^{5}\right) \neq \emptyset\right\} \times\left\{\tau: \operatorname{supp} G \cap\left(\{\tau\} \times \mathbb{R}^{5}\right) \neq \emptyset\right\}, \\
X:=X_{1} \times \mathbb{R}^{5} \times \mathbb{R}^{5},
\end{gathered}
$$

and note that the hypotheses of the lemma imply $\left|X_{1}\right| \leq\left(2 C_{1} R\right)^{2}$.

To estimate $(I)$, we use the change of variables $\mu \mapsto R^{-11 / 25} \mu$ and $\nu \mapsto R^{-1} \nu$ to obtain

$$
\begin{aligned}
|(I)| & \leq \int_{X} R^{5}\|F\|_{L_{t}^{\infty} L_{x}^{1}}\|G\|_{L_{t}^{\infty} L_{x}^{1}}|A(t, \tau, \mu, \nu)|\left|\phi\left(R^{11 / 25} \mu\right)\right| d \mu d \nu d \tau d t \\
& \leq \int_{X} R^{5}\|F\|_{L_{t}^{\infty} L_{x}^{1}}\|G\|_{L_{t}^{\infty} L_{x}^{1}}|\hat{\phi}(2 R \nu)|\left|\phi\left(R^{11 / 25} \mu\right)\right| d \mu d \nu d \tau d t \\
& =\int_{X} R^{-11 / 5}|\hat{\phi}(\nu)| \phi(\mu)\|F\|_{L_{t}^{\infty} L_{x}^{1}}\|G\|_{L_{t}^{\infty} L_{x}^{1}} d \mu d \nu d \tau d t \\
& \lesssim R^{-11 / 5}\left|X_{1}\right|\|F\|_{L_{t}^{\infty} L_{x}^{1}}\|G\|_{L_{t}^{\infty} L_{x}^{1}} \\
& \lesssim R^{-1 / 5}\|F\|_{L_{t}^{\infty} L_{x}^{1}}\|G\|_{L_{t}^{\infty} L_{x}^{1}}
\end{aligned}
$$


where to obtain the fourth line we have used $\phi \in C_{c}^{\infty}$ and the bound

$$
\int_{\mathbb{R}^{5}}|\hat{\phi}(\nu)| d \nu \leq C\left(\int_{|\nu| \leq 1} \frac{1}{|\nu|^{4}} d \nu+\int_{|\nu|>1} \frac{1}{|\nu|^{6}} d \nu\right)<\infty
$$

which follows from the observation that $\phi \in C_{c}^{\infty}$ allows us to choose constants $C_{m}>0$ such that

$$
|\hat{\phi}(\xi)| \leq C_{m}|\xi|^{-m}
$$

for every $m \geq 1$.

To estimate $(I I)$, we write $A_{1}(t, \tau, \mu, \nu)$ as the sum of two terms and estimate the resulting oscillatory integrals. More precisely, we obtain

$$
\begin{aligned}
|(I I)|= & \mid \int F(t, x) G(\tau, y)\left(\int 2^{4} R^{5} e^{i x \cdot(\mu+\nu)-i y \cdot(\mu-\nu)} A_{1}(t, \tau, \mu, \nu)\right. \\
& \left.\cdot\left(1-\phi\left(R^{11 / 25} \mu\right)\right) \phi\left(R^{12 / 25} \nu\right) d \mu d \nu\right) d x d y d \tau d t \mid \\
\lesssim & \left|\int F(t, x) G(\tau, y)\left(\int e^{i R \varphi_{1}(\mu, \nu)} \psi(\mu, \nu) d \mu d \nu\right) d x d y d \tau d t\right| \\
& +\left|\int F(t, x) G(\tau, y)\left(\int e^{i R \varphi_{-1}(\mu, \nu)} \psi(\mu, \nu) d \mu d \nu\right) d x d y d \tau d t\right| \\
\lesssim & \int_{\mathbb{R}^{5} \times \mathbb{R}^{5} \times X_{1}}|F(t, x) \| G(\tau, y)| R^{-74 / 25} d x d y d t d \tau \\
\lesssim & R^{-74 / 25}\left|X_{1}\right|\|F\|_{L_{t}^{\infty}} L_{x}^{1}\|G\|_{L_{t}^{\infty} L_{x}^{1}} \\
\lesssim & R^{-24 / 25}\|F\|_{L_{t}^{\infty} L_{x}^{1}}\|G\|_{L_{t}^{\infty} L_{x}^{1}}
\end{aligned}
$$

where we have used the oscillatory integral estimate

$$
\left|\int e^{i R \varphi_{\sigma}(\mu, \nu)} \psi(\mu, \nu) d \mu d \nu\right| \lesssim R^{-74 / 25}
$$

for $\sigma= \pm 1$, with

$$
\begin{aligned}
\varphi_{\sigma} & =\frac{1}{R}[\sigma(t|\mu+\nu|-\tau|\mu-\nu|)+\mu \cdot(x-y)+\nu \cdot(x+y)] \\
\psi & =\left(1-\phi\left(R^{11 / 25} \mu\right)\right) \\
& \frac{\phi\left(R^{12 / 25} \nu\right) R^{5}}{e^{-i x_{0} \cdot 2 R \nu} \hat{\phi}(2 R \nu)} \theta(\mu+\nu) \theta(\mu-\nu)\left(1+\frac{(\mu+\nu) \cdot(\mu-\nu)}{|\mu+\nu \| \mu-\nu|}\right) .
\end{aligned}
$$

To establish (A.4), let $(\mu, \nu, \tau, t)$ be a given point in the support of the integrand. We first show that $\left|\frac{\mu}{|\mu|} \cdot \nabla_{\mu} \varphi\right|$ is bounded away from zero. Indeed, using CauchySchwarz followed by the inequality $\sqrt{1-\frac{|\nu|^{2}}{|\mu+\nu|^{2}}} \leq \frac{\mu \cdot(\mu \pm \nu)}{|\mu| \mid \mu \pm \nu}$ (which follows from 
$|\mu| \geq R^{-11 / 25}$ and $\left.|\nu| \leq 2 R^{-12 / 25}\right)$, we obtain

$$
\begin{aligned}
\left|\frac{\mu}{|\mu|} \cdot \nabla_{\mu} \varphi\right| & =\left|\frac{t-\tau}{R}-\frac{t-\tau}{R}+\frac{\mu}{|\mu|} \cdot\left[\frac{\sigma t(\mu+\nu)}{R|\mu+\nu|}-\frac{\sigma \tau(\mu-\nu)}{R|\mu-\nu|}+\frac{(x-y)}{R}\right]\right| \\
& \geq C_{2}-C_{1} \max \left\{1-\frac{\mu \cdot(\mu+\nu)}{|\mu||\mu+\nu|}, 1-\frac{\mu \cdot(\mu-\nu)}{|\mu||\mu-\nu|}\right\} \\
& \geq C_{2}-C_{1}\left(1-\sqrt{1-\frac{|\nu|^{2}}{|\mu+\nu|^{2}}}\right) \\
& \geq C_{2}-\frac{C_{1}|\nu|^{2}}{|\mu+\nu|^{2}} \\
& \geq C_{2}-\frac{2 C_{1} R^{-24 / 25}}{\left(R^{-11 / 25}-2 R^{-12 / 25}\right)^{2}} \\
& \gtrsim 1,
\end{aligned}
$$

provided $R$ is chosen sufficiently large.

We now set $a=\left(\frac{\mu}{|\mu|} \cdot \nabla_{\mu} \varphi\right)^{-1} \frac{\mu}{|\mu|}$. Observing the identity $e^{i R \varphi}=R^{-6}\left(a \cdot i \nabla_{\mu}\right)^{6} e^{i R \varphi}$ and integrating by parts, we obtain

$$
\begin{aligned}
\left|\int e^{i R \varphi_{\sigma}(\mu, \nu)} \psi(\mu, \nu) d \mu d \nu\right| & \lesssim R^{-12 / 5} \sup _{|\nu| \leq 2 R^{-12 / 25}}\left|\int e^{i R \varphi} \psi d \mu\right| \\
& \lesssim R^{-42 / 5} \sup _{|\nu| \leq 2 R^{-12 / 25}} \int\left|\left(\nabla_{\mu} \cdot a\right)^{6} \psi\right| d \mu \\
& \lesssim R^{-42 / 5} \int_{|\mu| \geq R^{-11 / 25}} R^{5}|\mu|^{-6} d \mu \\
& \lesssim R^{-74 / 25},
\end{aligned}
$$

which establishes (A.4) as desired, where we have used the estimate

$$
\sup _{\nu}\left|\left(i \nabla_{\mu} \cdot a\right)^{6} \psi\right| \leq C R^{5}|\mu|^{-6} .
$$

We now turn to the estimate of $(I I I)$. To estimate this term, we begin by observing that for $R$ sufficiently large, the conditions $|\mu|>R^{-11 / 25}$ and $|\nu|<$ $2 R^{-12 / 25}$ give $|\mu|>2|\nu|$. We therefore obtain

$$
\begin{aligned}
|(I I I)| & \lesssim R^{5} \int_{\mathbb{R}^{5} \times \mathbb{R}^{5} \times S} \frac{|\nu|^{2}}{|\mu|^{2}} \theta(\mu+\nu)|\hat{\phi}(2 R \nu)|\|F\|_{L_{t}^{\infty} L_{x}^{1}}\|G\|_{L_{t}^{\infty} L_{x}^{1}} d \mu d \nu d \tau d t \\
& \lesssim R^{5}\|F\|_{L_{t}^{\infty} L_{x}^{1}}\|G\|_{L_{t}^{\infty} L_{x}^{1}} \int_{\mathbb{R}^{5} \times S}|\nu|^{2}|\hat{\phi}(2 R \nu)| d \nu d \tau d t \\
& \lesssim R^{-2}\|F\|_{L_{t}^{\infty} L_{x}^{1}}\|G\|_{L_{t}^{\infty} L_{x}^{1}}|S| \\
& \lesssim R^{-1 / 26}\|F\|_{L_{t}^{\infty} L_{x}^{1}}\|G\|_{L_{t}^{\infty} L_{x}^{1}}
\end{aligned}
$$

with

$$
S:=\left(X_{1} \cap\left\{(t, \tau):|t+\tau| \leq R^{25 / 26}\right\}\right),
$$

where for the first inequality we have used the bound

$$
1-\frac{(\mu+\nu) \cdot(\mu-\nu)}{|\mu+\nu||\mu-\nu|} \leq \frac{C|\nu|^{2}}{|\mu|^{2}}
$$


on the support of $\left(1-\phi\left(R^{11 / 25} \mu\right)\right) \phi\left(R^{12 / 25} \nu\right)$, while for the second and third inequalities we have used the bounds

$$
\int_{\mathbb{R}^{5}} \frac{\theta(\mu+\nu)}{|\mu|^{2}} d \mu=\int_{|\mu|<1}|\mu|^{-2} d \mu+\int_{|\mu| \geq 1} \theta(\mu+\nu) d \mu \leq C+\|\theta\|_{L^{1}}<\infty
$$

and

$$
\begin{aligned}
\int_{\mathbb{R}^{5}}|\nu|^{2}|\hat{\phi}(2 R \nu)| d \nu & =R^{-7} \int_{\mathbb{R}^{5}}|\nu|^{2}|\hat{\phi}(2 \nu)| d \nu \\
& \leq R^{-7}\left(\|\phi\|_{L^{1}} \int_{|\nu|<1}|\nu|^{2} d \nu+C \int_{|\nu| \geq 1}|2 \nu|^{-6} d \nu\right) \\
& \leq C R^{-7},
\end{aligned}
$$

respectively.

To estimate $(I V)$, we proceed similarly to our estimate of $(I I)$ above, writing $A_{2}(t, \tau, \mu, \nu)$ as the sum of two terms and estimating the resulting oscillatory integrals. More precisely, we obtain

$$
\begin{aligned}
|(I V)| \lesssim \mid \int F(t, x) G(\tau, y)\left(\int e^{i \widetilde{\varphi_{1}}(\mu, \nu)} \widetilde{\psi}(\mu, \nu)\right. \\
\left.\quad \cdot R^{5} \overline{e^{-i x_{0} \cdot 2 R \nu} \hat{\phi}(2 R \nu)} \phi\left(R^{12 / 25} \nu\right) d \mu d \nu\right) d x d y d \tau d t \mid \\
+\mid \int F(t, x) G(\tau, y)\left(\int e^{i \widetilde{\varphi_{-1}}(\mu, \nu)} \widetilde{\psi}(\mu, \nu)\right. \\
\left.\quad \cdot R^{5} \overline{e^{-i x_{0} \cdot 2 R \nu} \hat{\phi}(2 R \nu)} \phi\left(R^{12 / 25} \nu\right) d \mu d \nu\right) d x d y d \tau d t \mid \\
\lesssim \int_{\mathbb{R}^{5} \times \mathbb{R}^{5} \times X_{1}}|F(t, x) \| G(\tau, y)| R^{-129 / 52} d x d y d t d \tau \\
\lesssim\|F\|_{L_{t}^{\infty} L_{x}^{1}}\|G\|_{L_{t}^{\infty} L_{x}^{1}} R^{-129 / 52}\left|X_{1}\right| \\
\lesssim\|F\|_{L_{t}^{\infty} L_{x}^{1}}\|G\|_{L_{t}^{\infty} L_{x}^{1}} R^{-25 / 52},
\end{aligned}
$$

where we have used the oscillatory integral estimate

$$
\int\left(\int e^{i \widetilde{\varphi_{\sigma}}} \widetilde{\psi} d \mu\right) R^{5} \overline{e^{-i x_{0} \cdot 2 R \nu} \hat{\phi}(2 R \nu)} \phi\left(R^{12 / 25} \nu\right) d \nu \lesssim R^{-129 / 52}
$$

for $\sigma \in\{-1,1\}$ and

$$
\begin{aligned}
\widetilde{\varphi_{\sigma}} & =\sigma(t|\mu+\nu|+\tau|\mu-\nu|)+\mu(x-y)+\nu(x+y), \\
\widetilde{\psi} & =\theta(\mu+\nu) \theta(\mu-\nu)\left(1-\frac{(\mu+\nu) \cdot(\mu-\nu)}{|\mu+\nu||\mu-\nu|}\right)\left(1-\phi\left(R^{11 / 25} \mu\right)\right) .
\end{aligned}
$$

To obtain (A.6), we write the left hand side of (A.6) as

$$
\sum_{j=1}^{10} \int\left(\int e^{i \widetilde{\varphi}_{\sigma}} \widetilde{\psi} \eta_{j}(\mu) d \mu\right) R^{5} \overline{e^{-i x_{0} \cdot 2 R \nu} \hat{\phi}(2 R \nu)} \phi\left(R^{12 / 25} \nu\right) d \nu
$$

where the functions $\eta_{j}$ form a partition of unity subordinate to the open cover

for $k=1, \cdots, 5$.

$$
\left\{\max \left\{\left|\mu_{i}\right|: i \neq k\right\}<\frac{25}{12} \mu_{k}\right\}, \quad\left\{\max \left\{\left|\mu_{i}\right|: i \neq k\right\}<-\frac{25}{12} \mu_{k}\right\}
$$


We consider the $j=1$ term and note that by symmetry one may estimate the other terms in a similar manner. Invoking the Van der Corput lemma, we bound the modulus of the left hand side of (A.6) by

$$
\begin{aligned}
& \int R^{5}|\hat{\phi}(2 R \nu)|\left|\phi\left(R^{12 / 25} \nu\right)\right| \int\left(\int e^{i \widetilde{\varphi}_{\sigma}} \widetilde{\psi} \eta_{1} d \mu_{1}\right) d \mu_{2} \cdots d \mu_{5} \mid d \nu \\
& \lesssim \int_{\mathbb{R}^{5}} \int_{Y \times[0, \infty)}\left(\frac{\mu_{5}}{|t+\tau|}\right)^{1 / 2} R^{5}|\hat{\phi}(2 R \nu)|\left|\phi\left(R^{12 / 25} \nu\right)\right| \\
& \quad \cdot\left(\|\widetilde{\psi}\|_{L_{\mu_{1}}}+\left\|\partial_{\mu_{1}} \widetilde{\psi}\right\|_{L_{\mu_{1}}^{1}}\right) d \mu_{2} \cdots d \mu_{5} d \nu \\
& \lesssim \int_{\mathbb{R}^{5}} \int_{Y \times[0, \infty)}\left(\frac{|\nu|^{2}}{|t+\tau|^{1 / 2}}\right) R^{5}|\hat{\phi}(2 R \nu)|\left|\phi\left(R^{12 / 25} \nu\right)\right| \\
& \qquad \cdot\left(\frac{1}{\left\langle\mu_{5}\right\rangle^{8}}\left(2 \mu_{5}^{3 / 2}+\mu_{5}^{5 / 2}\right)\right) d \mu_{2} \cdots d \mu_{5} d \nu \\
& \lesssim R^{-129 / 52},
\end{aligned}
$$

with $Y=\left\{\left(\mu_{2}, \mu_{3}, \mu_{4}\right):\left|\mu_{i}\right| \leq \mu_{5}\right\}$, where to obtain the second inequality we have observed that for every $(\mu, \nu)$ in the support of the integrand and $(t, \tau) \in X_{1}$, we have the inequalities $|\mu|^{2}-\left|\mu_{1}\right|^{2} \sim|\mu|^{2}$ and $|\mu| \sim \mu_{5}$, as well as

$$
\left|\partial_{\mu_{1}}^{2} \widetilde{\varphi}_{\sigma}-\sigma \cdot(t+\tau) \frac{|\mu|^{2}-\mu_{1}^{2}}{|\mu|^{3}}\right| \leq \frac{C R^{1-1 / 25}}{|\mu|}
$$

and for $R$ sufficiently large,

$$
\begin{aligned}
\left|\partial_{\mu_{1}}^{2} \widetilde{\varphi}_{\sigma}\right| & \geq\left|(t+\tau) \frac{|\mu|^{2}-\mu_{1}^{2}}{|\mu|^{3}}\right|-\left|\partial_{\mu_{1}}^{2} \widetilde{\varphi}_{\sigma}-\sigma \cdot(t+\tau) \frac{|\mu|^{2}-\mu_{1}^{2}}{|\mu|^{3}}\right| \\
& \geq\left|(t+\tau) \frac{|\mu|^{2}-\mu_{1}^{2}}{|\mu|^{3}}\right|-\frac{C R^{1-1 / 25}}{|\mu|} \\
& \geq \frac{|t+\tau|}{\mu_{5}}-\frac{C R^{-1 / 650}|t+\tau|}{\mu_{5}} \\
& \geq \frac{|t+\tau|}{2 \mu_{5}},
\end{aligned}
$$

and to obtain the last inequality we have recalled that $\frac{1}{|t+\tau|^{1 / 2}} \leq \frac{1}{R^{25 / 52}}$ on the domain of integration.

It remains to estimate $(V)$, for which we proceed as in $(I)$. In particular, we observe that by (A.2), we have the inequality $|\hat{\phi}(2 R \nu)| \leq C|2 R \nu|^{-9}$, from which we 
obtain the bound

$$
\begin{aligned}
|(V)| & \lesssim \int_{X} R^{5}|A(t, \tau, \mu, \nu)|\left(1-\phi\left(R^{11 / 25} \mu\right)\right)\left(1-\phi\left(R^{12 / 25} \nu\right)\right) \\
& \lesssim\|F\|_{L_{t}^{\infty} L_{x}^{1}}\|G\|_{L_{t}^{\infty} L_{x}^{1}} d \mu d \nu d \tau d t \\
& \lesssim \int_{X} R^{5} \theta(\mu+\nu)|\hat{\phi}(2 R \nu)|\left(1-\phi\left(R^{12 / 25} \nu\right)\right)\|F\|_{L_{t}^{\infty} L_{x}^{1}}\|G\|_{L_{t}^{\infty} L_{x}^{1}} d \mu d \nu d \tau d t \\
& \lesssim\|\theta\|_{L^{1}}\|F\|_{L_{t}^{\infty} L_{x}^{1}}\|G\|_{L_{t}^{\infty} L_{x}^{1}} \int_{X \cap\left\{\nu>R^{-12 / 25}\right\}} R^{-4}|\nu|^{-9} d \nu d \tau d t \\
& \lesssim\|\theta\|_{L^{1}}\|F\|_{L_{t}^{\infty} L_{x}^{1}}\|G\|_{L_{t}^{\infty} L_{x}^{1}} R^{-52 / 25}\left|X_{1}\right| \\
& \lesssim\|F\|_{L_{t}^{\infty} L_{x}^{1}}\|G\|_{L_{t}^{\infty} L_{x}^{1}} R^{-2 / 25} .
\end{aligned}
$$

\section{ACKNOWLEDGEMENTS}

The author would like to thank M. Visan for bringing this problem to our attention and for comments on an earlier version of this manuscript, as well as W. Beckner and N. Pavlović for useful conversations. This material is based upon work supported by the National Science Foundation under agreement Nos. DMS-0635607 and DMS-0808042. Any opinions, findings and conclusions or recommendations expressed in this material are those of the author and do not necessarily reflect the views of the National Science Foundation.

\section{REFERENCES}

[1] Hajer Bahouri and Patrick Gérard, High frequency approximation of solutions to critical nonlinear wave equations, Amer. J. Math. 121 (1999), no. 1, 131-175. MR1705001(2000i:35123)

[2] Aynur Bulut, Maximizers for the Strichartz inequalities for the wave equation, Differential Integral Equations 23 (2010), no. 11-12, 1035-1072. MR2742477 (2011m:35202)

[3] Aynur Bulut, Global well-posedness and scattering for the defocusing energy-supercritical cubic nonlinear wave equation, J. Funct. Anal. 263 (2012), no. 6, 1609-1660, DOI 10.1016/j.jfa.2012.06.001. MR2948225

[4] Aynur Bulut, The radial defocusing energy-supercritical cubic nonlinear wave equation in $\mathbb{R}^{1+5}$, Nonlinearity 27 (2014), no. 8, 1859-1877, DOI 10.1088/0951-7715/27/8/1859. $\operatorname{MR} 3246158$

[5] Aynur Bulut, Global Well-posedness and Scattering for the Defocusing Energy-Supercritical Cubic Nonlinear Wave Equation, ProQuest LLC, Ann Arbor, MI, 2011. Thesis (Ph.D.)-The University of Texas at Austin. MR 3295410

[6] Aynur Bulut, The defocusing cubic nonlinear wave equation in the energy-supercritical regime, Recent advances in harmonic analysis and partial differential equations, Contemp. Math., vol. 581, Amer. Math. Soc., Providence, RI, 2012, pp. 1-11, DOI 10.1090/conm/581/11537. MR3013050

[7] James Colliander, Markus Keel, Gigliola Staffilani, Hideo Takaoka, and Terence Tao, Global well-posedness and scattering for the energy-critical nonlinear Schrödinger equation in $\mathbb{R}^{3}$, Ann. of Math. (2) 167 (2008), no. 3, 767-865, DOI 10.4007/annals.2008.167.767. MR 2415387 (2009f:35315)

[8] Luis Escauriaza, Gregory A. Seregin, and Vladimir Šverák, $L_{3, \infty}$-solutions of Navier-Stokes equations and backward uniqueness (Russian, with Russian summary), Uspekhi Mat. Nauk 58 (2003), no. 2(350), 3-44, DOI 10.1070/RM2003v058n02ABEH000609; English transl., Russian Math. Surveys 58 (2003), no. 2, 211-250. MR1992563 (2004m:35204)

[9] Jean Ginibre and Giorgio Velo, Generalized Strichartz inequalities for the wave equation, J. Funct. Anal. 133 (1995), no. 1, 50-68, DOI 10.1006/jfan.1995.1119. MR1351643(97a:46047) 
[10] Manoussos G. Grillakis, Regularity and asymptotic behaviour of the wave equation with a critical nonlinearity, Ann. of Math. (2) 132 (1990), no. 3, 485-509, DOI 10.2307/1971427. MR.1078267 (92c:35080)

[11] Manoussos G. Grillakis, Regularity for the wave equation with a critical nonlinearity, Comm. Pure Appl. Math. 45 (1992), no. 6, 749-774, DOI 10.1002/cpa.3160450604. MR.1162370 (93e:35073)

[12] Lev Kapitanskii, The Cauchy problem for the semilinear wave equation. I., II., III, Zap. Nauchn. Sem. Leningrad. Otdel. Mat. Inst. Steklov. (LOMI) 163 (1987), 76-104, 182 (1990), 38-85, and 181 (1990), 24-64. MR.918943 (89j:35088), MR.1064097(91i:35127) MR.1097579 (92d:35200)

[13] Markus Keel and Terence Tao, Endpoint Strichartz estimates, Amer. J. Math. 120 (1998), no. 5, 955-980. MR1646048(2000d:35018)

[14] Carlos E. Kenig and Frank Merle, Global well-posedness, scattering and blow-up for the energy-critical, focusing, non-linear Schrödinger equation in the radial case, Invent. Math. 166 (2006), no. 3, 645-675, DOI 10.1007/s00222-006-0011-4. MR2257393 (2007g:35232)

[15] Carlos E. Kenig and Frank Merle, Global well-posedness, scattering and blow-up for the energy-critical focusing non-linear wave equation, Acta Math. 201 (2008), no. 2, 147-212, DOI 10.1007/s11511-008-0031-6. MR2461508(2011a:35344)

[16] Carlos E. Kenig and Frank Merle, Nondispersive radial solutions to energy supercritical nonlinear wave equations, with applications, Amer. J. Math. 133 (2011), no. 4, 1029-1065, DOI 10.1353/ajm.2011.0029. MR2823870 (2012i:35244)

[17] Carlos E. Kenig and Frank Merle, Radial solutions to energy supercritical wave equations in odd dimensions, Discrete Contin. Dyn. Syst. 31 (2011), no. 4, 1365-1381, DOI 10.3934/dcds.2011.31.1365. MR2836357 (2012j:35256)

[18] Rowan Killip, Terence Tao, and Monica Visan, The cubic nonlinear Schrödinger equation in two dimensions with radial data, J. Eur. Math. Soc. (JEMS) 11 (2009), no. 6, 1203-1258, DOI 10.4171/JEMS/180. MR:2557134(2010m:35487)

[19] Rowan Killip and Monica Visan, The focusing energy-critical nonlinear Schrödinger equation in dimensions five and higher, Amer. J. Math. 132 (2010), no. 2, 361-424, DOI 10.1353/ajm.0.0107. MR2654778(2011e:35357)

[20] Rowan Killip and Monica Visan, Energy-supercritical NLS: critical $\dot{H}^{s}$-bounds imply scattering, Comm. Partial Differential Equations 35 (2010), no. 6, 945-987, DOI 10.1080/03605301003717084. MR2753625 (2011m:35357)

[21] Rowan Killip and Monica Visan, The defocusing energy-supercritical nonlinear wave equation in three space dimensions, Trans. Amer. Math. Soc. 363 (2011), no. 7, 3893-3934, DOI 10.1090/S0002-9947-2011-05400-0. MR2775831 (2012e:35161)

[22] Rowan Killip and Monica Visan, The radial defocusing energy-supercritical nonlinear wave equation in all space dimensions, Proc. Amer. Math. Soc. 139 (2011), no. 5, 1805-1817, DOI 10.1090/S0002-9939-2010-10615-9. MR2763767 (2011m:35229)

[23] Kenji Nakanishi, Scattering theory for the nonlinear Klein-Gordon equation with Sobolev critical power, Internat. Math. Res. Notices 1 (1999), 31-60, DOI 10.1155/S1073792899000021. MR.1666973 (2000a:35174)

[24] Hartmut Pecher, Nonlinear small data scattering for the wave and Klein-Gordon equation, Math. Z. 185 (1984), no. 2, 261-270, DOI 10.1007/BF01181697. MR731347 (85h:35165)

[25] Jeffrey Rauch, I. The $u^{5}$ Klein-Gordon equation. II. Anomalous singularities for semilinear wave equations, Collège de France Seminar, Vol. I (Paris, 1978/1979), Res. Notes in Math., vol. 53, Pitman, Boston, Mass., 1981, pp. 335-364. MR631403 (83a:35066)

[26] Jalal Shatah and Michael Struwe, Regularity results for nonlinear wave equations, Ann. of Math. (2) 138 (1993), no. 3, 503-518, DOI 10.2307/2946554. MR1247991 (95f:35164)

[27] Jalal Shatah and Michael Struwe, Well-posedness in the energy space for semilinear wave equations with critical growth, Internat. Math. Res. Notices 7 (1994), 303ff., approx. 7 pp. (electronic), DOI 10.1155/S1073792894000346. MR1283026(95e:35132)

[28] Jalal Shatah and Michael Struwe, Geometric wave equations, Courant Lecture Notes in Mathematics, vol. 2, New York University Courant Institute of Mathematical Sciences, New York, 1998. MR.1674843 (2000i:35135)

[29] Michael Struwe, Globally regular solutions to the $u^{5}$ Klein-Gordon equation, Ann. Scuola Norm. Sup. Pisa Cl. Sci. (4) 15 (1988), no. 3, 495-513 (1989). MR1015805 (90j:35142) 
[30] Terence Tao, Spacetime bounds for the energy-critical nonlinear wave equation in three spatial dimensions, Dyn. Partial Differ. Equ. 3 (2006), no. 2, 93-110. MR2227039 (2007c:35116)

[31] Terence Tao, Nonlinear dispersive equations, CBMS Regional Conference Series in Mathematics, vol. 106, Published for the Conference Board of the Mathematical Sciences, Washington, DC, 2006. Local and global analysis. MR2233925 (2008i:35211)

[32] Terence Tao, Monica Visan, and Xiaoyi Zhang, Minimal-mass blowup solutions of the masscritical NLS, Forum Math. 20 (2008), no. 5, 881-919, DOI 10.1515/FORUM.2008.042. MR:2445122(2009m:35495)

School of Mathematics, Institute for Advanced Study, Princeton, New Jersey 08540 E-mail address: abulut@math.ias.edu 